\title{
Adaptive through-thickness integration strategy for shell elements
}

Author: I.A. Burchitz

Date: February 2007

NIMR project: MC1.02121

Publication number: P07.1.016

Netherlands Institute for Metals Research 



\section{Summary}

Shell elements are commonly used in simulations of sheet metal forming using finite element analysis. Element matrices, due to their complexity, cannot conveniently be calculated in a closed form and therefore numerical integration is employed. The most commonly used rules for through-thickness integration in shell elements are Gauss quadrature and rules based on the Newton-Cotes formula.

Considering the integrand in the expression for the internal force vector, one may state that it is smooth only if during a finite element analysis the material remains in the elastic regime. When the material is in the elastic-plastic regime the integrand becomes non-smooth in, for example, out-of-plane direction since the stress profile may have a point of discontinuity at the surface that separates the elastic and plastic regions. The traditional numerical schemes do not perform well when integrating a non-smooth function and integration error may increase significantly. Therefore, if a shell element is in the elastic-plastic regime, the error due to numerical integration may be large and has to be dealt with.

A large number of sampling points in thickness direction may decrease the error due to numerical integration at the cost of increased computation time. Alternatively, the integration error can be decreased without a drastic increase in the computation time by using an adaptive through-thickness integration. A distinguishing feature of this integration scheme is that the location and/or the number of the sampling points is adapted to the through-thickness stress profile, leading to accurate numerical integration at minimal costs.

An adaptive through-thickness integration strategy for shell elements is developed in this report. The strategy consists of several algorithms that locate points of discontinuity in the out-of-plane stress profile, adapt sampling points, update values of internal variables and perform numerical integration.

Performance of the integration strategy is evaluated using a problem of bending of a beam under tension. It is shown that with adaptive integration it is possible to obtain accurate results with a very low number of sampling points. 



\section{Contents}

\begin{tabular}{ll}
\hline Summary & 3
\end{tabular}

\begin{tabular}{ll}
\hline Introduction & 7
\end{tabular}

1 Common integration rules for shell elements $\quad 9$

1.1 Newton-Cotes integration . . . . . . . . . . . . . . 10

1.1 .1 Trapezoidal rule . . . . . . . . . . . . . . . . . . 10

1.1.2 Error of Newton-Cotes integration. General formulae . . . . . . . . 12

1.1.3 Simpson's rule. . . . . . . . . . . . . . . . . . . . 13

1.2 Integration based on spline interpolation $\ldots \ldots \ldots$. . . . . . . . . 14

1.2.1 Derivation of a cubic spline . . . . . . . . . . . . . 14

1.2 .2 Spline integration . . . . . . . . . . . . . . . 17

1.3 Gauss integration . . . . . . . . . . . . . . . . . 18

1.4 Lobatto integration . . . . . . . . . . . . . . . . . . 22

1.5 Choice of an integration rule for shell elements . . . . . . . . . . . . . . . . 22

2 Origins of numerical integration error $\quad 25$

2.1 Analytical model of bending of a beam under tension . . . . . . . . . 25

2.1 .1 Strains and stresses . . . . . . . . . . . . . . . 25

2.1 .2 Stress resultants . . . . . . . . . . . . . . . . . . . . . 28

2.1.3 Change of curvature - springback . . . . . . . . . . . . . 30

2.1.4 Influence of in-plane tension on springback . . . . . . . . . . . . . 31

2.1.5 Results of analytical calculations . . . . . . . . . . . . . . 32

2.2 Numerical calculation of bending moment . . . . . . . . . . . . . . . 34

2.3 Error due to numerical integration . . . . . . . . . . . . . . . . . . 39 
3 Adaptive integration scheme for bending with tension problem 41

3.1 Rules for adaptive integration . . . . . . . . . . . . . . . . . . . . . . 41

3.2 Definition of adaptive noniterative strategy . . . . . . . . . . . . . . . . . . 43

3.2 .1 Interval manager . . . . . . . . . . . . . . . . . . . 43

3.2 .2 Interval processor . . . . . . . . . . . . . . . . . . . . . . 44

3.3 Calculation of bending moment using adaptive noniterative strategy . . . . 49

3.3 .1 Adaptive scheme $1 \ldots \ldots$. . . . . . . . . . . . . 50

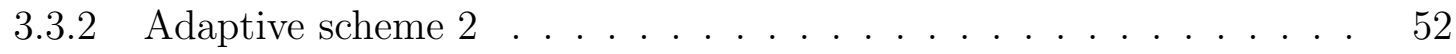

3.3 .3 Summary of results . . . . . . . . . . . . . . . . 54

4 Adaptive integration strategy for Kirchhoff triangular elements $\quad 55$

4.1 Overview of integration strategy . . . . . . . . . . . . . . . . . . 55

4.2 Discrete Kirchhoff triangular element . . . . . . . . . . . . . . . . . 56

4.3 Components of interval manager . . . . . . . . . . . . . . . . 58

4.3 .1 Calculation of points of discontinuity . . . . . . . . . . . . 59

4.3 .2 Update of internal variables . . . . . . . . . . . . . . . 66

4.4 Components of interval processor . . . . . . . . . . . . . . . . 68

5 Alternative numerical algorithm for locating points of discontinuity 69

5.1 Stress profiles in realistic forming conditions . . . . . . . . . . . . . . . . . 69

5.2 Numerical scheme to locate discontinuities . . . . . . . . . . . . . . . . 72

$\begin{array}{lr}\text { Conclusions } & 77\end{array}$

$\begin{array}{ll}\text { Acknowledgements } & 79\end{array}$

\begin{tabular}{ll}
\hline Appendix A & 81
\end{tabular}

$\begin{array}{ll}\text { Appendix B } & 83\end{array}$

$\begin{array}{lr}\text { Appendix C } & 85\end{array}$

$\begin{array}{lr}\text { Appendix D } & 87\end{array}$ 


\section{Introduction}

Accuracy of prediction of springback phenomenon in sheet metal forming is affected by factors that control quality of results of forming simulation. Various simplifications, introduced for making a simulation of forming more efficient, may have a significant influence on the accuracy of springback prediction. Assumptions in modelling material behaviour, method of unloading, chosen element type and mesh densities can be the reasons of deviation of the numerically predicted springback from that observed in real practice.

The material thickness in sheet metal forming is small comparing to the in-plane dimensions and therefore shell elements are often used in a finite element modelling of the process. The major advantage of shell elements is the reduced number of degrees of freedom of a finite element model, since the sheet geometry is described using only the midplane variables.

From the theory of finite element analysis it is known that the characteristic matrices of a shell element are volume integrals which cannot be easily calculated analytically. Therefore, it is a common practice to use the in-plane and the through-thickness numerical integration for calculating the element matrices. Numerical integration includes multiplication of an integrand's value at the predefined locations within an element by weight factors and adding results. Numerical schemes for the in-plane integration and the optimal location of integration points are discussed in $[1,2]$ and are not considered in this report.

To integrate through the thickness several integration rules are commonly used, namely, rules based on the Newton-Cotes integration formula, Gauss quadrature or Lobatto rule. Each of these integration rules has some advantages and the comparison of their performance in plate and shell elements can be found in [3]. The through-thickness stress profile defines the internal bending moment which governs a change of shape during an unloading. More than one integration point in thickness direction is needed to describe bending effects. For a material in the elastic regime the through-thickness stress profile is linear and the bending effects can be represented by a limited amount of the integration points (e.g. 2 for the Gauss quadrature). However, when the material undergoes plastic deformations there appear points of discontinuity in the stress distribution. At the presence of the discontinuity points none of the integration rules is capable of calculating accurately and the number of the integration points required to represent the nonlinear stress profile increases [4]. Wagoner et al. [5] showed that depending on the material, process parameters and the integration rule $5-50$ integration points may be needed in the thickness direction to minimise the influence of numerical integration on the springback prediction. Such a broad range for the adequate number of the integration points shows inefficiency 
of commonly used integration rules. Furthermore, using more than 20 integration points places high demands on computational costs and is very undesirable.

To overcome this problem one may use the approach proposed by Simo et al. [6]. In this approach a constitutive model is formulated directly in terms of stress resultants which allows eliminating entirely the through-thickness integration from a finite element computational procedure. However, as mentioned by the authors themselves, the main disadvantage of this approach is that even the simplest yield criteria for the elastic-plastic analysis take a considerably more complex functional form when expressed in stress resultants. As a result, implementation of three-dimensional material models becomes a difficult task.

The second approach is to use an adaptive integration, which identifies presence of the points of discontinuity and adapts the integration rule depending on the stress profile. An adaptive integration strategy is developed in this report. To be used in the finite element analysis the adaptive strategy has to include several algorithms:

- algorithm to locate the points of discontinuity in the through-thickness stress profile for an arbitrary history of deformation;

- algorithm to manage the location and the number of integration points and to perform the actual integration;

- algorithm to update internal variables at newly introduced or relocated integration points.

Defining the points of discontinuity in the stress profile in case of an arbitrary loading is not a trivial task. Multiple points of discontinuity may be present and their position and the number depends on the deformation path. Some attempts have been made to trace the stress profile discontinuities (see [7]), however, due to the underlying assumptions the presented algorithms are only applicable to a limited number of deformation scenarios, namely bending with tension and bending/unbending under tension. In light of that, it is decided to split the development of the adaptive integration strategy into two phases. During the first phase the existing algorithms for locating the points of discontinuity will be tested and a simplified adaptive strategy will be developed. During the second phase the strategy will be extended to make it applicable to any loading scenario and to make it capable of dealing with an arbitrary number of the points of discontinuity.

This report focuses on the first phase and describes the development of the simplified adaptive strategy for shell elements. It is started with a brief introduction to available integration strategies for plate and shell elements and outlines their advantages and disadvantages. Origins of the numerical integration error are discussed in chapter 2 . In chapter 3 an adaptive integration strategy is developed for the problem of bending of a beam under tension. Major components of the strategy are described and its potential is shown. The adaptive integration strategy is elaborated further in chapter 4 to be applicable in a finite element analysis with shell elements. A simple numerical algorithm for locating the points of discontinuity which occur in the stress profile during pure elastic-plastic bending is presented in chapter 5. It can be used as a basis for developing alternative numerical algorithms for locating the points of discontinuity in case of an arbitrary loading. The report is finished with conclusions and a brief description of the future work. 


\section{Chapter 1}

\section{Common integration rules for shell elements}

In finite element analysis the element characteristic matrices are often complex volume integrals that are usually solved using numerical integration. Formulation of shell elements requires that the characteristic matrices are found by integrating numerically in-plane and in thickness direction. For a triangular shell element there are usually three in-plane sampling points that are uniformly distributed over the triangle. Their location is symmetric in terms of the area coordinates. For the integration in thickness direction there are several widely used numerical procedures, namely trapezoidal, Simpson's, Lobatto rules and Gauss quadrature.

The global idea behind numerical integration is presented here. Let $I(f)=\int_{a}^{b} f(x) d x$ be an integral to evaluate. For the integrand $f(x)$ one can find an approximating family $\left\{f_{n}(x) \mid n \geq 1\right\}$ and define:

$$
I_{n}(f)=\int_{a}^{b} f_{n}(x) d x=I\left(f_{n}\right)
$$

It is required that the approximations $f_{n}(x)$ satisfy:

$$
\left\|f-f_{n}\right\|_{\infty} \rightarrow 0 \text { with } n \rightarrow \infty
$$

An additional requirement is that the form of $f_{n}(x)$ must be chosen such that $I_{n}(f)$ can be evaluated easily. The error of the integration can be defined as follows:

$$
E_{n}(f)=I(f)-I_{n}(f)=\int_{a}^{b}\left[f(x)-f_{n}(x)\right] d x
$$

Finally, most numerical integrals after the evaluation will have the following form:

$$
I_{n}(f)=\sum_{j=1}^{n} w_{j, n} f\left(x_{j, n}\right) \mid n \geq 1
$$

The coefficients $w_{j, n}$ are the integration weights and $x_{j, n}$ define the location of sampling points that usually belong to the interval $[a, b]$. The dependence of the weights and the locations of the sampling points on $n$ in the following text will be understood implicitly. Thus, to simplify, expressions $w_{j, n}$ and $x_{j, n}$ will be written as $w_{j}$ and $x_{j}$. 


\subsection{Newton-Cotes integration}

Let $I(f)=\int_{a}^{b} f(x) d x$ be an integral to be evaluated numerically and $[a, b]$ is the interval of integration. For $n \geq 1$ one can define $h=(b-a) / n$ and the location of any sampling point within the integration interval can be found from $x_{j}=a+j h$ for $j=0,1, \ldots, n$. To define $I_{n}(f)$ the integrand $f(x)$ should be replaced with its interpolating polynomial $p_{n}(x)$ on the sampling points $x_{0}, x_{1}, \ldots, x_{n}$ :

$$
I(f)=\int_{a}^{b} f(x) d x \doteq I_{n}(f)=\int_{a}^{b} p_{n}(x) d x
$$

$p_{n}(x)$ can be found using the Lagrange's formula for the interpolating polynomial which in general form is written as follows:

$$
p_{n}(x)=\sum_{j=0}^{n} l_{j}(x) f\left(x_{j}\right), \text { where } l_{j}(x)=\prod_{i \neq j}^{n}\left(\frac{x-x_{i}}{x_{j}-x_{i}}\right), \quad \text { for } j=0,1, \ldots, n
$$

Therefore $I_{n}(f)$ becomes:

$$
I_{n}(f)=\int_{a}^{b} \sum_{j=0}^{n} l_{j}(x) f\left(x_{j}\right) d x=\sum_{j=0}^{n} w_{j} f\left(x_{j}\right)
$$

where $\int_{a}^{b} l_{j}(x) d x=w_{j}$, for $j=0,1, \ldots, n$.

The Newton-Cotes formula provides a general framework for deriving several well-known integration rules. For example, with $n=1$ it leads to trapezoidal rule and with $n=2$ to Simpson's rule.

\subsubsection{Trapezoidal rule}

The trapezoidal rule is based on approximating $f(x)$ on $[a, b]$ by the first order polynomial or a straight line, as shown in figure 1.1. Using the Langrange's formula for the interpolating polynomial (equation 1.6) one can define the approximating polynomial $p_{1}(x)$ :

$$
p_{1}(x)=\frac{x-x_{1}}{x_{0}-x_{1}} f\left(x_{0}\right)+\frac{x-x_{0}}{x_{1}-x_{0}} f\left(x_{1}\right)=\frac{x-b}{a-b} f(a)+\frac{x-a}{b-a} f(b)
$$

and

$$
I_{1}(f)=\int_{a}^{b}\left(\frac{x-b}{a-b} f(a)+\frac{x-a}{b-a} f(b)\right) d x=\frac{b-a}{2}(f(a)+f(b))
$$

Prior to deriving a formula for the error of integration with the trapezoidal rule some mathematical concepts are introduced. For a given function $f(x)$ one can define the first- and second-order divided differences of this function, see equations 1.10 and 1.11 respectively:

$$
f\left[x_{0}, x_{1}\right]=\frac{f\left(x_{1}\right)-f\left(x_{0}\right)}{x_{1}-x_{0}}
$$




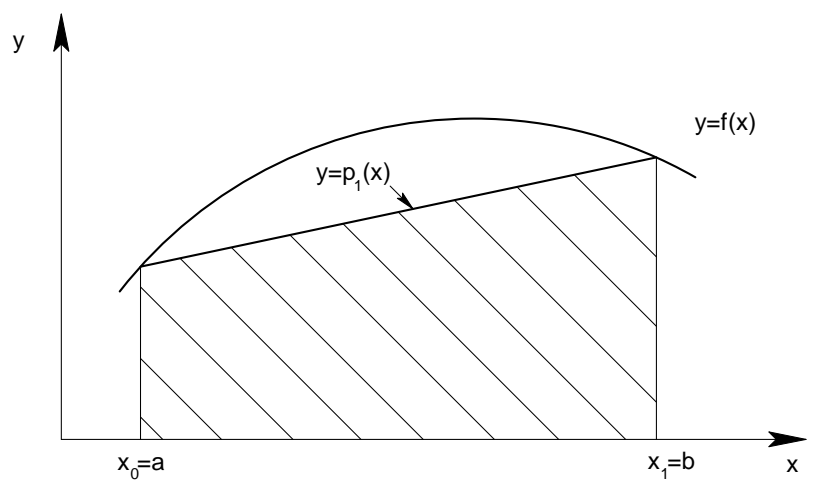

Figure 1.1: Integration with trapezoidal rule.

$$
f\left[x_{0}, x_{1}, x_{2}\right]=\frac{f\left[x_{1}, x_{2}\right]-f\left[x_{0}, x_{1}\right]}{x_{2}-x_{0}}
$$

The first- and second-order divided differences are related to the derivatives of $f(x)[8]$ :

$$
f\left[x_{0}, x_{1}\right]=f^{\prime}(\xi) \text { and } \quad f\left[x_{0}, x_{1}, x_{2}\right]=\frac{1}{2} f^{\prime \prime}(\zeta)
$$

Where $\xi$ is a value between $x_{0}$ and $x_{1}$ and $\zeta$ is a value between the maximum and minimum of $x_{0}, x_{1}$ and $x_{2}$.

Theorem 1. Integral mean value: Let $f(x)$ be a continuous function on $[a, b]$ and let $w(x)$ be a function which is nonnegative and integrable on $[a, b]$, then:

$$
\int_{a}^{b} w(x) f(x) d x=f(\xi) \int_{a}^{b} w(x) d x
$$

for some $\xi$ on $[a, b]$. The proof of this theorem is given in [8].

Using the introduced notation and equation 1.3 the integration error formula can be derived. Assuming that the function $f(x)$ is twice continuously differentiable on $[a, b]$ :

$$
\begin{aligned}
E_{1}(f) & =\int_{a}^{b}\left[f(x)-p_{1}(x)\right] d x=\int_{a}^{b}\left[f(x)-\frac{x-b}{a-b} f(a)-\frac{x-a}{b-a} f(b)\right] d x= \\
& =\int_{a}^{b}(x-a)(x-b) f[a, b, x] d x
\end{aligned}
$$

Applying the integral mean value theorem:

$$
\begin{aligned}
E_{1}(f) & =f[a, b, \xi] \int_{a}^{b}(x-a)(x-b) d x, \quad \text { for some } \quad a \leq \xi \leq b \\
E_{1}(f) & =\frac{1}{2} f^{\prime \prime}(\eta) \int_{a}^{b}\left(x^{2}-(a+b) x+a b\right) d x=\frac{1}{2} f^{\prime \prime}(\eta)\left(\left.\frac{x^{3}}{3}\right|_{a} ^{b}-\left.(a+b) \frac{x^{2}}{2}\right|_{a} ^{b}+\left.a b x\right|_{a} ^{b}\right)= \\
& =\frac{1}{2} f^{\prime \prime}(\eta)\left(-\frac{1}{6}(b-a)^{3}\right)=-\frac{(b-a)^{3}}{12} f^{\prime \prime}(\eta), \quad \text { for some } \quad \eta \in[a, b]
\end{aligned}
$$


Equation 1.15 shows that if the interval $(b-a)$ is not sufficiently small the error of integration with the trapezoidal rule will be high. In practice the integration interval $[a, b]$ is divided into $n$ smaller subintervals of equal size and the integral $I(f)$ is broken into a sum of integrals over every subinterval. If $h=(b-a) / n \mid n \geq 1$, then $x_{j}=a+j h$ for $j=0,1, \ldots, n$ and

$$
I(f)=\int_{a}^{b} f(x) d x=\sum_{j=1}^{n} \int_{x_{j-1}}^{x_{j}} f(x) d x \doteq \sum_{j=1}^{n} \frac{h}{2}\left(f\left(x_{j-1}\right)+f\left(x_{j}\right)\right)
$$

Expanding this sum and adding the first an the last terms one obtains the formula of the composite trapezoidal rule:

$$
I_{n}(f)=h\left(\frac{1}{2} f\left(x_{0}\right)+f\left(x_{1}\right)+f\left(x_{2}\right)+\cdots+f\left(x_{n-1}\right)+\frac{1}{2} f\left(x_{n}\right)\right)
$$

The error formula becomes [8]:

$$
E_{n}(f)=-\frac{(b-a) h^{2}}{12} f^{\prime \prime}(\eta), \quad \text { with } \quad \eta \in[a, b]
$$

Equation 1.18 shows that when $n$ is doubled and consequently $h$ is halved, the error decreases by a factor of 4 . The factor is the speed of convergence of the trapezoidal rule.

\subsubsection{Error of Newton-Cotes integration. General formulae}

The general expression for the error of numerical integration using the Newton-Cotes formulae is defined by the following theorem [8].

Theorem 2. For $n$ even and assuming that $f(x)$ is $n+2$ times continuously differentiable on $[a, b]$

$$
E_{n}(f)=C_{n} h^{n+3} f^{(n+2)}(\eta), \quad \text { for some } \quad \eta \in[a, b]
$$

and

$$
C_{n}=\frac{1}{(n+2) !} \int_{0}^{n} \mu^{2}(\mu-1) \cdots(\mu-n) d \mu, \quad \text { with } \quad 0 \leq \mu \leq n
$$

For $\mathrm{n}$ odd and assuming that $f(x)$ is $n+1$ times continuously differentiable on $[a, b]$

$$
E_{n}(f)=C_{n} h^{n+2} f^{(n+1)}(\eta)
$$

and

$$
C_{n}=\frac{1}{(n+1) !} \int_{0}^{n} \mu(\mu-1) \cdots(\mu-n) d \mu
$$

The proof of this theorem is not provided here. It is similar to the derivations of the error formula for the trapezoidal integration and can be found in [8]. 


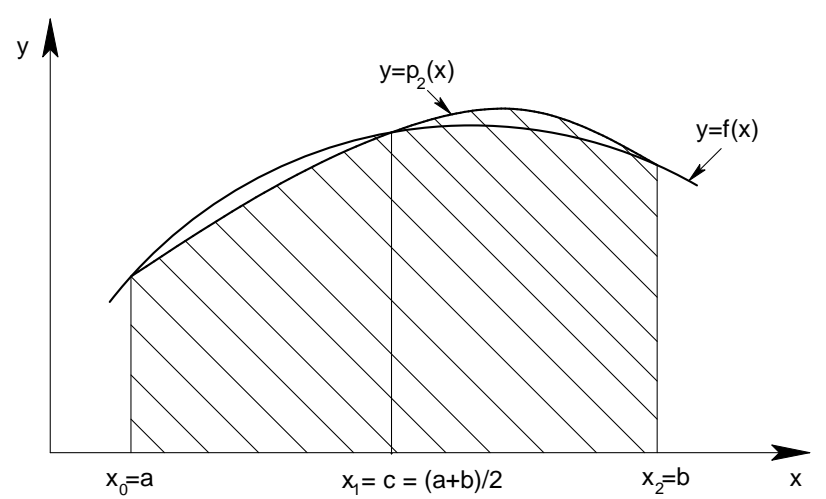

Figure 1.2: Integration with Simpson's rule.

\subsubsection{Simpson's rule}

Simpson's rule can be derived by using a quadratic interpolating polynomial $p_{2}(x)$ to approximate $f(x)$ on the interval $[a, b]$ (see figure 1.2). The approximating function $p_{2}(x)$ can be derived using the Lagrange's formula for the interpolating polynomial. From equation 1.6:

$$
\begin{aligned}
p_{2}(x) & =\frac{\left(x-x_{2}\right)\left(x-x_{1}\right)}{\left(x_{0}-x_{2}\right)\left(x_{0}-x_{1}\right)} f\left(x_{0}\right)+\frac{\left(x-x_{0}\right)\left(x-x_{2}\right)}{\left(x_{1}-x_{0}\right)\left(x_{1}-x_{2}\right)} f\left(x_{1}\right)+\frac{\left(x-x_{0}\right)\left(x-x_{1}\right)}{\left(x_{2}-x_{0}\right)\left(x_{2}-x_{1}\right)} f\left(x_{2}\right)= \\
& =\frac{(x-b)(x-c)}{(a-b)(a-c)} f(a)+\frac{(x-a)(x-b)}{(c-a)(c-b)} f(c)+\frac{(x-a)(x-c)}{(b-a)(b-c)} f(b)
\end{aligned}
$$

Therefore

$$
I_{2}(f)=\int_{a}^{b}\left(\frac{(x-b)(x-c)}{(a-b)(a-c)} f(a)+\frac{(x-a)(x-b)}{(c-a)(c-b)} f(c)+\frac{(x-a)(x-c)}{(b-a)(b-c)} f(b)\right) d x
$$

Performing integration one obtains:

$$
I_{2}(f)=\frac{h}{3}(f(a)+4 f(c)+f(b)), \quad h=\frac{b-a}{2}
$$

Equations 1.19 and 1.20 can be used to obtain the error formula for Simpson's integration:

$$
E_{2}(f)=-\frac{h^{5}}{90} f^{(4)}(\eta), \quad \text { where } \quad \eta \in[a, b]
$$

Splitting the interval $[a, b]$ into $n$ even and equal subintervals gives:

$$
I(f)=\int_{a}^{b} f(x) d x=\sum_{j=1}^{n / 2} \int_{x_{2 j-2}}^{x_{2 j}} f(x) d x=\sum_{j=1}^{n / 2} \frac{h}{3}\left(f\left(x_{2 j-2}\right)+4 f\left(x_{2 j-1}\right)+f\left(x_{2 j}\right)\right)
$$

Expanding this equation and gathering terms gives the composite Simpson's rule:

$$
I_{n}(f)=\frac{h}{3}\left(f\left(x_{0}\right)+4 f\left(x_{1}\right)+2 f\left(x_{2}\right)+4 f\left(x_{3}\right)+\cdots+2 f\left(x_{n-2}\right)+4 f\left(x_{n-1}\right)+f\left(x_{n}\right)\right)
$$


The error of integration with the composite Simpson's rule [8]:

$$
E_{n}(f)=-\frac{h^{4}(b-a)}{180} f^{(4)}(\eta), \quad \text { with } \quad \eta \in[a, b]
$$

Equation 1.29 shows that the error of integration with the Simpson's rule is proportional to $h^{4}$, which becomes a factor of 16 when $h$ is halved. It is clear that the Simpson's rule is superior to the trapezoidal rule since the speed of convergence is higher.

Other integration rules can be derived by using the Newton-Cotes formula 1.7 and higher order polynomials to approximate $f(x)$. However, it is usually recommended not to use higher order formulae since they may not converge for well-behaved integrands [8].

\subsection{Integration based on spline interpolation}

Splines are known as functions which can be used to put a very smooth curve through a set of points. They do not show the oscillatory behaviour which is a characteristic of high order polynomials and are good candidates for approximation of the integrand.

To define a spline let the interval $[a, b]$ be divided into $n$ subintervals $a<x_{0}<x_{1}<\cdots<$ $x_{n-1}<x_{n}<b$ that are not necessarily of equal length. A spline $S(x)$ of degree $m$ can be called a function defined on $[a, b]$ which $[9]$ :

- coincides with a polynomial of degree $m$ on each subinterval: $\left[x_{i-1}, x_{i}\right], i=1,2, \ldots, n$;

- is $m-1$ times continuously differentiable.

A cubic spline will be considered in the following text. The abscissas $x_{i}$ are called nodes of the spline and it is assumed that a spline $S(x)$ interpolates a set of points $\left(x_{0}, y_{0}\right), \ldots,\left(x_{n}, y_{n}\right)$ if $S\left(x_{i}\right)=y_{i}$ for $i=0,1, \ldots, n$.

\subsubsection{Derivation of a cubic spline}

To derive a mathematical representation for the cubic spline $S(x)$ the following procedure can be followed $[9,10]$. Let $h_{j}=x_{j+1}-x_{j}$ for $j=0,1, \ldots, n-1$. Since $S(x)$ is piecewise cubic then $S^{\prime}(x)$ is piecewise quadratic and $S^{\prime \prime}(x)$ is piecewise linear and continuous. Therefore, using the Lagrange formula for the interpolating polynomial (equation 1.6) one can write:

$$
\begin{array}{r}
S^{\prime \prime}(x)=\frac{x-x_{j+1}}{x_{j}-x_{j+1}} M_{j}+\frac{x-x_{j}}{x_{j+1}-x_{j}} M_{j+1}=\frac{x_{j+1}-x}{h_{j}} M_{j}+\frac{x-x_{j}}{h_{j}} M_{j+1}, \\
\text { on } \Delta_{j}=\left[x_{j}, x_{j+1}\right]
\end{array}
$$

where

$$
M_{j}=S^{\prime \prime}\left(x_{j}\right) \text { and } \quad M_{j+1}=S^{\prime \prime}\left(x_{j+1}\right)
$$


Performing twice the indefinite integration on 1.30 it is possible to obtain:

$$
S(x)=\frac{\left(x_{j+1}-x\right)^{3}}{6 h_{j}} M_{j}+\frac{\left(x-x_{j}\right)^{3}}{6 h_{j}} M_{j+1}+\left(x_{j+1}-x\right) A_{j}+\left(x-x_{j}\right) B_{j}, \text { on } \Delta_{j}
$$

Using $S\left(x_{j}\right)=y_{j}$ and $S\left(x_{j+1}\right)=y_{j+1}$ it is possible to determine $A_{j}$ and $B_{j}$ :

$$
\begin{aligned}
y_{j} & =\frac{\left(x_{j+1}-x_{j}\right)^{3}}{6 h_{j}} M_{j}+\left(x_{j+1}-x_{j}\right) A_{j} \Rightarrow \\
y_{j} & =\frac{h_{j}^{2}}{6} M_{j}+h_{j} A_{j} \Rightarrow \\
A_{j} & =\frac{6 y_{j}-h_{j}^{2} M_{j}}{6 h_{j}}
\end{aligned}
$$

and

$$
\begin{aligned}
y_{j+1} & =\frac{\left(x_{j+1}-x_{j}\right)^{3}}{6 h_{j}} M_{j+1}+\left(x_{j+1}-x_{j}\right) B_{j} \Rightarrow \\
y_{j+1} & =\frac{h_{j}^{2}}{6} M_{j+1}+h_{j} B_{j} \Rightarrow \\
B_{j} & =\frac{6 y_{j+1}-h_{j}^{2} M_{j+1}}{6 h_{j}}
\end{aligned}
$$

Therefore the equation for the cubic spline $S(x)$ becomes:

$$
\begin{aligned}
S(x) & =\frac{\left(x_{j+1}-x\right)^{3}}{6 h_{j}} M_{j}+\frac{\left(x-x_{j}\right)^{3}}{6 h_{j}} M_{j+1}+\left(y_{j}-\frac{h_{j}^{2} M_{j}}{6}\right) \frac{\left(x_{j+1}-x\right)}{h_{j}}+ \\
& +\left(y_{j+1}-\frac{h_{j}^{2} M_{j+1}}{6}\right) \frac{\left(x-x_{j}\right)}{h_{j}}
\end{aligned}
$$

Using equation 1.34 it is possible to derive an alternative formula for $S(x)$ which is more convenient for plotting the cubic spline on every subinterval $\left[x_{j}, x_{j+1}\right]$. The derivation can be found in Appendix A.

$M_{j}$ and $M_{j+1}$ are unknown values and to find expressions for these unknowns one can differentiate equation 1.34 to get:

$$
S^{\prime}(x)=-\frac{\left(x_{j+1}-x\right)^{2}}{2 h_{j}} M_{j}+\frac{\left(x-x_{j}\right)^{2}}{2 h_{j}} M_{j+1}+\frac{y_{j+1}-y_{j}}{h_{j}}-\frac{M_{j+1}-M_{j}}{6} h_{j}, \quad \text { on } \quad \Delta_{j}
$$

Noting the special values:

$$
\begin{aligned}
& S^{\prime}\left(x_{j}^{-}\right)=\frac{h_{j-1} M_{j}}{3}+\frac{h_{j-1} M_{j-1}}{6}+\frac{y_{j}-y_{j-1}}{h_{j-1}} \\
& S^{\prime}\left(x_{j}^{+}\right)=-\frac{h_{j} M_{j}}{3}-\frac{h_{j} M_{j+1}}{6}+\frac{y_{j+1}-y_{j}}{h_{j}}
\end{aligned}
$$


and recalling the fact that $S^{\prime}(x)$ is required to be continuous, which means that $S^{\prime}\left(x_{j}^{-}\right)=$ $S^{\prime}\left(x_{j}^{+}\right)$, one obtains:

$$
\begin{aligned}
\frac{h_{j-1} M_{j}}{3}+\frac{h_{j-1} M_{j-1}}{6}+\frac{y_{j}-y_{j-1}}{h_{j-1}}= & -\frac{h_{j} M_{j}}{3}-\frac{h_{j} M_{j+1}}{6}+\frac{y_{j+1}-y_{j}}{h_{j}} \Rightarrow \\
\frac{h_{j-1} M_{j-1}}{6}+\frac{\left(h_{j-1}+h_{j}\right) M_{j}}{3}+\frac{h_{j} M_{j+1}}{6}= & \frac{y_{j+1}-y_{j}}{h_{j}}-\frac{y_{j}-y_{j-1}}{h_{j-1}} \Rightarrow \\
h_{j-1} \frac{M_{j-1}}{2}+\left(h_{j-1}+h_{j}\right) M_{j}+h_{j} \frac{M_{j+1}}{2}= & 3\left(\frac{y_{j+1}-y_{j}}{h_{j}}-\frac{y_{j}-y_{j-1}}{h_{j-1}}\right) \Rightarrow \\
h_{j-1} c_{j-1}+2\left(h_{j-1}+h_{j}\right) c_{j}+h_{j} c_{j+1}= & 3\left(\frac{y_{j+1}-y_{j}}{h_{j}}-\frac{y_{j}-y_{j-1}}{h_{j-1}}\right), \\
& \text { for } j=1,2, \cdots, n-1
\end{aligned}
$$

Equation 1.37 can be simplified by setting:

$$
\begin{array}{r}
l_{j}=\frac{h_{j}}{h_{j-1}+h_{j}}, \quad k_{j}=1-l_{j}=\frac{h_{j-1}}{h_{j-1}+h_{j}}, \quad m_{j}=\frac{y_{j+1}-y_{j}}{h_{j}}, \\
n_{j}=\frac{3\left(m_{j}-m_{j-1}\right)}{h_{j-1}+h_{j}}, \quad c_{j}=\frac{M_{j}}{2}, \quad j=1,2, \ldots, n-1
\end{array}
$$

and

$$
k_{j} c_{j-1}+2 c_{j}+l_{j} c_{j+1}=n_{j}, \quad j=1,2, \ldots, n-1
$$

This equation can be used to form the system of $n-1$ linear equations. To find all parameters $c_{0}, c_{1}, \cdots, c_{n}$ it is required to have two more additional equations which can be written in the following form:

$$
\begin{aligned}
2 c_{0}+l_{0} c_{1} & =n_{0} \\
k_{n} c_{n-1}+2 c_{n} & =n_{n}
\end{aligned}
$$

The combined system of the linear equations in its general form becomes:

$$
\left[\begin{array}{ccccccc}
2 & l_{0} & 0 & \cdots & 0 & 0 & 0 \\
k_{1} & 2 & l_{1} & \cdots & 0 & 0 & 0 \\
0 & k_{2} & 2 & \cdots & 0 & 0 & 0 \\
\vdots & \vdots & \vdots & \vdots & \vdots & \vdots & \vdots \\
0 & 0 & 0 & \cdots & 2 & l_{n-2} & 0 \\
0 & 0 & 0 & \cdots & k_{n-1} & 2 & l_{n-1} \\
0 & 0 & 0 & \cdots & 0 & k_{n} & 2
\end{array}\right]\left[\begin{array}{c}
c_{0} \\
c_{1} \\
c_{2} \\
\vdots \\
c_{n-2} \\
c_{n-1} \\
c_{n}
\end{array}\right]=\left[\begin{array}{c}
n_{0} \\
n_{1} \\
n_{2} \\
\vdots \\
n_{n-2} \\
n_{n-1} \\
n_{n}
\end{array}\right]
$$

There are several possibilities for choosing values of the constants $l_{0}, n_{0}, k_{n}, n_{n}$ :

a) the simplest option is to choose $l_{0}=n_{0}=k_{n}=n_{n}=0$. This leads to $c_{0}=c_{n}=0$ and the equation for a natural cubic spline. The spline can be envisioned as a beam with simple supports at the ends; 
b) another option is to prescribe the slope of the spline at the ends, thus:

$$
S^{\prime}(a)=y_{0}^{\prime}, \quad S^{\prime}(b)=y_{n}^{\prime}
$$

These conditions are equivalent to selecting from equations 1.38:

$$
l_{0}=1=k_{n}, \quad n_{0}=\frac{6}{h_{1}}\left(\frac{y_{1}-y_{0}}{h_{1}}-y_{0}^{\prime}\right), \quad n_{n}=\frac{6}{h_{n}}\left(y_{n}^{\prime}-\frac{y_{n}-y_{n-1}}{h_{n}}\right)
$$

In this case it is required to define $y_{0}^{\prime}$ and $y_{n}^{\prime}$. They may be approximated using the derivatives of cubic interpolating polynomials based on the four points closest to the endpoints of the interval [9].

There exist some other ways of defining the constants $l_{0}, n_{0}, k_{n}, n_{n}$, for example, using the "not-to-knot" condition $[9,8]$. However, the first scheme is the simplest to apply and the resulting spline seems to give a good approximation of a function. Therefore, the determining equations for the natural cubic spline:

$$
\left[\begin{array}{ccccccc}
k_{1} & 2 & l_{1} & \cdots & 0 & 0 & 0 \\
0 & k_{2} & 2 & \cdots & 0 & 0 & 0 \\
\vdots & \vdots & \vdots & \vdots & \vdots & \vdots & \vdots \\
0 & 0 & 0 & \cdots & 2 & l_{n-2} & 0 \\
0 & 0 & 0 & \cdots & k_{n-1} & 2 & l_{n-1}
\end{array}\right]\left[\begin{array}{c}
c_{1} \\
c_{2} \\
\vdots \\
c_{n-2} \\
c_{n-1}
\end{array}\right]=\left[\begin{array}{c}
n_{1} \\
n_{2} \\
\vdots \\
n_{n-2} \\
n_{n-1}
\end{array}\right]
$$

Any solution procedure can be used to find $c_{1}, c_{2}, \ldots, c_{n-1}$ from the system of the linear equations 1.44. As soon as these parameters are found the equation for the cubic spline 1.34 is completely defined on every subinterval.

\subsubsection{Spline integration}

As soon as the spline that fits the points $\left(x_{i}, y_{i}\right)$ is found it is possible to integrate it formally to find the equation for spline integration. The derivation of the formula can be found in Appendix B. Integration on every subinterval $\left[x_{j-1}, x_{j}\right]$ can be performed using B-7:

$$
\int_{x_{j-1}}^{x_{j}} S(x) d x=\frac{y_{j-1}+y_{j}}{2} h_{j}-\frac{M_{j-1}+M_{j}}{24} h_{j}^{3}
$$

The final formula for the spline integration on $[a, b]$ becomes:

$$
\int_{a}^{b} S(x) d x=\sum_{j=1}^{n} \frac{y_{j-1}+y_{j}}{2} h_{j}-\sum_{j=1}^{n} \frac{M_{j-1}+M_{j}}{24} h_{j}^{3}
$$

Equation 1.45 can also be understood as the trapezoidal rule with a correction. 


\subsection{Gauss integration}

The trapezoidal and the Simpson's rules are using a low order polynomial approximation of the integrand $f(x)$ on subintervals that are decreasing in size. There is a class of integration methods that use polynomial approximations of $f(x)$ of increasing degree. These methods were developed for integrals in which the integrand has some kind of bad behaviour. In this case the integral is usually written as follows:

$$
I(f)=\int_{a}^{b} w(x) f(x) d x
$$

where $w(x)$ is the weight function which incorporates the bad behaviour and the function $f(x)$ is assumed to be well-behaved. The problem of finding $I(f)$ using numerical integration can now be written as follows:

$$
I(f)=\int_{a}^{b} w(x) f(x) d x \doteq \sum_{j=1}^{n} w_{j} f\left(x_{j}\right)=I_{n}(f)
$$

The weight function $w(x)$ is assumed to be nonnegative and integrable on $[a, b]$ and the major goal is to find the location of sampling points $x_{j}$ and the weights $w_{j}$ such that $I_{n}(f)$ equals $I(f)$ exactly for polynomials $f(x)$ of as high degree as possible. A simple case can be considered to explain the strategy of finding values of the nodes $x_{j}$ and the weights $w_{j}$. Let $w(x)=1$ and the integral becomes:

$$
I(f)=\int_{-1}^{1} f(x) d x \doteq \sum_{j=1}^{n} w_{j} f\left(x_{j}\right)
$$

The weights $w_{j}$ and the nodes $x_{j}$ have to be determined to make the integration error equal zero for a polynomial $f(x)$ of as high degree as possible:

$$
E_{n}(f)=\int_{-1}^{1} f(x) d x-\sum_{j=1}^{n} w_{j} f\left(x_{j}\right)=0
$$

If $f(x)$ is the polynomial of $m$ th degree the error formula can be written differently [8]:

$$
E_{n}\left(a_{0}+a_{1} x+\cdots+a_{m} x^{m}\right)=a_{0} E_{n}(1)+a_{1} E_{n}(x)+\cdots+a_{m} E_{n}\left(x^{m}\right)=0
$$

The error $E_{n}(f)$ is equal to zero for every polynomial of degree $\leq m$ if and only if

$$
E_{n}\left(x^{i}\right)=0, \quad \text { for } \quad i=0,1, \ldots, m
$$

For $n=1$ and only one node in the interval of integration there are two parameters $w_{1}$ and $x_{1}$ to be calculated from

$$
E_{n}(1)=0, \quad E_{n}(x)=0
$$


Which means that

$$
\int_{-1}^{1} 1 d x-w_{1}=0, \quad \int_{-1}^{1} x d x-w_{1} x_{1}=0
$$

Solving these equations one obtains $w_{1}=2$ and $x_{1}=0$. When the weights and nodes are determined one may say that the obtained integration rule is of Gauss type. As will be shown below, the type of the Gauss integration depends on the chosen weight function $w(x)$.

For a general $n$ there are $2 n$ parameters $x_{i}$ and $w_{i}$ to be defined and there exists an integration formula that uses $n$ nodes and gives a degree of precision of $2 n-1$. Similarly to the previous example, to find the required parameters the following equations will have to be solved [8]:

$$
E_{n}\left(x^{i}\right)=0, \quad i=0,1, \ldots, 2 n-1
$$

or

$$
\sum_{j=1}^{n} w_{j} x_{j}^{i}= \begin{cases}0, & i=1,3, \ldots, 2 n-1 \\ \frac{2}{i+1}, & i=0,2, \ldots, 2 n-2\end{cases}
$$

This is a set of nonlinear equations which can only be solved easily for a small number of the nodes. For a large or any number of the nodes another approach must be followed. This approach is based on the theory of orthogonal polynomials and is briefly described below. The complete theory of the orthogonal polynomials and some related computational procedures can be found in literature, see for example $[8,9,11]$.

Several terms are introduced first. Two functions are said to be orthogonal if their scalar product equals zero. A function is said to be normalised if its scalar product with itself equals unity. A set of functions that are mutually orthogonal and also all individually normalised is called an orthogonal set. Let $w(x) \geq 0$ be a fixed weight function defined on the interval $[a, b]$. It is possible to define a sequence of polynomials $\varphi_{0}(x), \varphi_{1}(x), \ldots$ which are orthogonal to $w(x)$ on $[a, b]$ :

$$
\int_{z}^{b} w(x) \varphi_{m}(x) \varphi_{n}(x) d x= \begin{cases}1, & \text { when } \mathrm{m}=\mathrm{n} \\ 0, & \text { when } \mathrm{m} \neq \mathrm{n}\end{cases}
$$

The polynomial $\varphi_{n}(x)$ can generally be represented as follows [11]:

$$
\varphi_{n}(x)=A_{n} \prod_{i=1}^{n}\left(x-x_{i}\right), \quad \text { with } \quad a<x_{1}<x_{2}<\ldots<x_{n}<b
$$

Where $A_{n}$ are some coefficients and $x_{i}$ are $n$ real roots of the polynomial. For $A_{n}>0$ and using some additional definitions:

$$
a_{n}=\frac{A_{n+1}}{A_{n}} \quad \text { and } \quad \gamma_{n}=\int_{a}^{b} w(x)\left[\varphi_{n}(x)\right]^{2} d x
$$


the following theorem can be presented [8].

Theorem 3: Let $f(x)$ be a function which is $2 n$ times continuously differentiable on $[a, b]$, a formula for $I_{n}(f)$ and its error can be given as follows:

$$
\begin{aligned}
I(f)= & \int_{a}^{b} w(x) f(x) d x \doteq \sum_{j=1}^{n} w_{j} f\left(x_{j}\right)+\frac{\gamma_{n}}{A_{n}^{2}(2 n) !} f^{2 n}(\eta)=I_{n}(f), \\
& \text { for some } a<\eta<b
\end{aligned}
$$

The nodes $x_{j}$ are zeros of $\varphi_{n}(x)$ and the weights $w_{j}$ are given by:

$$
w_{j}=\frac{-a_{n} \gamma_{n}}{\varphi_{n}^{\prime}\left(x_{j}\right) \varphi_{n+1}\left(x_{j}\right)}, \quad j=1, \ldots, n
$$

Proof of the theorem can be found in [8]. It is important to emphasise, that the roots of the orthogonal polynomials are the abscissas of the integration rule of Gauss type. In other words, when a set of polynomials orthogonal to a weight function is derived, their roots are found and the weights $w_{j}$ are calculated from equation 1.57 the Gauss rule for $n$ nodes is completely defined.

A set of orthogonal polynomials $\varphi_{n}(x)$ for a specific weight function $w(x)$ and an interval $[a, b]$ can be found using the triple recursion relation given by the following theorem [8].

Theorem 4. Let $\varphi_{n}$ be an orthogonal family of polynomials on $[a, b]$ with weight function $w(x) \geq 0$. Let it be defined as follows:

$$
\varphi_{n}(x)=A_{n} x^{n}+B_{n} x^{n-1}+\cdots, \quad \text { where } A_{n} \text { and } B_{n} \text { are some coefficients. }
$$

Then for $n \geq 1$

$$
\varphi_{n+1}(x)=\left(a_{n} x+b_{n}\right) \varphi_{n}(x)-c_{n} \varphi_{n-1}(x)
$$

with

$$
b_{n}=a_{n} \cdot\left[\frac{B_{n+1}}{A_{n+1}}-\frac{B_{n}}{A_{n}}\right] \quad c_{n}=\frac{A_{n+1} A_{n-1}}{A_{n}^{2}} \cdot \frac{\gamma_{n}}{\gamma_{n-1}}
$$

Proof of this theorem is given in [8]. The theorem allows finding a set of polynomials orthogonal to a weight function $w(x)$ on an interval $[a, b]$, provided that coefficients $b_{n}$ and $c_{n}$ are known. For most "classical" weight functions these coefficients are defined and formulae for generating orthogonal polynomials are readily available in the literature $[8,9,12]$. Table 1.1 presents a list of weight functions, intervals and recurrence relations that generate the most commonly used orthogonal polynomials. For an arbitrary weight function $w(x)$ on any interval $[a, b]$ coefficients $b_{n}$ and $c_{n}$ are not known and a set of orthogonal polynomials can be constructed using the Gram-Schmidt process [8,11].

Formulae for calculating the weights $w_{j}$ of the well-known orthogonal polynomials have also been defined. For example, in case of the Gauss-Legendre quadrature equation 1.57 becomes [13]:

$$
w_{j}=\frac{2}{\left(1-x_{j}^{2}\right)\left(P_{n}^{\prime}\left(x_{j}\right)\right)^{2}}
$$




\begin{tabular}{|c|c|c|c|}
\hline Name & Weight function & Interval & Recurrence relations \\
\hline \hline Legendre & 1 & {$[-1,1]$} & $(j+1) P_{j+1}=(2 j+1) x P_{j}-j P_{j-1}$ \\
Tschebyscheff & $\left(1-x^{2}\right)^{-\frac{1}{2}}$ & {$[-1,1]$} & $T_{j+1}=2 x T_{j}-T_{j-1}$ \\
Laguerre & $x^{\alpha} e^{-x}$ & {$[0, \infty]$} & $\begin{array}{c}(j+1) L_{j+1}^{\alpha}=(-x+2 j+\alpha+1) L_{j}^{\alpha}- \\
-(j+\alpha) L_{j-1}^{\alpha}\end{array}$ \\
& $e^{-x^{2}}$ & {$[-\infty, \infty]$} & $H_{j+1}=2 x H_{j}-2 j H_{j-1}$ \\
\hline
\end{tabular}

Table 1.1: Commonly used orthogonal polynomials.

where $P_{n}^{\prime}\left(x_{j}\right)$ is the derivative of the orthogonal polynomial at its zero $x_{j}$.

To summarise, the computation of the abscissas and the weights of the Gauss quadrature with an arbitrary number of the nodes comprises several phases:

- computation of the coefficients $b_{n}$ and $c_{n}$ and generation of a set of orthogonal polynomials. For well-known weight functions calculation of the coefficients can be omitted and the orthogonal polynomials can be derived from, for example, formulae in table 1.1;

- calculation of zeros $x_{j}$ of the orthogonal polynomials using a root-finding method;

- calculation of the associated weights $w_{j}$ using equation 1.59 .

To provide an example of using the theory presented above, the weights and the abscissas of the Gauss-Legendre quadrature with three integration points $(n=3)$ are calculated. As the first step, a set of the orthogonal polynomials is defined. Let $P_{-1}(x) \equiv 0$ and $P_{0}(x) \equiv 1$, then using the recurrence relation of the Legendre polynomials (see table 1.1) for $\mathrm{n}=3$ and $j=0,1,2$ :

$$
P_{1}=x, \quad P_{2}=\frac{3}{2} x^{2}-\frac{1}{2}, \quad P_{3}=\frac{5}{2} x^{3}-\frac{3}{2} x
$$

Roots of the $P_{3}(x)$ polynomial are

$$
x_{1}=0, \quad x_{2}=\sqrt{\frac{3}{5}}, \quad x_{3}=-\sqrt{\frac{3}{5}}
$$

These roots are the desired abscissas of the Gauss-Legendre quadrature. To calculate the weights it is necessary to find the derivative of the polynomial $P_{3}(x)$ :

$$
P_{3}^{\prime}(x)=\frac{15}{2} x^{2}-\frac{3}{2}
$$

Finally, from equation 1.59 one can calculate the weights:

$$
\begin{aligned}
w_{1} & =\frac{2}{\left(1-x_{1}^{2}\right)\left(P_{3}^{\prime}\left(x_{1}\right)\right)^{2}}=\frac{8}{9} \\
w_{2,3} & =\frac{2}{\left(1-x_{2}^{2}\right)\left(P_{3}^{\prime}\left(x_{2}\right)\right)^{2}}=\frac{10}{18}
\end{aligned}
$$


FORTRAN 90 subroutine gauleg which calculates the weights and the abscissas of the Gauss-Legendre quadrature with an arbitrary number of the integration points is given in Appendix C (source - Numerical Recipes in Fortran 90 [13]).

\subsection{Lobatto integration}

Lobatto integration can be considered as the integration formula of Gauss type with two preassigned nodes [9]:

$$
\int_{a}^{b} w(x) f(x) d x \approx \sum_{k=1}^{2} a_{k} f\left(y_{k}\right)+\sum_{k=1}^{n} w_{k} f\left(x_{k}\right)
$$

In this formula two nodes $y_{k}$ are fixed and prescribed in advance to lie on the limits of the integration interval $[a, b] .2+2 n$ constants $a_{k}, w_{k}$ and $x_{k}$ are to be determined so that the rule is exact for polynomials of the highest possible degree, which is $2+2 n-1=2 n+1$.

When $w(x)$ equals unity and $f(x)$ is $2 n-2$ times continuously differentiable on interval $[-1,1]$ the Lobatto rule becomes $[9]$ :

$$
\int_{-1}^{1} f(x) d x=\frac{2}{n(n-1)}[f(1)+f(-1)]+\int_{j=2}^{n-1} w_{j} f\left(x_{j}\right)
$$

Where $x_{j}$ are the roots of the Legendre polynomials $P(x)$. The weights $w_{j}$ can be determined as follows:

$$
w_{j}=\frac{2}{n(n-1)\left[P_{n-1}\left(x_{j}\right)\right]^{2}}, \quad \text { for } \quad x_{j} \neq \pm 1
$$

The algorithm for calculating the abscissas $x_{j}$ and the weights $w_{j}$ of the Lobatto rule can be found in [14]. The tabulated abscissas and weights of the Lobatto rule with up to 20 integration points can also be found in [15].

The major advantage of the Lobatto rule over the Gauss quadrature is the presence of sampling points on the limits of the integration interval. This fact can be very important for through-thickness integration in shell elements, since the Lobatto rule can take into account an initiation of yielding at the sheet surface.

\subsection{Choice of an integration rule for shell elements}

All presented numerical procedures can be used to integrate through the thickness of plates and shells and they are usually available in commercial finite element packages. Their use depends on a problem and each of them has its advantages and disadvantages.

The rules based on the Newton-Cotes formula are simple to implement since the entire step of calculation of weights and nodal locations can be omitted. However, if the function to be integrated is nonlinear and continuous on $[a, b]$ then the Gauss quadrature and the 
Lobatto rule are more preferable. The speed of convergence of these rules increases with increasing integrand smoothness. In contrast, the trapezoidal and the Simpson's rules will not converge faster than the specific level.

Presence of integration points on the interval's limits may give extra advantages. When an element is bent to a certain radius, yielding of a material will be initiated in the outer surfaces and the Lobatto rule can directly identify that. For the same situation, the outermost Gauss integration points may still be in the elastic regime and, as a result, the internal bending moment will be overestimated. The Lobatto rule, however, requires more integration points to achieve the accuracy similar to the Gauss integration. For example, when a material is in the elastic regime, the Gauss quadrature will give the exact solution for the bending moment with only two points and the Lobatto rule will need three points.

If a function to be integrated has discontinuities on $[a, b]$ the advantages of the considered integration rules diminish. Burgoyne and Crisfield [3] investigated performance of various numerical strategies in integrating stresses through the thickness of plates and shells when there are discontinuities in stress profiles. Using four different problems they showed that all the rules do not perform well when using less than 10 integration points. The Gauss quadrature was recommended as the most accurate one. Additionally, it was noted that in some cases it may be preferable to use a simpler integration strategy, such as the trapezoidal rule. Depending on the stress profile, due to error cancellation, accuracy of a simple rule can be comparable to that of the Gauss quadrature with similar number of integration points.

In the analysis of Burgoyne and Crisfield simple stress profiles through only half of the plate thickness were considered leading to the necessity of using even more integration points in general cases. An alternative numerical scheme is needed that is more suitable for integrating functions with discontinuities and which can guarantee accurate results with a low number of points independently of the stress profile complexity. To develop such a scheme one has to investigate performance of the traditional numerical schemes in more general situations when the integrand discontinuities are changing in their shape and number. 



\section{Chapter 2}

\section{Origins of numerical integration error}

\subsection{Analytical model of bending of a beam under ten- sion}

To understand performance of numerical strategies in integrating a function with discontinuities a simple problem of bending of a beam under in-plane tension is considered. The analytical model that represents this problem is developed below. The model allows calculation of the internal bending moment in a beam bent to a certain radius under in-plane tension. The analytical model is based on following assumptions:

- normal section planes remain plane and normal to the middle surface (Kirchhoff hypothesis);

- the plane strain condition exists in the bending plane;

- the plane stress situation is assumed;

- bends with a constant curvature are considered. Within the bends radial $\rho$ and circumferential directions $\theta$ can be distinguished. Due to the Kirchhoff hypothesis the radial and the circumferential directions are the principal strain directions.

\subsubsection{Strains and stresses}

Bending of a sheet material over a tool radius $R$ with a superimposed tension is considered (figure 2.1). As can be seen in this figure, due to the applied tension $T$ the neutral line shifts towards the curvature centre. Variable $a$ describes the position of the neutral line. The total circumferential strain consists of two parts: the tensile strain $\varepsilon_{m}$ and the bending strain $\varepsilon_{b}$. The tensile strain has a constant value in a cross-section and is equal to:

$$
\varepsilon_{m}=-\frac{a}{\rho}
$$



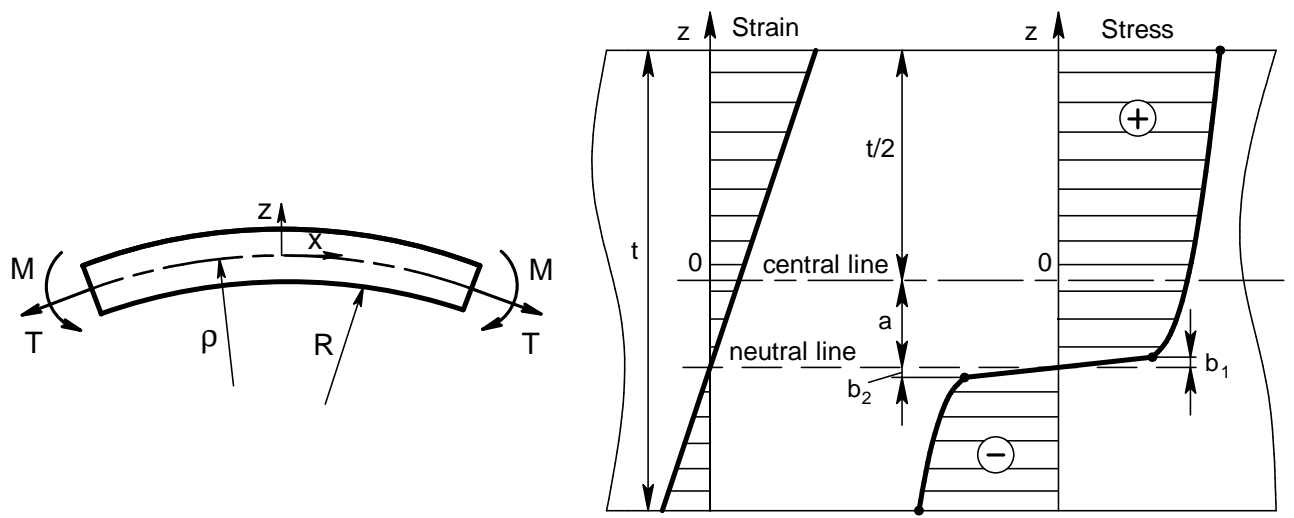

Figure 2.1: Stretch bending. Distribution of strains and stresses through the thickness.

where $\rho=R+t / 2$ and $t$ is the material thickness.

Assuming that the bending strain is $\varepsilon_{b}=z / \rho$, the total circumferential strain becomes:

$$
\varepsilon_{\theta}=\frac{z-a}{\rho}
$$

Variables $b_{1}$ and $b_{2}$ shown in figure 2.1 are used to describe a position of yield points in tension and compression regions. The coordinates of the yield points can be found as follows:

$$
\begin{aligned}
& z_{1}=a+b_{1} \\
& z_{2}=a-b_{2}
\end{aligned}
$$

Substituting equations 2.2 into equation 2.1 the yield strains in tension and compression regions can be found:

tension region

$$
\varepsilon_{\theta}^{y}=\frac{z_{1}-a}{\rho} \Rightarrow \varepsilon_{\theta}^{y}=\frac{a+b_{1}-a}{\rho} \Rightarrow \varepsilon_{\theta}^{y}=\frac{b_{1}}{\rho}
$$

compression region

$$
\varepsilon_{\theta}^{y}=\frac{z_{2}-a}{\rho} \Rightarrow \varepsilon_{\theta}^{y}=\frac{a-b_{2}-a}{\rho} \Rightarrow \varepsilon_{\theta}^{y}=-\frac{b_{2}}{\rho}
$$

In the elastic region the circumferential stress is found from the Hook's law for plane strain:

$$
\sigma_{\theta}^{e}=\frac{E}{1-\nu^{2}} \varepsilon_{\theta}^{e}=\frac{E}{1-\nu^{2}} \frac{z-a}{\rho}
$$

where $E$ is the Young's modulus and $\nu$ is the Poisson's ratio. Using the expression for the Hill'48 yield function in plane strain, the value of the circumferential strain at yield can be found as follows:

$$
\varepsilon_{\theta}^{y}=\frac{\sigma_{y 0}\left(1-\nu^{2}\right)}{E}=\frac{\frac{2}{\sqrt{3}} \sigma_{u n}\left(1-\nu^{2}\right)}{E}
$$


where $\sigma_{y 0}$ is the initial yield stress and $\sigma_{u n}$ is the uniaxial yield stress. Making the simplifying substitution, $C_{1}=\frac{2}{\sqrt{3}}\left(1-\nu^{2}\right)$, the circumferential yield strain becomes:

$$
\varepsilon_{\theta}^{y}=\frac{C_{1} \sigma_{u n}}{E}
$$

Equation 2.6 can be used to find the position of the yield points $b_{1}$ and $b_{2}$ for a particular material and a curvature of bending. Combining equations 2.3 and 2.4 with the yield strain equation 2.6 one obtains:

$$
\begin{gathered}
\frac{b_{1}}{\rho}=\frac{C_{1} \sigma_{u n}}{E} \Rightarrow b_{1}=\frac{C_{1} \sigma_{u n} \rho}{E} \\
-\frac{b_{2}}{\rho}=-\frac{C_{1} \sigma_{u n}}{E} \Rightarrow b_{2}=\frac{C_{1} \sigma_{u n} \rho}{E}
\end{gathered}
$$

The total circumferential strain in the region of plastic deformations can be defined as the sum of two parts - the constant strain at yield and the plastic strain due to material workhardening:

$$
\varepsilon_{\theta}=\varepsilon_{\theta}^{y}+\varepsilon_{\theta}^{p}
$$

Rewriting the foregoing equation, the plastic strain due to the material workhardening can be derived:

tension region

$$
\begin{aligned}
\varepsilon_{\theta}=\varepsilon_{\theta}^{y}+\varepsilon_{\theta}^{p} & \Rightarrow \frac{z-a}{\rho}=\frac{C_{1} \sigma_{u n}}{E}+\varepsilon_{\theta}^{p} \Rightarrow \\
\varepsilon_{\theta}^{p} & =\frac{z-a}{\rho}-\frac{C_{1} \sigma_{u n}}{E}
\end{aligned}
$$

compression region

$$
\begin{gathered}
\varepsilon_{\theta}=\varepsilon_{\theta}^{y}+\varepsilon_{\theta}^{p} \Rightarrow \frac{z-a}{\rho}=-\frac{C_{1} \sigma_{u n}}{E}+\varepsilon_{\theta}^{p} \Rightarrow \\
\varepsilon_{\theta}^{p}=\frac{z-a}{\rho}+\frac{C_{1} \sigma_{u n}}{E}
\end{gathered}
$$

The circumferential stress in the region of plastic deformations can be approximated by a power law:

$$
\sigma_{\theta}=C^{\prime}\left(\varepsilon_{\theta}^{p}\right)^{n}
$$

where $C^{\prime}=C\left(\frac{2}{\sqrt{3}}\right)^{(n+1)}$ and $C$ and $n$ are parameters that describe the material workhardening. Substituting the plastic strain equations 2.9 and 2.10 into equation 2.11 it is possible to obtain formulae that describe the plastic part of the circumferential stress in tension and compression regions: 
tension region

$$
\sigma_{\theta}^{p}=C^{\prime}\left(\varepsilon_{0}+\frac{z-a}{\rho}-\frac{C_{1} \sigma_{u n}}{E}\right)^{n}
$$

compression region

$$
\sigma_{\theta}^{p}=-C^{\prime}\left|\varepsilon_{0}+\frac{z-a}{\rho}+\frac{C_{1} \sigma_{u n}}{E}\right|^{n}
$$

where $\varepsilon_{0}$ is a pre-strain which can be found from the following condition:

$$
\sigma_{y 0}=C^{\prime} \varepsilon_{0}^{n}
$$

\subsubsection{Stress resultants}

Using the circumferential stresses it is possible to find forces and bending moments acting on the sheet per unit length:

$$
\begin{gathered}
T=\int_{-t / 2}^{t / 2} \sigma_{\theta} d z \\
M=\int_{-t / 2}^{t / 2} \sigma_{\theta} z d z
\end{gathered}
$$

To simplify the derivation the tension applied at the middle plane is split into four components:

$$
T=T^{e}+C^{e}+T^{p}+C^{p}
$$

In this equation $T^{e}$ and $C^{e}$ are the tensile and the compressive forces caused by the elastic stresses. $T^{p}$ and $C^{p}$ are the tensile and the compressive forces caused by the plastic stresses. The contribution of the elastic and the plastic stresses to the total tension will be:

tension region

$$
\begin{gathered}
T^{e}=\int_{a}^{z_{1}} \frac{E}{1-\nu^{2}} \frac{z-a}{\rho} d z \\
T^{p}=\int_{z_{1}}^{\frac{t}{2}} C^{\prime}\left(\varepsilon_{0}+\frac{z-a}{\rho}-\frac{C_{1} \sigma_{u n}}{E}\right)^{n} d z
\end{gathered}
$$

compression region

$$
\begin{gathered}
C^{e}=\int_{z_{2}}^{a} \frac{E}{1-\nu^{2}} \frac{z-a}{\rho} d z \\
C^{p}=-\int_{-\frac{t}{2}}^{z_{2}} C^{\prime}\left|\varepsilon_{0}+\frac{z-a}{\rho}+\frac{C_{1} \sigma_{u n}}{E}\right|^{n} d z
\end{gathered}
$$

The full derivation of these equations can be found in Appendix C. Final formulae that can be used for calculating the total tension and the resulting shift of the neutral line are presented below.

$$
T^{e}=\frac{C_{1}^{2} \sigma_{u n}^{2} \rho}{2\left(1-\nu^{2}\right) E}
$$




$$
\begin{gathered}
T^{p}=\frac{C^{\prime} \rho}{n+1}\left(\left(\varepsilon_{0}+\frac{\frac{t}{2}-a}{\rho}-\frac{C_{1} \sigma_{u n}}{E}\right)^{n+1}-\varepsilon_{0}^{n+1}\right) \\
C_{e}=-\frac{C_{1}^{2} \sigma_{u n}^{2} \rho}{2\left(1-\nu^{2}\right) E} \\
C^{p}=-\frac{C^{\prime} \rho}{n+1}\left|\varepsilon_{0}^{n+1}-\right| \frac{-\frac{t}{2}-a}{\rho}+\frac{C_{1} \sigma_{u n}}{E}-\left.\varepsilon_{0}\right|^{n+1} \mid
\end{gathered}
$$

The total moment per unit width acting about the middle plane can be described as follows:

$$
M=M_{T}^{e}+M_{T}^{p}+M_{C}^{e}+M_{C}^{p}
$$

where $M_{T}^{e}$ and $M_{T}^{p}$ are the elastic and the plastic parts of the total bending moment in the region of tension. $M_{C}^{e}$ and $M_{C}^{p}$ are the elastic and the plastic parts of the total bending moment in the region of compression. The parts of the total bending moment can be found as follows:

tension region

$$
\begin{gathered}
M_{T}^{e}=\int_{a}^{z_{1}} \frac{E}{1-\nu^{2}}\left(\frac{z-a}{\rho}\right) z d z \\
M_{T}^{p}=\int_{z_{1}}^{\frac{t}{2}} C^{\prime}\left(\varepsilon_{0}+\frac{z-a}{\rho}-\frac{C_{1} \sigma_{u n}}{E}\right)^{n} z d z
\end{gathered}
$$

compression region

$$
\begin{gathered}
M_{C}^{e}=\int_{z_{2}}^{a} \frac{E}{1-\nu^{2}}\left(\frac{z-a}{\rho}\right) z d z \\
M_{C}^{p}=-\int_{-\frac{t}{2}}^{z_{2}} C^{\prime}\left|\varepsilon_{0}+\frac{z-a}{\rho}+\frac{C_{1} \sigma_{u n}}{E}\right|^{n} z d z
\end{gathered}
$$

The closed form solution of the foregoing equations is presented in Appendix C. Final expressions for the parts of the total bending moment are presented below:

$$
\begin{gathered}
M_{T}^{e}=\frac{C_{1}^{2} \sigma_{u n}^{2} \rho}{E\left(1-\nu^{2}\right)}\left(\frac{C_{1} \sigma_{u n} \rho}{3 E}+\frac{a}{2}\right) \\
M_{T}^{p}=C^{\prime} \rho\left(\frac{\rho}{n+2}\left(\left(\frac{\frac{t}{2}-a}{\rho}-\frac{C_{1} \sigma_{u n}}{E}+\varepsilon_{0}\right)^{n+2}-\varepsilon_{0}^{n+2}\right)+\left(\frac{C_{1} \sigma_{u n} \rho}{E}-\varepsilon_{0} \rho+a\right) \frac{1}{n+1}\right. \\
\left.\left(\left(\frac{\frac{t}{2}-a}{\rho}-\frac{C_{1} \sigma_{u n}}{E}+\varepsilon_{0}\right)^{n+1}-\varepsilon_{0}^{n+1}\right)\right)
\end{gathered}
$$




$$
\begin{gathered}
M_{C}^{e}=\frac{C_{1}^{2} \sigma_{u n}^{2} \rho}{E\left(1-\nu^{2}\right)}\left(\frac{C_{1} \sigma_{u n} \rho}{3 E}-\frac{a}{2}\right) \\
M_{C}^{p}=C^{\prime} \rho \mid \frac{\rho}{n+2}\left(\varepsilon_{0}^{n+2}-\left|\frac{-\frac{t}{2}-a}{\rho}+\frac{C_{1} \sigma_{u n}}{E}-\varepsilon_{0}\right|^{n+2}\right)-\left(\varepsilon_{0} \rho+a-\frac{C_{1} \sigma_{u n} \rho}{E)}\right) \frac{1}{n+1} \times \\
\\
\left(\varepsilon_{0}^{n+1}-\left|\frac{-\frac{t}{2}-a}{\rho}+\frac{C_{1} \sigma_{u n}}{E}-\varepsilon_{0}\right|^{n+1}\right) \mid
\end{gathered}
$$

\subsubsection{Change of curvature - springback}

During unloading, when external loads are removed the deformed sheet springs back to a different shape. The expression for a curvature change can be found by considering the change of internal stresses due to the elastic unloading:

$$
\begin{aligned}
\Delta \sigma_{\theta} & =\frac{E}{1-\nu^{2}} \Delta \varepsilon_{\theta}, \quad \text { where } \\
\Delta \varepsilon_{\theta} & =\frac{z}{\rho}-\frac{z}{\rho^{\prime}}=\Delta\left(\frac{1}{\rho}\right) z
\end{aligned}
$$

$\rho^{\prime}$ is a radius after unloading. The change in internal stresses causes the change in bending moment, $\triangle M$ :

$$
\begin{gathered}
\Delta M=2 \int_{0}^{t / 2} \Delta \sigma_{\theta} z d z=2 \int_{0}^{t / 2} \frac{E}{1-\nu^{2}} \Delta\left(\frac{1}{\rho}\right) z^{2} d z \quad \Rightarrow \\
\Delta M=\frac{E t^{3}}{12\left(1-\nu^{2}\right)} \Delta\left(\frac{1}{\rho}\right)=\frac{t^{3}}{12} \frac{\Delta \sigma_{\theta}}{z}
\end{gathered}
$$

If the bending moment is $M=M_{T}^{e}+M_{T}^{p}+M_{C}^{e}+M_{C}^{p}$ the removal of the external loads results in $\Delta M=-M$. Therefore the change of shape during the unloading can be obtained:

$$
\begin{aligned}
& \frac{E t^{3}}{12\left(1-\nu^{2}\right)} \Delta\left(\frac{1}{\rho}\right)=-M \\
& \Delta\left(\frac{1}{\rho}\right)=-\frac{12 M\left(1-\nu^{2}\right)}{E t^{3}}
\end{aligned}
$$

The last equation shows that the change of curvature $(\Delta 1 / \rho)$ or springback is proportional to the bending moment $\mathrm{M}$. Therefore, an error in calculating the moment can be considered as the measure of accuracy of a springback calculation. 


\subsubsection{Influence of in-plane tension on springback}

To show what effect an increase in tension has on the amount of springback a simplified model of bending of a beam under tension is considered. In this model the elastic, perfectly plastic material is used. The assumptions introduced in section 2.1 are also applied here. At first the beam is bent elastically. The resulting bending moment can be found from 2.15:

$$
M=\int_{-\frac{t}{2}}^{\frac{t}{2}} \frac{E}{1-\nu^{2}} \frac{z}{\rho} z d z=\frac{E t^{3}}{12\left(1-\nu^{2}\right) \rho}=\frac{\sigma_{\theta} t^{3}}{12 z}
$$

The elastic limit is reached when $\sigma_{\theta}$ reaches the plane strain yield stress $\sigma_{y 0}=\left(2 / \sqrt{3} \sigma_{u n}\right)$ at the outer fibers. The corresponding radius of bending is called limiting bending radius $\rho_{e}$. The elastic limiting moment can be defined as follows:

$$
M_{e}=\frac{E t^{3}}{12\left(1-\nu^{2}\right) \rho_{e}}=\frac{\sigma_{y 0} t^{2}}{6}
$$

The ratio between the current moment $M$ and the elastic limiting moment $M_{e}$ :

$$
\frac{M}{M_{e}}=\frac{\rho_{e}}{\rho}
$$

When the tension is applied after applying the bending loads the circumferential stress $\sigma_{\theta}$ will be a superimposition of bending and tensile stresses:

$$
\sigma_{\theta}=\sigma_{b}+\sigma_{t}=\frac{12 M z}{t^{3}}+\sigma_{t}
$$

The tensile stress needed to cause plastic deformations in the outer fiber of the beam (at $z=t / 2)$ is defined as follows:

$$
\sigma_{t}=\sigma_{\theta}-\frac{12 M z}{t^{3}}=\sigma_{y 0}-\frac{6 M}{t^{2}}
$$

Therefore, the tension needed to cause the plastic deformation:

$$
T=\sigma_{y 0} t-\frac{6 M}{t}=T_{y}\left(1-\frac{6 M}{\sigma_{y 0} t^{2}}\right)=T_{y}\left(1-\frac{\rho_{e}}{\rho}\right)
$$

As soon as the tension exceeds this value the bending moment will start to reduce. At some moment, the yield point is at distance $m t / 2$ from the central line, see figure 2.2 , where $-1<m<1$. At this point the circumferential strain is equal to the yield strain:

$$
\varepsilon_{\theta}=\varepsilon_{m}+\varepsilon_{b}=\varepsilon_{m}+\frac{z}{\rho}=\frac{\sigma_{y 0}\left(1-\nu^{2}\right)}{E}
$$




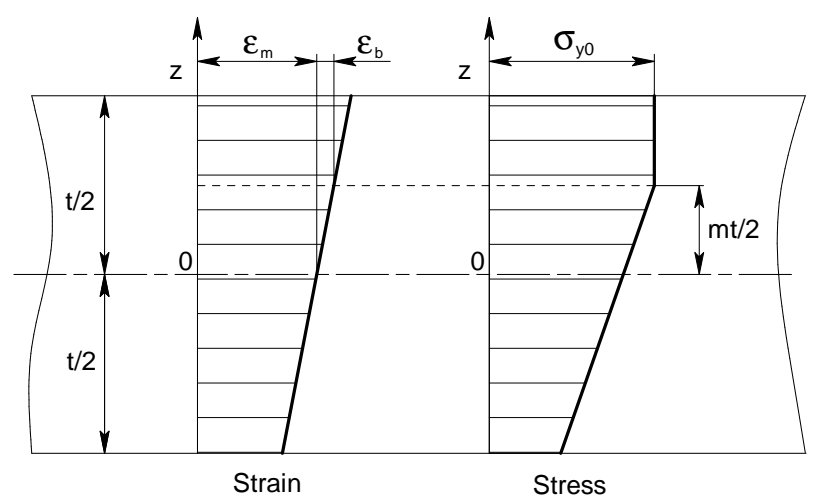

Figure 2.2: Distribution of strains and stresses in elastic, perfectly plastic beam bent to a radius and stretched.

The tensile part of the total strain becomes:

$$
\varepsilon_{m}=\frac{\sigma_{y 0}\left(1-\nu^{2}\right)}{E}-\frac{m t}{2 \rho}
$$

The circumferential strain at a distance $z$ from the central line can be written as follows:

$$
\varepsilon_{\theta}=\frac{\sigma_{y 0}\left(1-\nu^{2}\right)}{E}-\frac{m t}{2 \rho}+\frac{z}{\rho}
$$

The stress distribution in the elastic region can be found from the Hook's law:

$$
\sigma_{\theta}^{e}=\frac{E}{1-\nu^{2}} \varepsilon_{\theta}=\sigma_{y 0}+\frac{E}{\left(1-\nu^{2}\right) \rho}\left(z-\frac{m t}{2}\right)
$$

Using equation 2.15 one can find the resulting bending moment:

$$
\begin{aligned}
M & =\int_{-\frac{t}{2}}^{\frac{m t}{2}}\left(\sigma_{y 0}+\frac{E}{\left(1-\nu^{2}\right) \rho}\left(z-\frac{m t}{2}\right)\right) z d z+\int_{\frac{m t}{2}}^{\frac{t}{2}} \sigma_{y 0} z d z= \\
& =\left.\sigma_{y 0} \frac{z^{2}}{2}\right|_{-\frac{t}{2}} ^{\frac{m t}{2}}+\frac{E}{\left(1-\nu^{2}\right) \rho}\left(\left.\frac{z^{3}}{3}\right|_{-\frac{t}{2}} ^{\frac{m t}{2}}-\left.\frac{m t z^{2}}{4}\right|_{-\frac{t}{2}} ^{\frac{m t}{2}}\right)+\left.\sigma_{y 0} \frac{z^{2}}{2}\right|_{\frac{m t}{2}} ^{\frac{t}{2}}= \\
& =\frac{E}{\left(1-\nu^{2}\right) \rho}\left(\frac{m^{3} t^{3}}{24}+\frac{t^{3}}{24}-\frac{m^{3} t^{3}}{16}+\frac{m t^{3}}{16}\right)=\frac{E t^{3}}{\left(1-\nu^{2}\right) \rho}\left(\frac{2+3 m-m^{3}}{4}\right)= \\
& =M_{e}\left(\frac{2+3 m-m^{3}}{4}\right)
\end{aligned}
$$

Increasing the tension will cause the yield point move inwards and, as can be seen from equation 2.47, the bending moment will decrease. Thus, if due to tools geometry or process conditions the beam is fully plastic, no elastic springback will take place.

\subsubsection{Results of analytical calculations}

A number of calculations is performed to test the developed analytical model. The calculations are done with various values of the in-plane tension for two different materials, 
namely IF steel and aluminium alloy AL5182. Thickness of the beam is $1 \mathrm{~mm}$ and material parameters are summarised in table 2.1 .

\begin{tabular}{|c|c|c|c|c|c|}
\hline & $\mathrm{E}, \mathrm{GPa}$ & $\nu$ & $\sigma_{y 0}, \mathrm{MPa}$ & $\mathrm{C}, \mathrm{MPa}$ & $\mathrm{n}$ \\
\hline IF steel & 210 & 0.3 & 150 & 425 & 0.4 \\
\hline AL5182 & 70.6 & 0.341 & 125.02 & 561.34 & 0.321 \\
\hline
\end{tabular}

Table 2.1: Material properties for IF steel and AL5182.

Calculated values of the bending moment plotted versus the neutral line shift are shown in figure 2.3. As expected, the bending moment decreases with increasing amount of the in-plane tension.

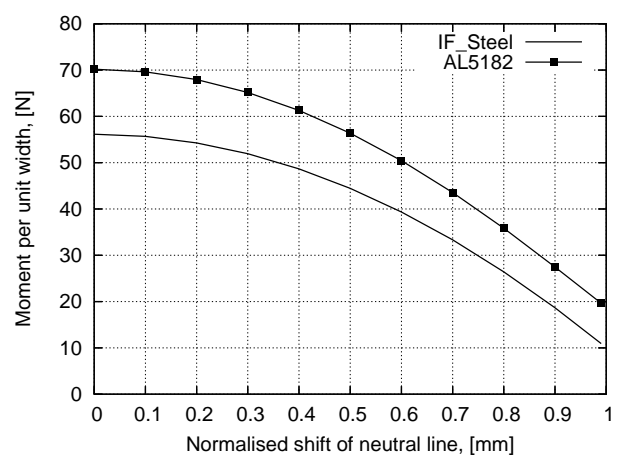

Figure 2.3: Dependency of bending moment on neutral line shift.

Through-thickness distribution of the circumferential stresses which occur in the beam bent to the radii of $100 \mathrm{~mm}$ and $5 \mathrm{~mm}$ is shown in figures $2.4(\mathrm{a})$ and $2.4(\mathrm{~b})$. The material

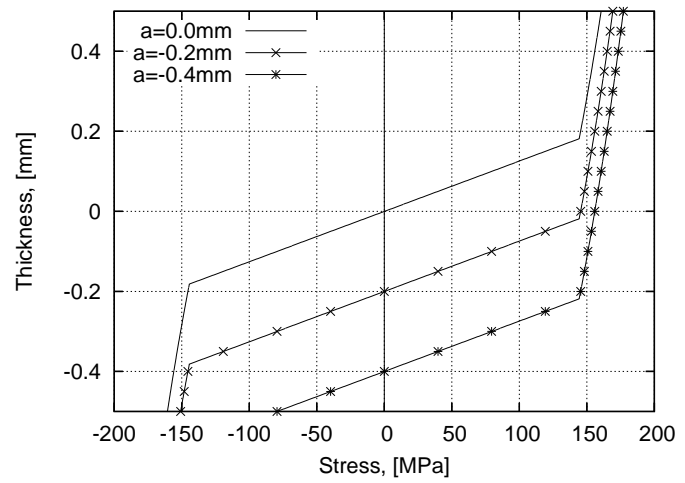

(a) $\mathrm{R} / \mathrm{t}=100$

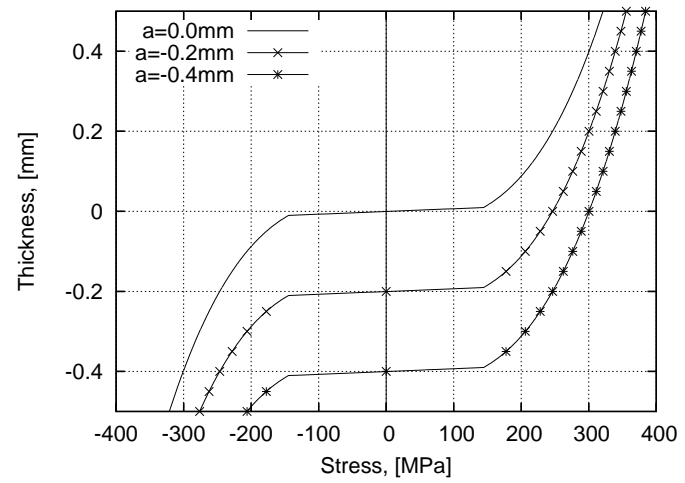

(b) $\mathrm{R} / \mathrm{t}=5$

Figure 2.4: Stress profiles for various process conditions.

of the beam is AL5182 and the calculations are performed with various values of in-plane tension. The applied tension causes the neutral line shift equal to $0.0 \mathrm{~mm},-0.2 \mathrm{~mm}$ and $-0.4 \mathrm{~mm}$. From these figures it can be seen that bending the beam to a smaller radius results into much higher values of the stresses. 
The integrand $\sigma_{\theta} \cdot z$ from the integral for calculating the bending moment (see equation $2.15)$ is plotted in figures 2.5(a) and 2.5(b). The integrand's profile intersects the $\mathrm{x}$ axis at the points which coincide with the locations of the neutral line, since the stresses at these points are equal to zero. It can be seen that increasing the shift of the neutral line and decreasing the radius of bending makes the integrand's profile less smooth and its points of discontinuity become more pronounced.

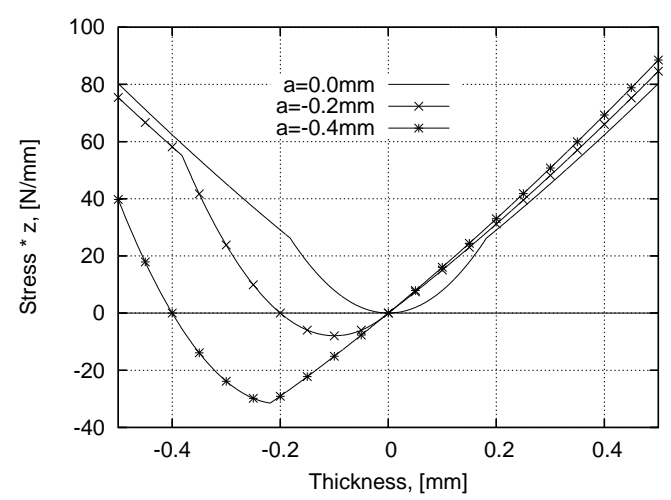

(a) $\mathrm{R} / \mathrm{t}=100$

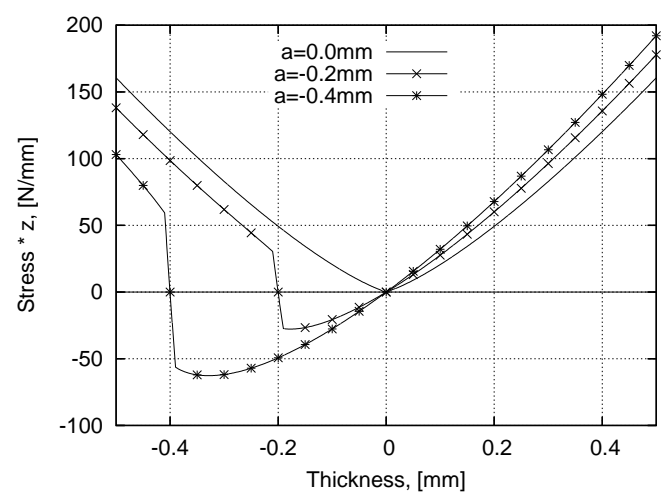

(b) $\mathrm{R} / \mathrm{t}=5$

Figure 2.5: Integrand profiles for various process conditions.

\subsection{Numerical calculation of bending moment}

Influence of the numerical integration error on the accuracy of calculation of springback is evaluated in this section. Two integration schemes, namely the trapezoidal rule and the Gauss quadrature, are used to calculate the integral 2.15:

$$
M_{\text {num }}=\frac{1}{2} \sum_{j=1}^{n} w_{j} \sigma_{\theta}\left(\xi_{j}\right) \xi_{j}
$$

where $n$ is the number of the integration points, $\sigma_{\theta}\left(\xi_{j}\right)$ is the integration point's value of the circumferential stress and $\xi_{j}$ are the through-thickness locations of the integration points. The error due to applying the numerical integration is quantified by finding a relative difference between values of the bending moment calculated analytically and numerically.

$$
\mathrm{RME}=\frac{M_{\text {num }}-M_{\text {analytical }}}{M_{\text {analytical }}} \cdot 100 \%
$$

where RME is the relative moment error. The first set of calculations is performed for the beam of $1 \mathrm{~mm}$ thickness. The material of the beam is IF steel, the bending radius is $5 \mathrm{~mm}$ and the neutral line shift is $-0.4 \mathrm{~mm}$. Dependency of the relative moment error on the number of the integration points for the trapezoidal rule and the Gauss quadrature is shown in figure 2.6. The Gauss quadrature performs better than the trapezoidal rule and 
converges faster to a saturated value of the moment error. At the same time, due to the complexity and the non-smoothness of the integrand, both rules lead to a considerably high error when using $3-10$ integration points. It can also be seen that the relative moment error oscillates with changing the number of the integration points. The oscillation of the moment error, which is best visible in figure 2.6(a), can be explained by a more preferable location of the integration points relative to the position of the points of discontinuity in the integrand's profile [16]. In other words, changing the integration points number changes their through-thickness position and at some instant an integration point lies in the vicinity of the point of discontinuity leading to a drastic decrease in the integration error.

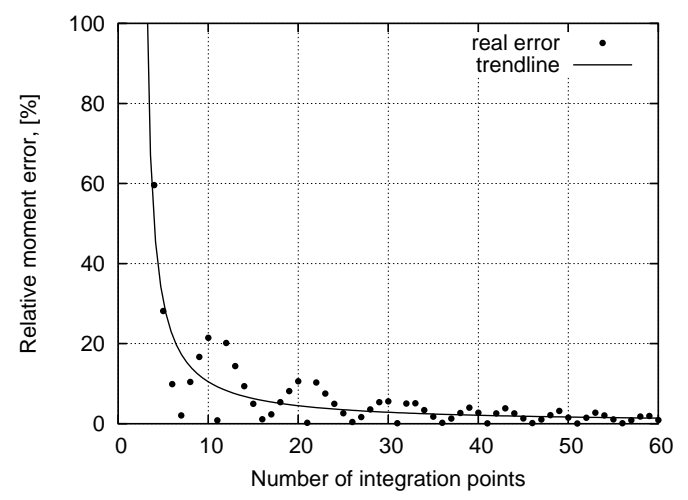

(a) Trapezoidal rule

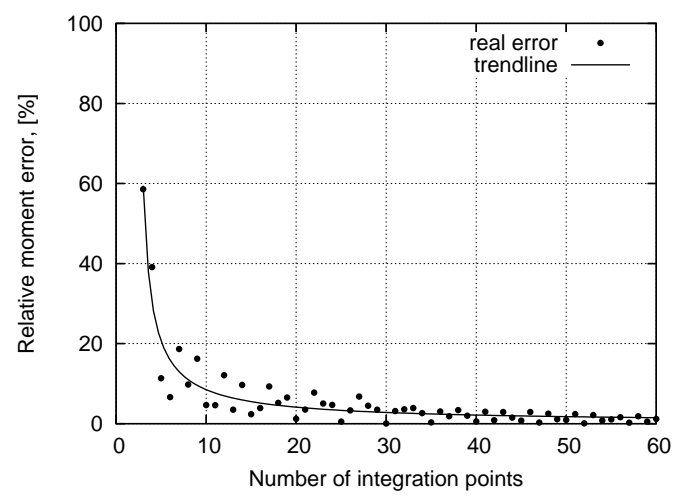

(b) Gauss rule

Figure 2.6: Relative moment error due to numerical integration.

As was shown earlier, the integrand profile changes with variation of process parameters, such as, for example, the in-plane tension or the $\mathrm{R} / \mathrm{t}$ ratio. Variation of these parameters leads to a change of the integrand smoothness, especially near the points of discontinuity. Apparently, the integration error, that depends on the integrand profile, will also be a function of the process parameters. To ascertain key factors that have an influence on the level of the integration error, several sets of calculations are performed in which the $\mathrm{R} / \mathrm{t}$ ratio and amount of the in-plane tension are varied. The material of the beam is IF steel and the trapezoidal rule with 50 integration points is used in these calculations.

The relative moment error as a function of the in-plane tension for $R / t=5$ is shown in figure 2.7. The tension in this figure and in all subsequent figures is represented by the normalised shift of the neutral line, which is defined as the neutral line shift divided by a half of the beam thickness:

$$
\bar{a}=\frac{2 a}{t}
$$

As can be seen the error due to numerical integration oscillates and increases with increasing tension. Once again, the oscillation can be explained by more favourable or less favourable position of the integration points relative to the points of discontinuity in the integrand profile. In this case the location of the integration points is fixed, and the points of discontinuity are moving through the thickness with the increasing tension. The increase 


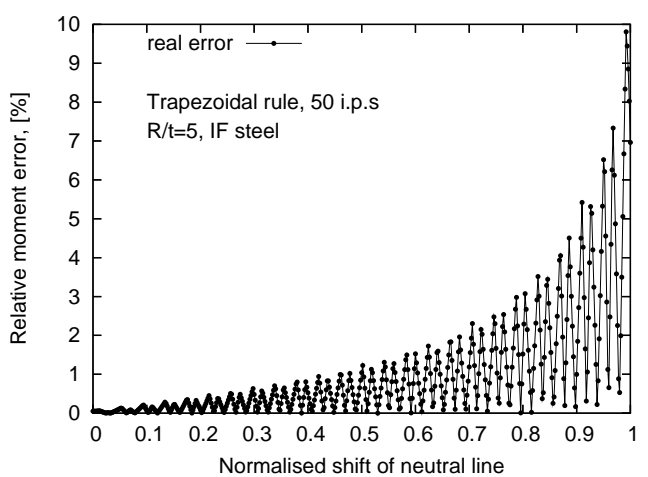

Figure 2.7: Variation of relative moment error with increasing in-plane tension.

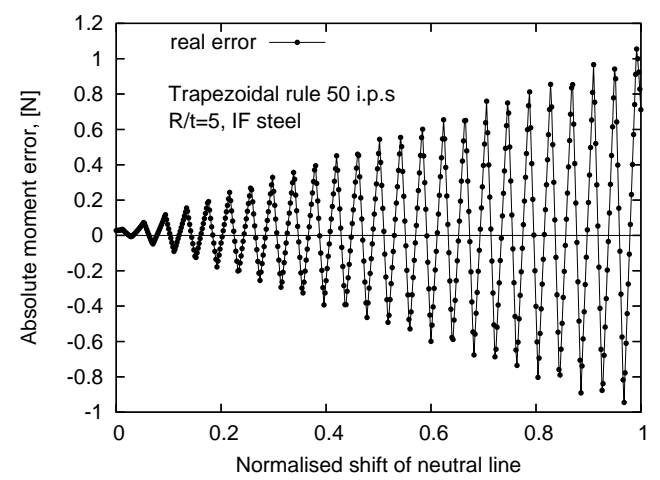

Figure 2.8: Variation of absolute moment error with increasing in-plane tension.

of the numerical integration error can be explained by recalling the fact that the bending moment decreases with increasing tension. Therefore, a given absolute moment error due to numerical integration will lead to a larger relative error. At the same time, the absolute moment error is not constant, as can be seen in figure 2.8. It increases with the increasing tension since the integral profile becomes less smooth.

Influence of the $\mathrm{R} / \mathrm{t}$ ratio on the relative moment error under constant tension is shown in figure 2.9. The calculations are performed using the trapezoidal rule. Due to the oscillation a term maximum error is introduced. It is an extreme value of the relative moment error for a given set of the process conditions. Figure 2.9 shows that when the bending radius is sharp the relative moment error is larger and more integration points are needed to assure a certain accuracy level.

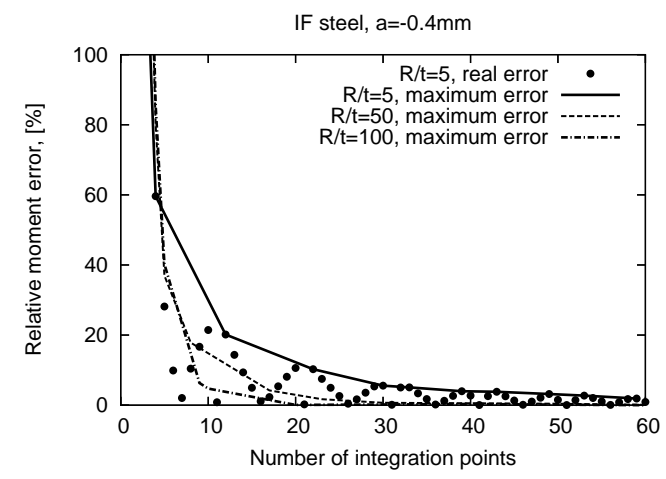

Figure 2.9: Relative moment error as a function of $\mathrm{R} / \mathrm{t}$ ratio.

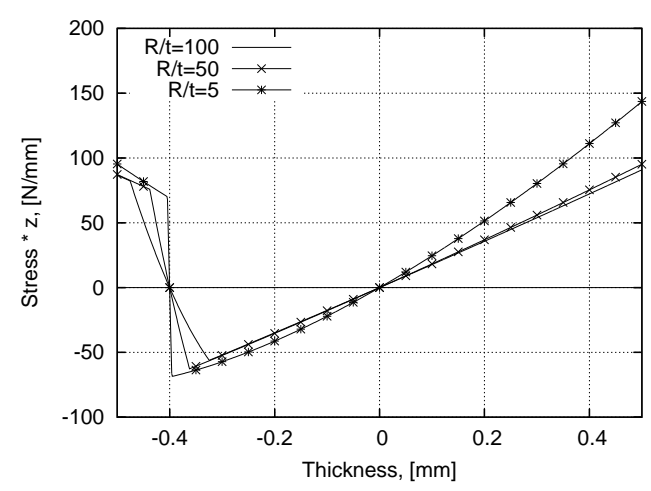

Figure 2.10: Integrand profiles for various $\mathrm{R} / \mathrm{t}$ ratios. IF Steel.

The observed increase in the relative moment error when using smaller bending radii can be explained with the help of figure 2.10, where the through-thickness integrand profiles are plotted for various $\mathrm{R} / \mathrm{t}$ ratios. Two trends are visible in this plot. Decreasing the bending radius makes the integrand profile more curved in tension and compression regions and more sharp near the points of discontinuity. To understand if these trends are responsible for the larger relative moment error similar calculations are performed for Al5182 (see 
figures 2.11 and 2.12). It can be seen that despite the higher curvature in the integrand's profile the relative moment error is smaller for AL5182 (compare the curves with $R / t=5$ in figures 2.11 and 2.9). The integrand profile near the points of discontinuity, however, is less sharp and can be a reason of the relative moment error being smaller for the aluminium alloy comparing to IF steel. As a result, one may conclude that mainly the shape of the integral profile near the points of discontinuity is responsible for variation of the relative moment error with changing process parameters.

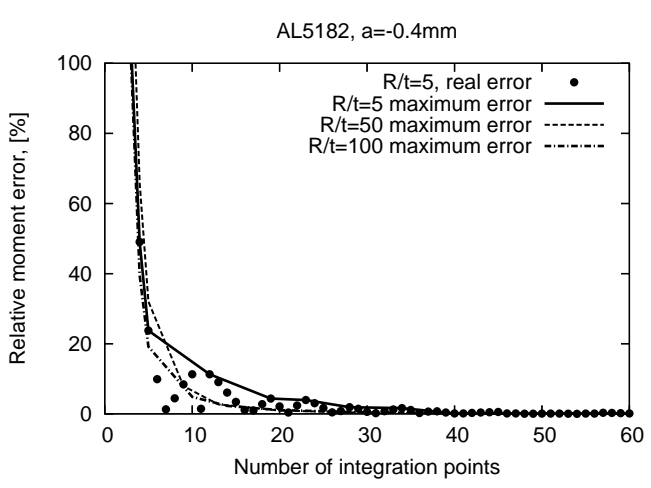

Figure 2.11: Relative moment error as a function of $\mathrm{R} / \mathrm{t}$ ratio.

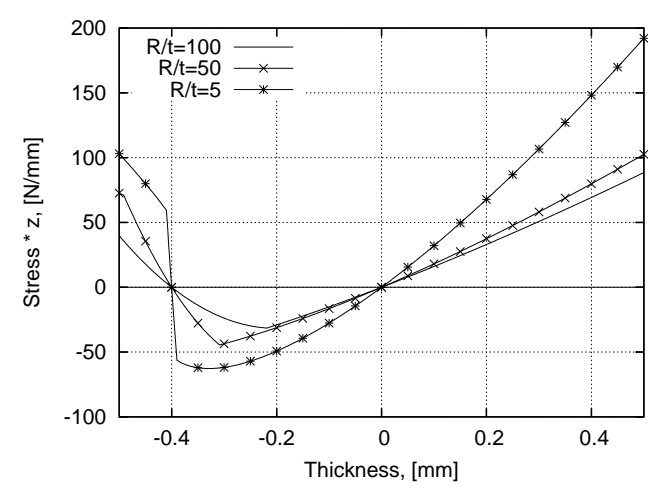

Figure 2.12: Integrand profiles for various $\mathrm{R} / \mathrm{t}$ ratios. AL5182.

To complete this section and to show what influence the error due to the numerical integration may have on the shape after springback one may consider a real example of bending of a strip under tension. The material of the strip is IF steel and its thickness is $1 \mathrm{~mm}$. The radius of bending is $20 \mathrm{~mm}$ and the strip length is $100 \mathrm{~mm}$. The middle part of the strip is subjected to bending. Forming angle, which is the angle between the free edge of the strip and the central line, is $45^{\circ}$. The change of curvature during springback, calculated from equation 2.36 using the analytical value of the bending moment, is shown in figure 2.13 for varying value of tension. Additionally, the change of curvature, found using the numerically calculated bending moment, is plotted in this figure. The numerical integration is performed with the Gauss quadrature using 7 integration points. The global trend reduction of the curvature change by increasing the tension - can be easily explained. The change of curvature is proportional to the bending moment which decreases with increasing the tension (as can be seen in figure 2.3). In addition to this trend, the curve obtained using the numerical bending moment is oscillatory. There are three plateaus where the curvature change is almost constant. To explain this fact the numerical moment is plotted as a function of tension, see figure 2.15. Five different values of tension are considered, marked in this figure with $a, b, c, d$ and $e$. These values are used to calculate the integrand's profiles that are shown in figure 2.16. The through-thickness location of all seven integration points is highlighted with the dashed lines. Increasing the tension makes the points of discontinuity in the integrand's profile move with respect to the integration points. Before they reach the closest integration point (point 3 in figure 2.16) the bending moment value decreases only slightly, since in the vicinity of every sampling point the integrand's profile undergoes only small modifications with changing the process parameters. As soon as the integration point 3 falls into the elastic region (profile b) its contribution to the bending 


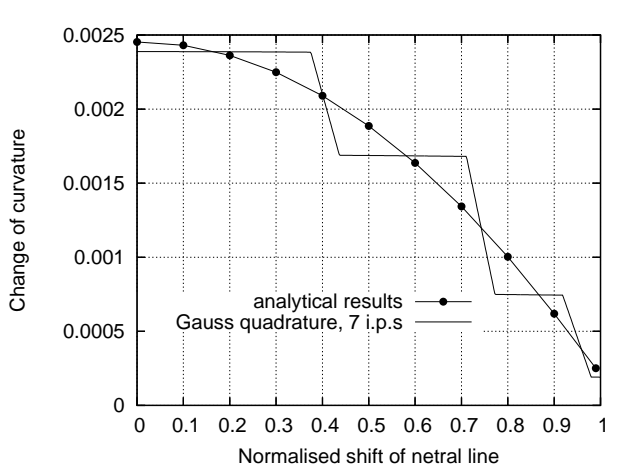

Figure 2.13: Change of curvature as a function of in-plane tension. $R / t=20, \mathrm{IF}$ steel.

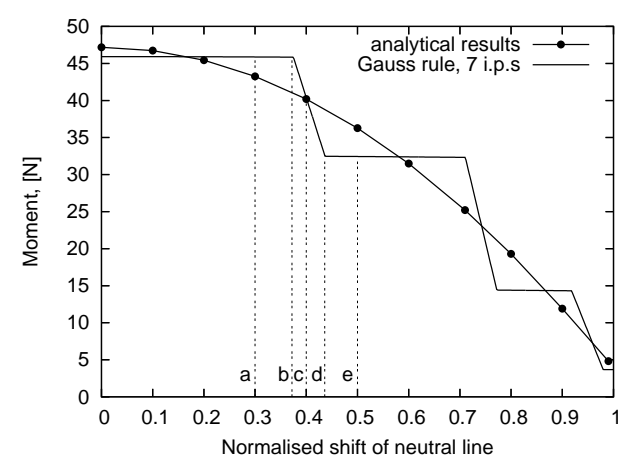

Figure 2.15: Numerical bending moment as a function of in-plane tension. $R / t=$ 20, IF steel.

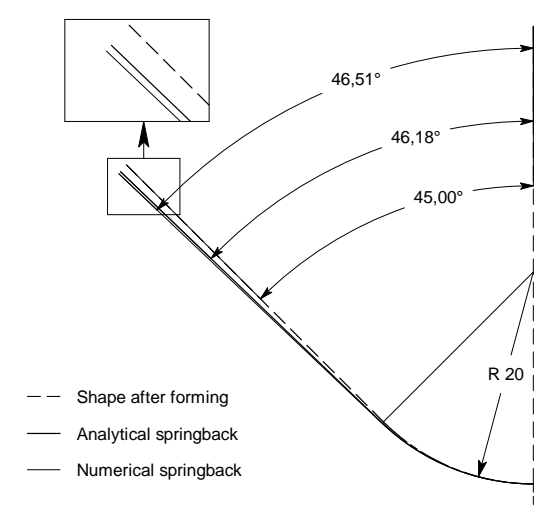

Figure 2.14: Shape of the strip after forming and springback.

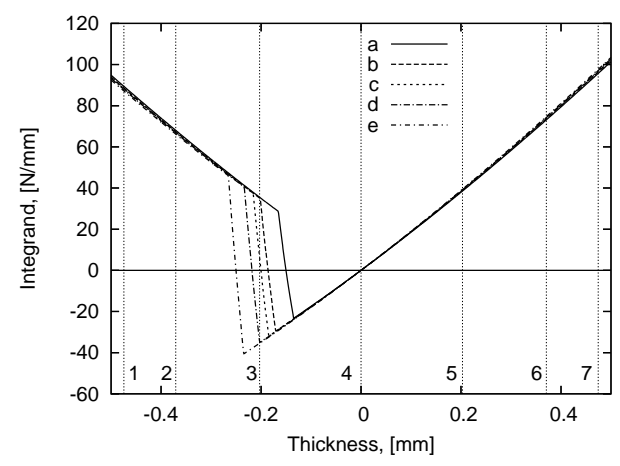

Figure 2.16: Integrand's profiles for different values of tension.

moment value decreases abruptly and after becomes negative. As a result the value of the bending moment drops sharply (see figure 2.15). Soon after the integration point 3 passes the elastic region (profile $\mathrm{d}$ ) the moment value stabilises and remains relatively constant until the integration point 2 falls into the elastic region.

The oscillation of the bending moment can result in under- or over-estimation of the change of shape during springback. For example, let 0.71 be a value of the normalised shift of the neutral line (see figure 2.13). The analytical and the numerical values of the curvature change are $1.3109 \mathrm{e}-03$ and $1.6808 \mathrm{e}-03$ correspondingly. These values together with the initial curvature and the forming angle can be used to build the shape of the strip after forming and springback which is shown in figure 2.14. In this figure, the analytical springback corresponds to the shape of the strip which is built using the analytical value of the curvature change. The numerical springback is the shape based on the numerical value of the curvature change. The error due to numerical integration is responsible for the overestimation of the angle after springback and this increase is about $28 \%$ of the total, analytically calculated change of the angle. Underestimation of the springback angle will take place if the normalised shift of the neutral line is 0.75 . 


\subsection{Error due to numerical integration}

By using the simple problem of bending of a beam under tension it is shown that the commonly used integration rules require up to 20 integration points to assure a low value of the error due to numerical integration. For deformation regimes which occur in sheet metal forming the through-thickness stress profile may be more complex leading to even higher number of the integration points.

If an integration rule uses integration points that are fixed in thickness direction the error due to numerical integration is oscillatory in nature. It is related to more favourable or less favourable position of the integration points relative to the points of discontinuity in the stress profile. This fact makes it impossible to develop practical guidelines for choosing an appropriate number of the integration points. For a particular problem, depending on process conditions and material parameters a fixed number of the integration points may lead to a very high or a very low integration error.

Presence of the points of discontinuity in the stress profile diminishes advantages of various integration rules with the fixed location of sampling points. Both the trapezoidal rule and the Gauss quadrature require a large number of the integration points to obtain negligible numerical integration error.

The error due to numerical integration depends on the smoothness of the integrand profile near the points of discontinuity. A higher concentration of the integration points in this region will lead to a smaller error. A curvature at other regions of the integrand profile has second order effects on the integration error and its influence can be cancelled by using higher order rules.

It is clear that, due to the presence of the discontinuities in the integrand profile, traditional integration rules are not so effective and an alternative numerical scheme is needed. Such an alternative can be an adaptive strategy that uses algorithms to choose abscissas and weights depending on integrand's properties and, thus, can perform accurate integration while using a small number of the integration points. 



\section{Chapter 3}

\section{Adaptive integration scheme for bending with tension problem}

In this chapter an adaptive integration strategy for the simple problem of bending of a beam under tension is developed. The main characteristic feature of the strategy is the ability to find the accurate numerical solution of an integral while using a limited number of integration points. In the beginning, the existing numerical schemes for adaptive integration are described and their advantages and disadvantages are discussed. The most suitable strategy is chosen and then further developed to be applicable to the bending with tension problem. After, the adaptive strategy is tested and compared with the traditional integration schemes.

\subsection{Rules for adaptive integration}

In addition to the global integration rules, described in chapter 1, there are numerical schemes that use an adaptive strategy. The integration rule in these schemes varies placement of its points to reflect a changing local behaviour of the integrand. Adaptive quadrature schemes are as effective and efficient for well-behaved integrands as the traditional integration rules. Furthermore, they are equally effective and efficient for a broad range of badly-behaved integrands where the traditional formulae are ineffective. All adaptive integration schemes can be classified as iterative and noniterative [9].

In an iterative scheme, successive approximations to an integral are computed until the final result is satisfactory within a given tolerance. Plenty of adaptive iterative algorithms have been developed, see for example $[17,18,19,20]$, with the main goal to compute an integral value as accurately as possible with no strong restriction on the amount of integration points used for that purpose. A global description of this group of adaptive schemes is given below:

1) to find an integral value an initial number of integration points is chosen and a global integration rule is applied to each panel. The panel is a part of the integration interval 
which consists of a certain number of the integration points, for example three points for the Simpson's rule;

2) the error estimate is found on every panel and if it is higher than a specified value this panel is subdivided into a number of sub-panels. Several subdivision strategies are available starting with a simple halving till increasing the number of points with optimising their location. The detailed description of some strategies for the interval subdivision can be found in $[20,21,22]$;

3) the same global integration rule is applied on every sub-panel. Alternatively, a higher order rule can be applied on the entire panel or a group of sub-panels;

4) each new sub-panel is subdivided as many times as necessary until the error estimate is within a given tolerance;

5) the final value of the integral is a found by adding the integral values on every subpanel.

In a noniterative scheme, prior to calculating the final integral value the integrand is evaluated to choose the most optimal location and the number of the integration points. This scheme, in contrast to an iterative one, is focused on finding the accurate integral value using a limited number of the integration points. Therefore, it is the best candidate to be used for numerical calculation of integrals in the bending with tension problem.

The framework for constructing an adaptive integration scheme was suggested by Rice [17]. It was formulated that the meta-algorithm for an adaptive integration consists of several groups of components, namely: interval processor components, algorithm controller components and interval collection management components. The meta-algorithm is presented graphically in figure 3.1. In this case an interval is more than just two points. It also in-

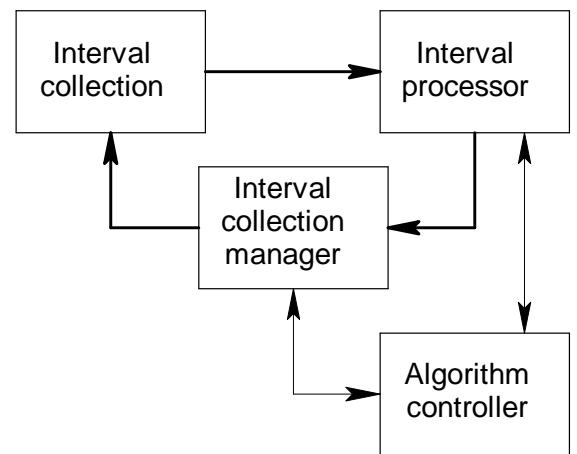

Figure 3.1: Meta-algorithm for adaptive integration. Solid lines show flow of intervals and light lines show flow of control and other information [17].

cludes all additional information associated with this interval, for example coordinates of the endpoints and their integrand values, error information and some auxiliary data. The interval collection is organised into some data structure which is created and maintained by the interval collection manager. Intervals are taken from the collection and the interval 
processor performs all required computations associated with each interval, for example integration and error estimation. The interval processor may use different integration rules or their combinations. The overall error estimate and its distribution is calculated by components of the algorithm controller. Additionally, components of the algorithm controller calculate presence and location of singularities and discontinuities in the integrand profile. Finally, components of the interval collection manager define and organise new intervals in a way suitable for subsequent calculations. An adaptive algorithm can be constructed by choosing from each block specific components that depend on characteristics of a problem.

\subsection{Definition of adaptive noniterative strategy}

The meta-algorithm of Rice can be used to develop an adaptive integration scheme for calculating the bending moment in a beam which is bent under tension (see figure 2.1). It is modified to yield the algorithm which has a unidirectional flow of information. This modification is possible since one seeks to develop the noniterative adaptive scheme. Some components of the algorithm controller and the interval collection manager are grouped into a new block which is called interval manager. The second block, the interval processor, is left unchanged. The main blocks of the adaptive noniterative scheme are shown in figure 3.2 and their components are described in greater detail below.

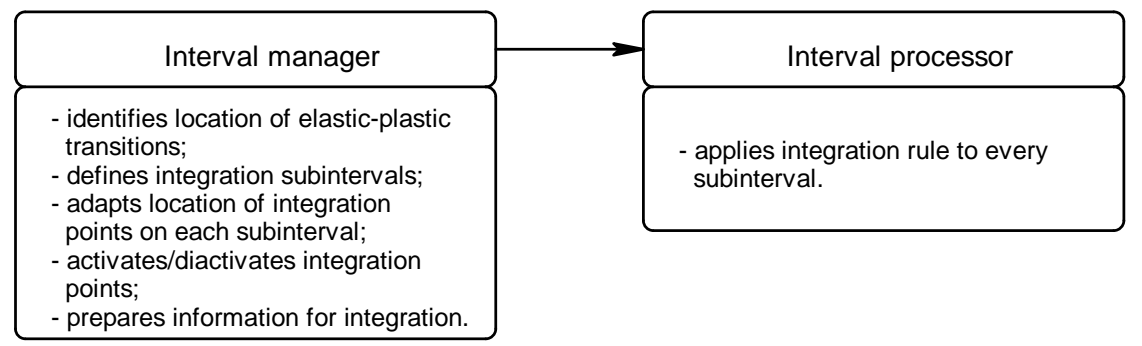

Figure 3.2: Block diagram of adaptive noniterative algorithm.

\subsubsection{Interval manager}

The main task of the interval manager is to evaluate an integrand prior to numerical integration. It includes several components that are required to:

- identify location of points of discontinuity in the integrand profile. For the bending with tension problem the discontinuities are the points where the material transits from elastic into plastic. The locations of these transition points can be calculated analytically using equations 2.7 and 2.8 ;

- subdivide the integration interval into subintervals. Figure 3.3 shows the integrand profile for IF steel and a particular set of process conditions: $R / t=5, t=1 \mathrm{~mm}$, 
neutral line shift $=-0.2 \mathrm{~mm}$. Points $\mathrm{B}$ and $\mathrm{C}$ mark the location of the discontinuities and $\mathrm{AB}, \mathrm{BC}$ and $\mathrm{CD}$ are the subintervals;

- activate or deactivate the integration points and adapt their location. The integration points inside of every subinterval are marked with the crosses and on the subinterval's limits they are marked with the solid dots. Their location and number depends on integration rule and is adapted to improve the accuracy of integration;

- prepare information for subsequent integration. Depending on the integration rule that will be used on every subinterval weights and some other parameters must be recalculated.

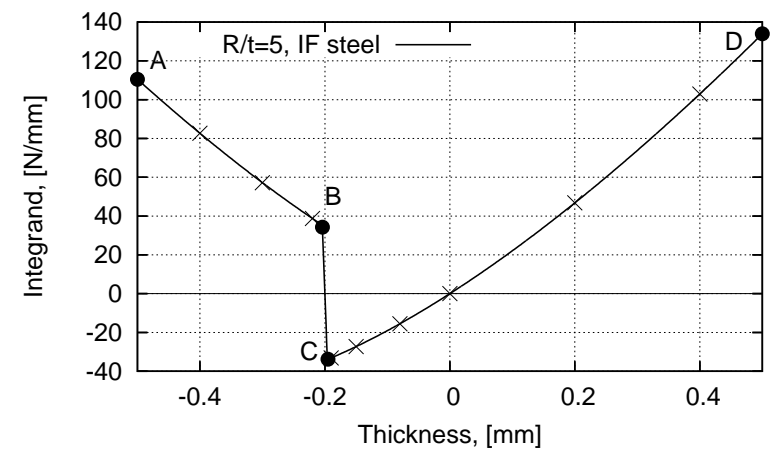

Figure 3.3: Subdivision of integration interval.

\subsubsection{Interval processor}

The integration points on every subinterval do not necessarily have to be equally spaced. It is known that for low order rules, such as for example the trapezoidal rule, curvature of the integrand profile influences the integration error. The error increases with the increasing curvature. Therefore, it is highly possible that when using a simple rule the interval manager will create a higher concentration of the integration points near the points of discontinuity, where the curvature is the highest. Thus, a numerical scheme is needed that is capable of performing integration using unequally distributed integration points.

The idea behind integration on unequally distributed points is similar to that of the Newton-Cotes integration. As the first step, an integrand $f(x)$ is approximated by an interpolating function $p(x)$ on integration points $a \leq x_{1}, x_{2}, \ldots, x_{n} \leq b$. After that, solving analytically the integral $\int_{a}^{b} p(x) d x$ gives the formula for integration with the unequally distributed points. Various functions can be used for approximation, for example a polynomial or a natural cubic spline. Some integration formulae are developed below. 


\section{Generalised composite trapezoidal rule}

Let $a \leq x_{0}, x_{1}, \ldots, x_{n} \leq b$ be a set of unequally spaced abscissas with $y_{0}, y_{1}, \ldots, y_{n}$ their corresponding functional values. The linear polynomial that approximates $f(x)$ on two consecutive points $x_{j-1}, x_{j}$ for $j=1,2, \ldots, n$ can be written as follows:

$$
\begin{aligned}
p_{1}(x) & =\frac{x-x_{j}}{x_{j-1}-x_{j}} y_{j-1}+\frac{x-x_{j-1}}{x_{j}-x_{j-1}} y_{j}=\frac{y_{j-1} x-y_{j-1} x_{j}+y_{j} x_{j-1}-y_{j} x}{x_{j-1}-x_{j}}= \\
& =\frac{y_{j-1}-y_{j}}{x_{j-1}-x_{j}} x+\frac{y_{j} x_{j-1}-y_{j-1} x_{j}}{x_{j-1}-x_{j}}
\end{aligned}
$$

The formula for numerical integration with generalised composite trapezoidal rule then becomes:

$$
\begin{aligned}
I_{n}(f) & =\sum_{j=1}^{n} \int_{x_{j-1}}^{x_{j}} p_{1}(x) d x=\sum_{j=1}^{n} \int_{x_{j-1}}^{x_{j}}\left(\frac{y_{j-1}-y_{j}}{x_{j-1}-x_{j}} x+\frac{y_{j} x_{j-1}-y_{j-1} x_{j}}{x_{j-1}-x_{j}}\right) d x= \\
& =\sum_{j=1}^{n}\left(\left.\frac{y_{j-1}-y_{j}}{2\left(x_{j-1}-x_{j}\right)} x^{2}\right|_{x_{j-1}} ^{x_{j}}+\left.\frac{y_{j} x_{j-1}-y_{j-1} x_{j}}{x_{j-1}-x_{j}} x\right|_{x_{j-1}} ^{x_{j}}\right)= \\
& =\sum_{j=1}^{n}\left(\frac{y_{j-1}-y_{j}}{2\left(x_{j-1}-x_{j}\right)}\left(x_{j}^{2}-x_{j-1}^{2}\right)+\frac{y_{j} x_{j-1}-y_{j-1} x_{j}}{x_{j-1}-x_{j}}\left(x_{j}-x_{j-1}\right)\right)= \\
& =\sum_{j=1}^{n}\left(\frac{\left(y_{j}-y_{j-1}\right)\left(x_{j}+x_{j-1}\right)}{2}-y_{j} x_{j-1}+y_{j-1} x_{j}\right)= \\
& =\sum_{j=1}^{n}\left(\frac{y_{j} x_{j}-y_{j-1} x_{j}+y_{j} x_{j-1}-y_{j-1} x_{j-1}-2 y_{j} x_{j-1}+2 y_{j-1} x_{j}}{2}\right)= \\
& =\sum_{j=1}^{n}\left(\frac{y_{j} x_{j}+y_{j-1} x_{j}-y_{j} x_{j-1}-y_{j-1} x_{j-1}}{2}\right) \Rightarrow \\
I_{n}(f) & =\sum_{j=1}^{n}\left(\frac{1}{2}\left(x_{j}-x_{j-1}\right)\left(y_{j}+y_{j-1}\right)\right)
\end{aligned}
$$

If the abscissas are equally spaced and $h=x_{j}-x_{j-1}$, then equation 3.2 transforms into the formula for the composite trapezoidal rule (see equation 1.16).

\section{Generalised composite Simpson's rule}

Let $a \leq x_{0}, x_{1}, \ldots, x_{n} \leq b$ be a set of unequally spaced abscissas with $y_{0}, y_{1}, \ldots, y_{n}$ their corresponding functional values. Quadratic polynomial that approximates $f(x)$ on three consecutive points $x_{j-1}, x_{j}, x_{j+1}$ for $j=1,2, \ldots, n-1$ can be written as follows:

$$
\begin{aligned}
p_{2}(x) & =\frac{\left(x-x_{j}\right)\left(x-x_{j+1}\right)}{\left(x_{j-1}-x_{j}\right)\left(x_{j-1}-x_{j+1}\right)} y_{j-1}+\frac{\left(x-x_{j-1}\right)\left(x-x_{j+1}\right)}{\left(x_{j}-x_{j-1}\right)\left(x_{j}-x_{j+1}\right)} y_{j}+ \\
& +\frac{\left(x-x_{j-1}\right)\left(x-x_{j}\right)}{\left(x_{j+1}-x_{j-1}\right)\left(x_{j+1}-x_{j}\right)} y_{j+1}
\end{aligned}
$$


For simplicity of derivation a particular case is considered. The quadratic polynomial on $x_{0}, x_{1}, x_{2}$ becomes:

$$
\begin{aligned}
p_{2}(x) & =\frac{\left(x-x_{1}\right)\left(x-x_{2}\right)}{\left(x_{0}-x_{1}\right)\left(x_{0}-x_{2}\right)} y_{0}+\frac{\left(x-x_{0}\right)\left(x-x_{2}\right)}{\left(x_{1}-x_{0}\right)\left(x_{1}-x_{2}\right)} y_{1}+\frac{\left(x-x_{0}\right)\left(x-x_{1}\right)}{\left(x_{2}-x_{0}\right)\left(x_{2}-x_{1}\right)} y_{2}= \\
& =\frac{\left(x-x_{1}\right)\left(x-x_{2}\right)}{\left(x_{0}-x_{1}\right)\left(x_{0}-x_{2}\right)} y_{0}-\frac{\left(x-x_{0}\right)\left(x-x_{2}\right)}{\left(x_{0}-x_{1}\right)\left(x_{1}-x_{2}\right)} y_{1}+\frac{\left(x-x_{0}\right)\left(x-x_{1}\right)}{\left(x_{0}-x_{2}\right)\left(x_{1}-x_{2}\right)} y_{2}= \\
& =\frac{\left(x-x_{1}\right)\left(x-x_{2}\right)\left(x_{1}-x_{2}\right) y_{0}-\left(x-x_{0}\right)\left(x-x_{2}\right)\left(x_{0}-x_{2}\right) y_{1}}{\left(x_{0}-x_{1}\right)\left(x_{0}-x_{2}\right)\left(x_{1}-x_{2}\right)}+ \\
& +\frac{\left(x-x_{0}\right)\left(x-x_{1}\right)\left(x_{0}-x_{1}\right) y_{2}}{\left(x_{0}-x_{1}\right)\left(x_{0}-x_{2}\right)\left(x_{1}-x_{2}\right)}=\frac{\left(x^{2}-x\left(x_{1}+x_{2}\right)+x_{1} x_{2}\right)\left(x_{1}-x_{2}\right) y_{0}}{\left(x_{0}-x_{1}\right)\left(x_{0}-x_{2}\right)\left(x_{1}-x_{2}\right)}- \\
& -\frac{\left(x^{2}-x\left(x_{0}+x_{2}\right)+x_{0} x_{2}\right)\left(x_{0}-x_{2}\right) y_{1}}{\left(x_{0}-x_{1}\right)\left(x_{0}-x_{2}\right)\left(x_{1}-x_{2}\right)}+\frac{\left.\left(x_{0}+x_{1}\right)+x_{0} x_{1}\right)\left(x_{0}-x_{1}\right) y_{2}}{\left(x_{0}-x_{1}\right)\left(x_{0}-x_{2}\right)\left(x_{1}-x_{2}\right)}= \\
& =\frac{\left(x_{1}-x_{2}\right) y_{0}-\left(x_{0}-x_{2}\right) y_{1}+\left(x_{0}-x_{1}\right) y_{2}}{\left(x_{0}-x_{1}\right)\left(x_{0}-x_{2}\right)\left(x_{1}-x_{2}\right)}= \\
& +\frac{-\left(x_{1}+x_{2}\right)\left(x_{1}-x_{2}\right) y_{0}+\left(x_{0}+x_{2}\right)\left(x_{0}-x_{2}\right) y_{1}-\left(x_{0}+x_{1}\right)\left(x_{0}-x_{1}\right) y_{2}}{\left(x_{0}-x_{1}\right)\left(x_{0}-x_{2}\right)\left(x_{1}-x_{2}\right)} x+ \\
& +\frac{x_{1} x_{2}\left(x_{1}-x_{2}\right) y_{0}-x_{0} x_{2}\left(x_{0}-x_{2}\right) y_{1}+x_{0} x_{1}\left(x_{0}-x_{1}\right) y_{2}}{\left(x_{0}-x_{1}\right)\left(x_{0}-x_{2}\right)\left(x_{1}-x_{2}\right)}= \\
& =k x^{2}+l x+m
\end{aligned}
$$

where $k, l, m$ are coefficients of the polynomial:

$$
\begin{aligned}
k & =\frac{y_{0}}{\left(x_{0}-x_{1}\right)\left(x_{0}-x_{2}\right)}+\frac{y_{1}}{\left(x_{0}-x_{1}\right)\left(x_{2}-x_{1}\right)}+\frac{y_{2}}{\left(x_{0}-x_{2}\right)\left(x_{1}-x_{2}\right)} \\
l & =-\frac{\left(x_{1}+x_{2}\right) y_{0}}{\left(x_{0}-x_{1}\right)\left(x_{0}-x_{2}\right)}-\frac{\left(x_{0}+x_{2}\right) y_{1}}{\left(x_{0}-x_{1}\right)\left(x_{2}-x_{1}\right)}-\frac{\left(x_{0}+x_{1}\right) y_{2}}{\left(x_{0}-x_{2}\right)\left(x_{1}-x_{2}\right)} \\
m & =\frac{x_{1} x_{2} y_{0}}{\left(x_{0}-x_{1}\right)\left(x_{0}-x_{2}\right)}+\frac{x_{0} x_{2} y_{1}}{\left(x_{0}-x_{1}\right)\left(x_{2}-x_{1}\right)}+\frac{x_{0} x_{1} y_{2}}{\left(x_{0}-x_{2}\right)\left(x_{1}-x_{2}\right)}
\end{aligned}
$$

Using

$$
\begin{aligned}
t_{1} & =\frac{y_{0}}{\left(x_{0}-x_{1}\right)\left(x_{0}-x_{2}\right)} \\
t_{2} & =\frac{y_{1}}{\left(x_{0}-x_{1}\right)\left(x_{2}-x_{1}\right)} \\
t_{3} & =\frac{y_{2}}{\left(x_{0}-x_{2}\right)\left(x_{1}-x_{2}\right)}
\end{aligned}
$$

equations 3.5 can be simplified:

$$
\begin{aligned}
k & =t_{1}+t_{2}+t_{3} \\
l & =-\left(x_{1}+x_{2}\right) t_{1}-\left(x_{0}+x_{2}\right) t_{2}-\left(x_{0}+x_{1}\right) t_{3} \\
m & =x_{1} x_{2} t_{1}+x_{0} x_{2} t_{2}+x_{0} x_{1} t_{3}
\end{aligned}
$$


In general case, the coefficients $k, l, m$ of the quadratic polynomial are

$$
\begin{aligned}
k & =\frac{y_{j-1}}{\left(x_{j-1}-x_{j}\right)\left(x_{j-1}-x_{j+1}\right)}+\frac{y_{j}}{\left(x_{j-1}-x_{j}\right)\left(x_{j+1}-x_{j}\right)}+\frac{y_{j+1}}{\left(x_{j-1}-x_{j+1}\right)\left(x_{j}-x_{j+1}\right)} \\
l & =-\frac{\left(x_{j}+x_{j+1}\right) y_{j-1}}{\left(x_{j-1}-x_{j}\right)\left(x_{j-1}-x_{j+1}\right)}-\frac{\left(x_{j-1}+x_{j+1}\right) y_{j}}{\left(x_{j-1}-x_{j}\right)\left(x_{j+1}-x_{j}\right)}-\frac{\left(x_{j-1}+x_{j}\right) y_{j+1}}{\left(x_{j-1}-x_{j+1}\right)\left(x_{j}-x_{j+1}\right)} \\
m & =\frac{x_{j} x_{j+1} y_{j-1}}{\left(x_{j-1}-x_{j}\right)\left(x_{j-1}-x_{j+1}\right)}+\frac{x_{j-1} x_{j+1} y_{j}}{\left(x_{j-1}-x_{j}\right)\left(x_{j+1}-x_{j}\right)}+\frac{x_{j-1} x_{j} y_{j+1}}{\left(x_{j-1}-x_{j+1}\right)\left(x_{j}-x_{j+1}\right)}
\end{aligned}
$$

The formula for integration with generalised composite Simpson's rule:

$$
\begin{aligned}
I_{n}(f) & =\sum_{j=1}^{n / 2} \int_{x_{2 j-2}}^{x_{2 j}}\left(k x^{2}+l x+m\right) d x=\sum_{j=1}^{n / 2}\left(\left.\frac{k x^{3}}{3}\right|_{x_{2 j-2}} ^{x_{2 j}}+\left.\frac{l x^{2}}{2}\right|_{x_{2 j-2}} ^{x_{2 j}}+\left.m x\right|_{x_{2 j-2}} ^{x_{2 j}}\right)= \\
& =\sum_{j=1}^{n / 2}\left(\frac{k}{3}\left(x_{2 j}^{3}-x_{2 j-2}^{3}\right)+\frac{l}{2}\left(x_{2 j}^{2}-x_{2 j-2}^{2}\right)+m\left(x_{2 j}-x_{2 j-2}\right)\right)
\end{aligned}
$$

\section{Overlapping parabolas}

The approximation of the integrand $f(x)$ with the second order polynomial $p_{2}(x)$ can be used to develop the integration rule which is based on overlapping parabolas. The rule combines an integrating and a smoothing features [9,23]. Let $a \leq x_{0}, x_{1}, \ldots, x_{n} \leq b$ be a set of unequally spaced abscissas. As was shown, using the Lagrange's formula for the interpolating polynomial it is possible to define the second order function that approximates $f(x)$ at three consecutive points $x_{j-1}, x_{j}, x_{j+1}$ for $j=1,2, \ldots, n-1$ :

$$
p_{2}\left(x_{j-1}, x_{j}, x_{j+1}\right)=k_{j} x^{2}+l_{j} x+m_{j}
$$

where coefficients $k_{j}, l_{j}, m_{j}$ can be found from equations 3.8 .

On the intervals $\left[x_{0}, x_{1}\right]$ and $\left[x_{n-1}, x_{n}\right]$ no smoothing is done and the integral is calculated using these formulae:

$$
\begin{aligned}
I_{n}^{1}(f) & =\int_{x_{0}}^{x_{1}} p_{2}\left(x_{0}, x_{1}, x_{2}\right) d x \\
I_{n}^{2}(f) & =\int_{x_{n-1}}^{x_{n}} p_{2}\left(x_{n-2}, x_{n-1}, x_{n}\right) d x
\end{aligned}
$$

On all other intervals the integral is calculated as an average integral of two overlapping 
parabolas

$$
\begin{aligned}
I_{n}^{3}(f)= & \frac{1}{2} \int_{x_{j}}^{x_{j+1}}\left(p_{2}\left(x_{j-1}, x_{j}, x_{j+1}\right)+p_{2}\left(x_{j}, x_{j+1}, x_{j+2}\right)\right) d x= \\
= & \frac{1}{2} \int_{x_{j}}^{x_{j+1}}\left(k_{j} x^{2}+l_{j} x+m_{j}+k_{j+1} x^{2}+l_{j+1} x+m_{j+1}\right) d x= \\
= & \frac{1}{2} \int_{x_{j}}^{x_{j+1}}\left(\left(k_{j}+k_{j+1}\right) x^{2}+\left(l_{j}+l_{j+1}\right) x+\left(m_{j}+m_{j+1}\right)\right) d x= \\
= & \frac{k_{j}+k_{j+1}}{2}\left(\frac{x_{j+1}^{3}-x_{j}^{3}}{3}\right)+\frac{l_{j}+l_{j+1}}{2}\left(\frac{x_{j+1}^{2}-x_{j}^{2}}{2}\right)+\frac{m_{j}+m_{j+1}}{2}\left(x_{j+1}-x_{j}\right) \\
& \quad \text { for } \quad j=1,2, \ldots, n-2
\end{aligned}
$$

The final integral value is obtained by adding the values of all three integrals. One of the advantages of this rule over the generalised composite Simpson's formula is that odd and even numbers of the integration points can be used on the integration interval.

\section{Spline integration}

Integration on unequally spaced abscissas can also be performed using the natural cubic spline approximation, presented in section 1.2 . Formula 1.45 can be used for this purpose.

\section{General remarks}

Other numerical schemes which perform integration using unequally distributed points, such as the Gauss quadrature and the Lobatto rule, are not considered here. These integration rules do not allow an arbitrary placement of the integration points. Moving a point to a specific location will require repositioning of all other points and recalculation of all information associated with the points. In addition to that, when using the Gauss quadrature, it is not possible to place the points at the limits of the integration interval.

Each of the presented integration rules places specific demands on the interval manager. The demands are related to a minimal number of the integration points required on each subinterval and to their location. At least three points are needed for the generalised composite Simpson's rule and for the spline integration on every subinterval. Four points are required to take full advantage of the integration rule based on overlapping parabolas. As for the generalised composite trapezoidal rule, due to the lower order polynomial used for the interpolation, more points must be placed at highly curved regions of the integrand profile to decrease the integration error. 


\subsection{Calculation of bending moment using adaptive noniterative strategy}

The developed adaptive noniterative strategy provides a possibility to select certain components of the interval manager and the interval processor and, thus, allows constructing specific schemes for adaptive integration. Performance of several schemes in calculating the moment resulting from bending of a beam under tension is tested in this section. The obtained bending moment $M_{\text {num }}$ is compared to the analytical one $M_{\text {analytical }}$ which is calculated using equation 2.25. The relative difference between $M_{\text {num }}$ and $M_{\text {analytical }}$ is used to quantify the error of numerical integration. Based on the calculations performed in section 2.1.5 the set of material and process parameters is chosen for which the numerical integration error is the highest. The material of the beam is IF steel (see table 2.1 for the material properties). The beam thickness is $1 \mathrm{~mm}$. The radius of bending is $5 \mathrm{~mm}$ and the calculations are performed for a range of values of tension. Reference solutions, obtained by using the traditional integration schemes with 20 integration points, are shown in figure 3.4 .

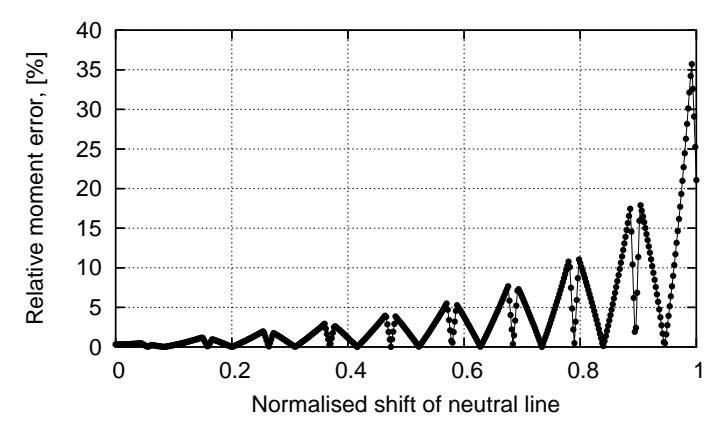

(a) Trapezoidal rule

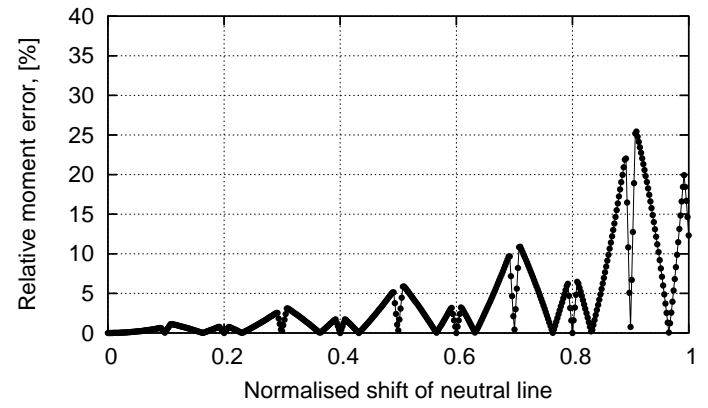

(b) Simpson's rule

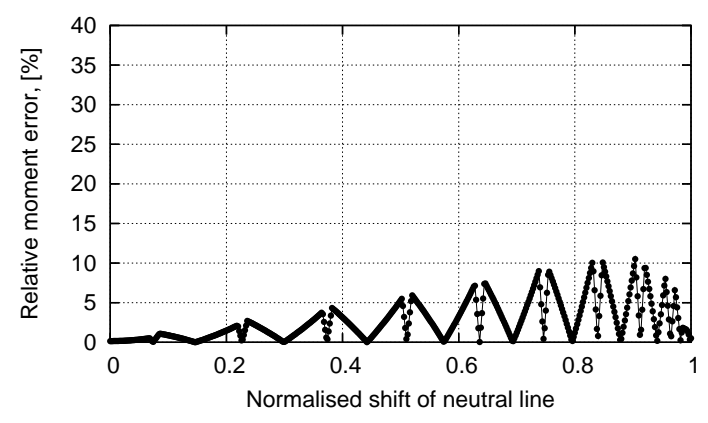

(c) Gauss quadrature

Figure 3.4: Relative moment error as a function of in-plane tension. Integration is performed with 20 i.p.s.

Comparing the graphs $3.4(\mathrm{a})$ and 2.7 it is possible to see that for a smaller number of integration points the distance between the peeks increases. Furthermore, figure 3.4(c) shows that the relative moment error obtained with the Gauss quadrature at higher tension forces decreases. This can be explained by recalling the fact that under these process 
conditions one of the points of discontinuity is reaching the outer surface and the integrand becomes most curved, like a polynomial (see figure 2.5(b)). Comparing to the trapezoidal and Simpson's rules, Gauss quadrature uses a higher order approximating polynomial that better represents the integrand under these process conditions.

\subsubsection{Adaptive scheme 1}

The interval manager of this adaptive scheme identifies the location of the points of discontinuity in the integrand profile, splits the integration interval into three subintervals and adapts location of the integration points on every subinterval. The number of the integration points on each subinterval remains constant, as shown in figure 3.5. Two or three integration points are used on the subinterval BC. The remaining points are equally distributed between the subintervals $\mathrm{AB}$ and $\mathrm{CD}$. The interval processor is allowed to use

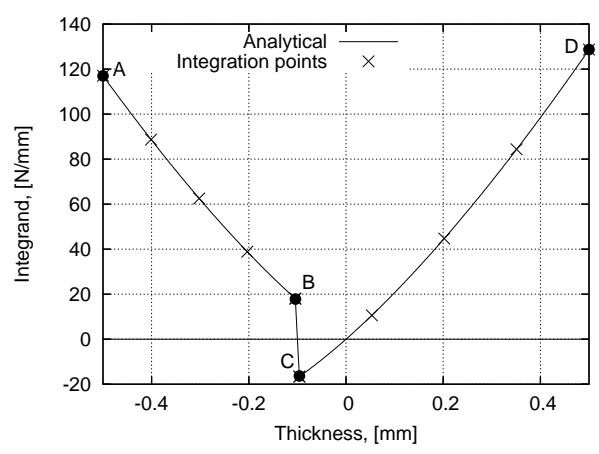

(a) Equally distributed points

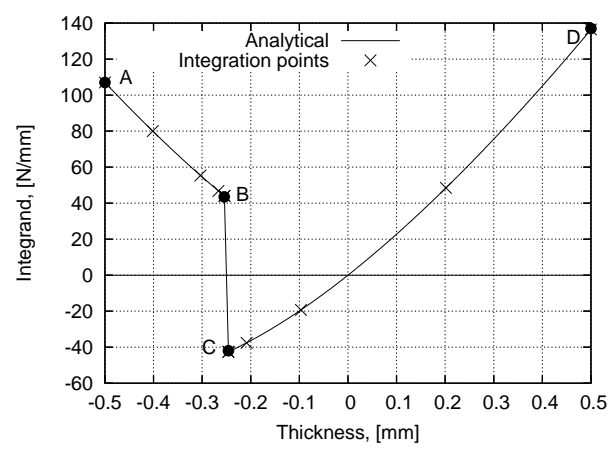

(b) Unequally distributed points

Figure 3.5: Distribution of 10 points on integration interval.

any of the integration rules with unequally distributed points.

Results of calculations with the generalised composite trapezoidal rule are shown in figure 3.6. These calculations are performed with different total number of the integration points. All integration points are equally spaced. It can be seen that tracing the location of points

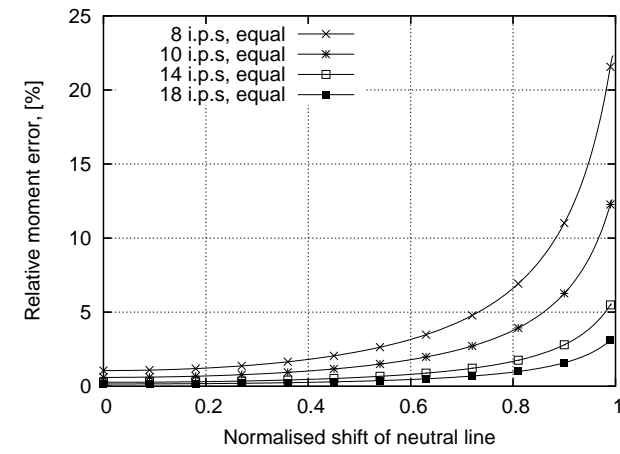

Figure 3.6: Adaptive scheme 1, trapezoidal rule.

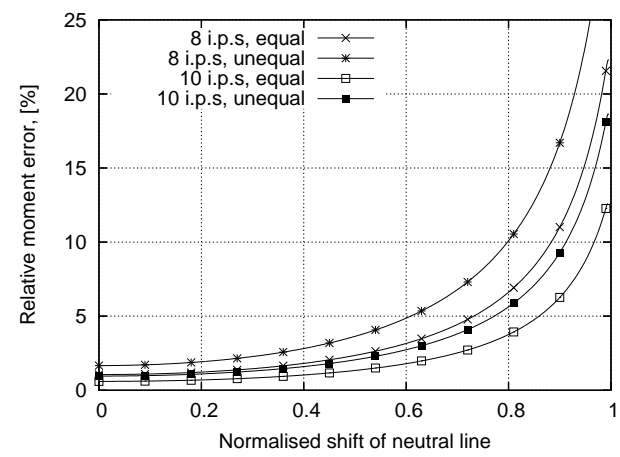

Figure 3.7: Adaptive scheme 1, trapezoidal rule. 
of discontinuity helps to get rid of the error oscillation. Furthermore, as can be seen in figure 3.8 the reasonable level of the integration error is obtained while using twice as less integration points. The relative moment error increases smoothly with increasing the tension. As was explained earlier, it increases due to the decreasing value of the bending moment and the increasing non-smoothness of the integrand.

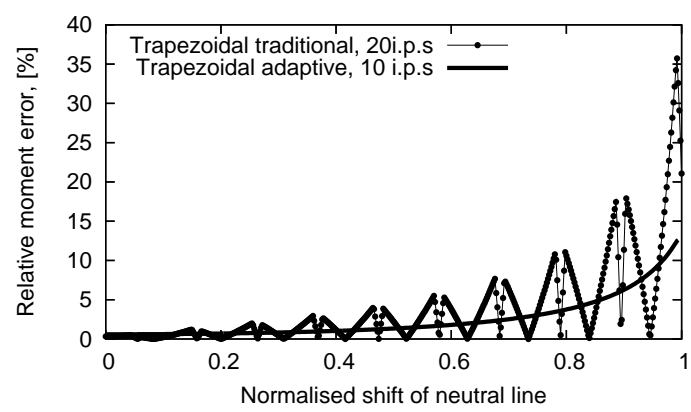

Figure 3.8: Advantages of adaptive integration.

An additional set of calculations is performed to see if the error can be decreased further by densifying the integration points in the regions with high integrand's curvature. The total number of the integration points on every subinterval remains unchanged and their concentration is increased near the points $\mathrm{B}$ and $\mathrm{C}$ in the integrand's profile (see figure $3.5(\mathrm{~b}))$. The results, presented in figure 3.7, show that the integration error increases contrary to expectations. However, it can be explained by recalling the fact that one of the subintervals (subinterval CD in figure 3.5(b)) increases in size with increasing the tension. Therefore, the contribution to the integration error from this particular interval will become higher if the total number of the integration points does not change. The integration error will increase even further since, due to the shifting, less points are used in the major part of the subinterval.

Results of calculations when the spline integration is used by the interval processor are shown in figures 3.9 and 3.10 . The advantages of using a smooth and a flexible function for the integrand approximation are clearly visible. Comparing to the generalised composite trapezoidal rule much less integration points are needed to reach the low level of the integration error. Additionally, it can be seen from figure 3.10 that shifting the integration points towards the regions with high integrand's curvature decreases the error even further. Placing more points in the highly curved regions gives the spline which better approximates the integrand and, the integration error decreases.

Outstanding results are obtained when the interval processor uses the generalised composite Simpson's rule and the rule based on overlapping parabolas, as shown in figures 3.11 and 3.12. The negligible integration error is obtained with only 7 points. The Simpson's rule outperforms the spline integration and the rule based on overlapping parabolas. Despite using less accurate approximating function the accuracy of the Simpson's rule is higher due to the fortuitous cancellation of errors (see figure 1.2). Since the integration error is already small, less than $2.5 \%$, no attempt is made to decrease it further by using the unequally distributed points. 


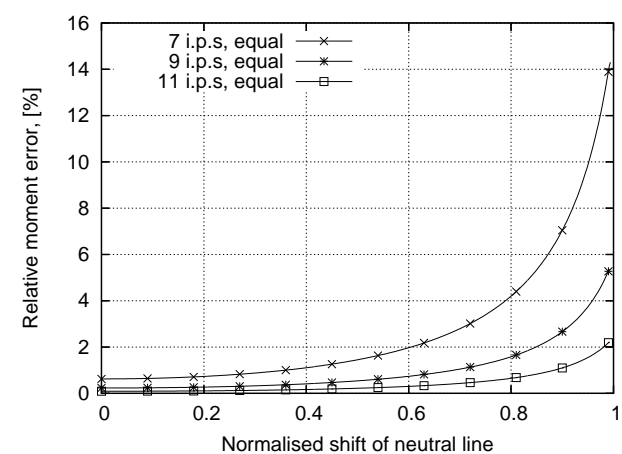

Figure 3.9: Adaptive scheme 1, spline integration.

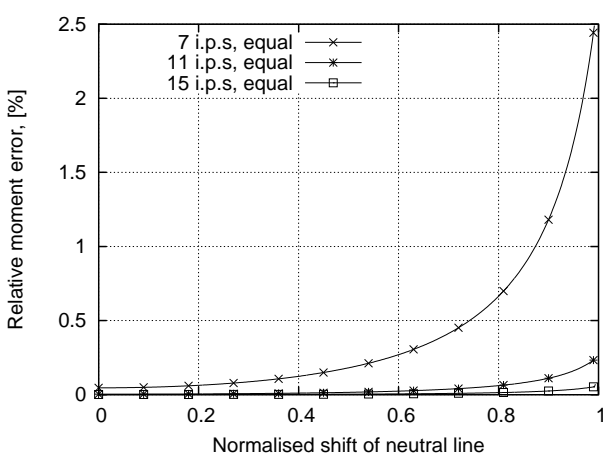

Figure 3.11: Simpson's rule.

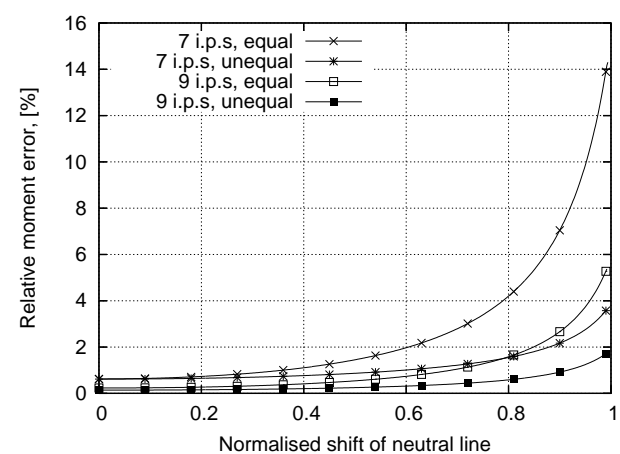

Figure 3.10: Adaptive scheme 1, spline integration.

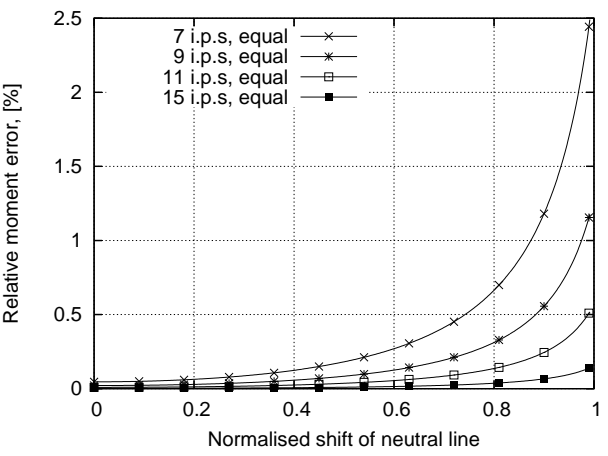

Figure 3.12: Overlapping parabolas.

\subsubsection{Adaptive scheme 2}

Most of the tasks, performed by the interval manager of the second adaptive integration scheme, are similar to the previous one. It identifies location of the discontinuities, splits the integration interval into three subintervals and adapts location of integration points on every subinterval. However, in contrast to the first adaptive scheme, the number of the integration points on every subinterval changes as a function of its length. It is known that with increasing the tension the subinterval $A B$ decreases in size. Therefore, it is logical to move the excessive integration points from this subinterval to that which increases in size (the subinterval CD in figure 3.13). Depending on a problem, there can be plenty of conditions for transferring the integration points. Here, if the length of the subinterval $A B$ becomes less than $60 \%$ of its initial value, an integration point is transferred to the increasing subinterval. The accuracy of the scheme in which the interval processor uses the generalised composite trapezoidal rule increases considerably. Figure 3.14 shows results of calculations with 8 and 10 integration points which are equally spaced on every subinterval. The previous results of the calculations with 8,10 and 14 points are added to this plot for comparison. They are marked as 8 i.p. scheme 1, 10 i.p. scheme 1 and 14 i.p. scheme 1 correspondingly. It can be seen, that as soon as an integration point is transferred to the increasing subinterval the integration error decreases abruptly. The error becomes even lower when using 10 integration points. As shown in figure 3.14(b) several relocations 


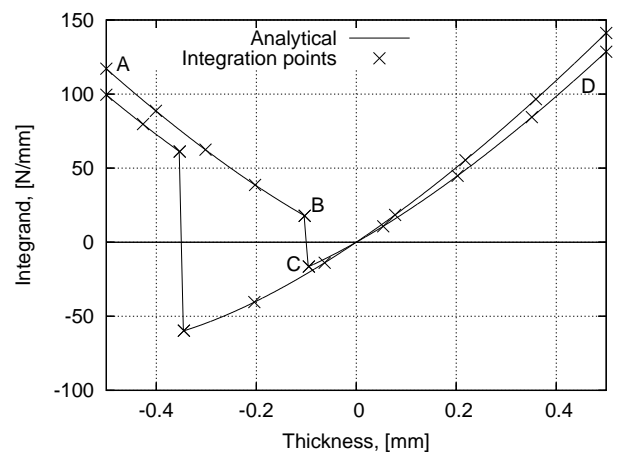

Figure 3.13: Distribution of 10 points on integration interval for various values of tension.

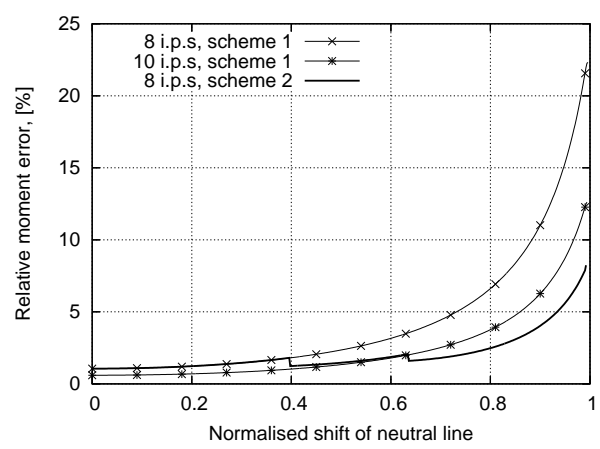

(a) 8 integration points

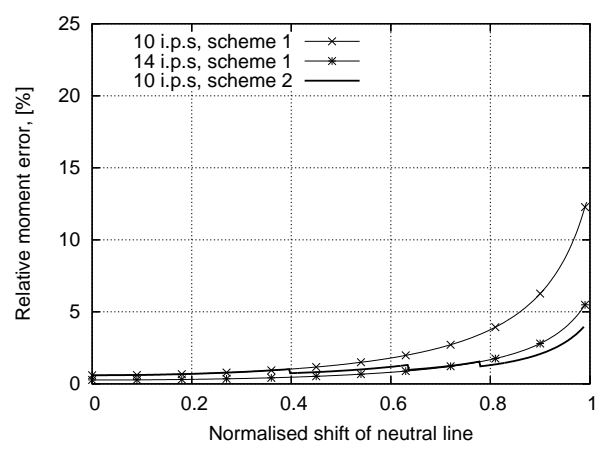

(b) 10 integration points

Figure 3.14: Comparison of performance of adaptive schemes. Interval processor uses the trapezoidal rule.

of the integration points take place and the error due to numerical integration becomes less than 4\%. Similar calculations are performed with the spline integration. 9 and 10

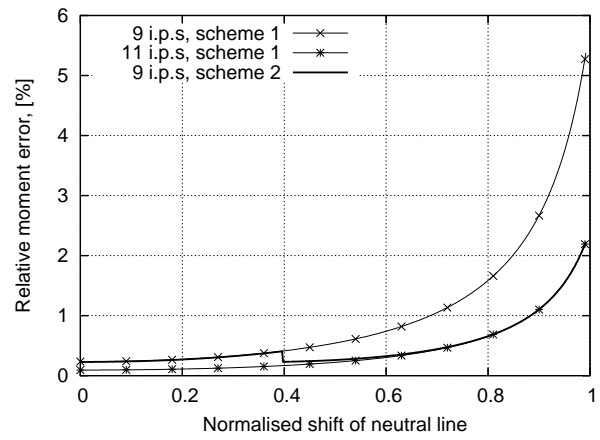

(a) 9 integration points

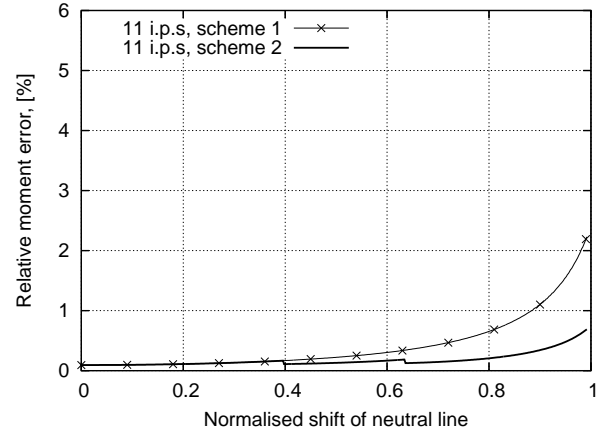

(b) 11 integration points

Figure 3.15: Adaptive scheme 2. Spline integration.

integration points are used and results are presented in figure 3.15. From figure 3.15(a) it is interesting to note that after transferring a point from the subinterval $A B$ to the subinterval $C D$ the error follows the curve obtained from the rule 11 i.p.s, scheme 1. This 
rule uses 11 integration points in total and therefore 5 points on the subinterval $C D$. This fact shows that when discontinuities are accounted for, the increasing subinterval $C D$ has the biggest contribution to the total integration error and must be properly described.

Finally, the redistribution of the integration points is employed together with the generalised composite Simpson's rule and the overlapping parabolas. The total number of the integration points is 11. Two points must be moved at the same time for the Simpson's rule to keep their number uneven on every subinterval. The obtained integration error is shown in figures 3.16 and 3.17 .

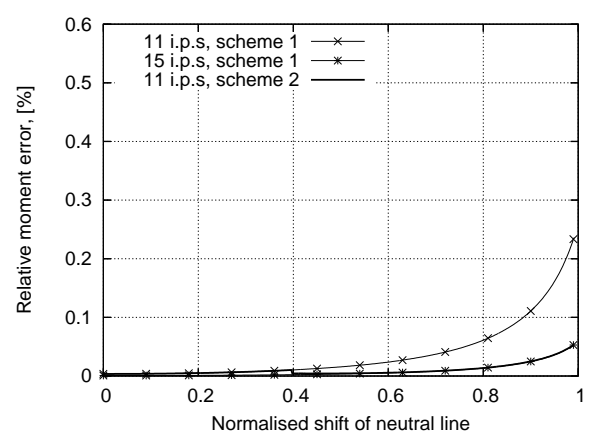

Figure 3.16: Simpson's rule.

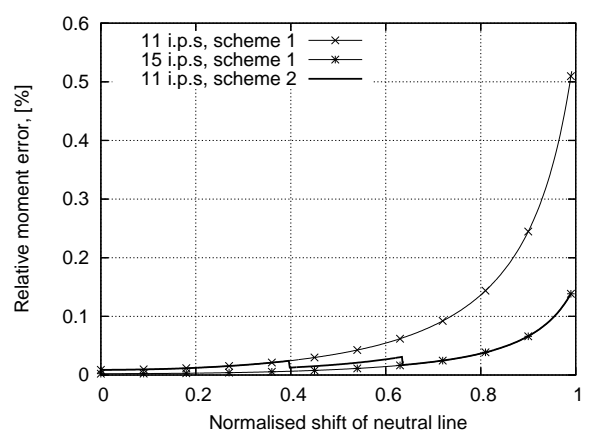

Figure 3.17: Overlapping parabolas.

\subsubsection{Summary of results}

Advantages of the adaptive noniterative strategy over the traditional integration schemes are demonstrated in this section. Two adaptive schemes tuned specifically to the bending with tension problem were developed and tested. As shown in figures 3.18 and 3.19, both schemes perform well and the negligible integration error is obtained while using a limited number of the integration points. When using an adaptive scheme, by tracing the location

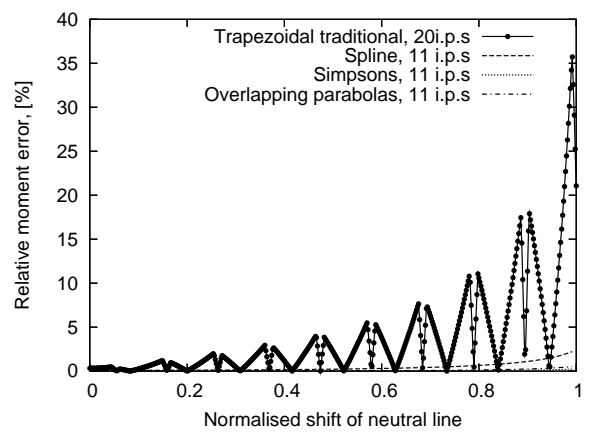

Figure 3.18: Adaptive scheme 1.

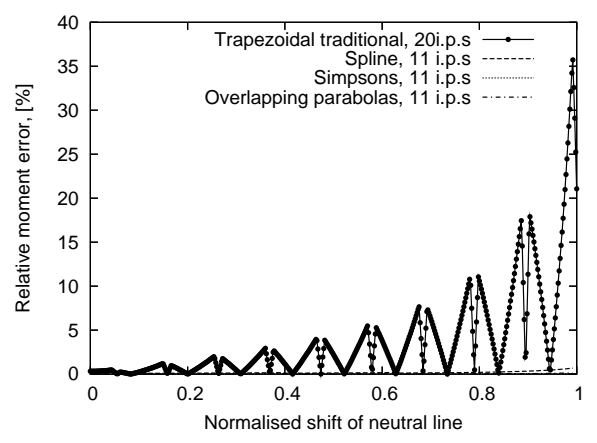

Figure 3.19: Adaptive scheme 2 .

of the points of discontinuity, the error oscillation can be suppressed and as a result, the integration error increases smoothly with increasing the tension. The best performance the lowest integration error while using the smallest number of points - can be achieved when the Simpson's rule is used by the interval processor. 


\section{Chapter 4}

\section{Adaptive integration strategy for Kirchhoff triangular elements}

In this chapter the adaptive noniterative integration strategy is developed further to make it applicable to a three-dimensional finite element analysis with shell elements. Some modifications of the interval manager are discussed and several new components are introduced. Since the strategy is developed for the Kirchhoff triangular element a brief description of this element type is provided.

\subsection{Overview of integration strategy}

An implicit finite element solution procedure is considered which is often used for simulation of sheet metal forming and especially in a springback analysis. In the implicit procedure, state variables that satisfy the equilibrium in the end of a load increment are found iteratively by the Newton-Raphson method. One iteration of the Newton-Raphson procedure can generally be split into three parts: calculation of the stiffness matrix, calculation of the incremental displacement vector and calculation of the internal force vector.

Adapting the integration points during a numerical solution process will have an influence on the internal force vector value and therefore may result into a divergence of the solution process. To minimise the divergence, the adaptation of the integration points must take place after finding the converged solution in the end of the load increment. It is schematically shown in figure 4.1.

As can be seen, right after finding the converged solution the interval manager of the adaptive strategy is called, the discontinuities are located and the integration points are adapted to decrease the integration error. As soon as an integration point is relocated or newly introduced there is no history information available at this point. Therefore, the interval manager must include an extra component to calculate new values of internal variables, such as stress and strain vectors and hardening parameters.

If loading continues, the stiffness matrix is calculated and the iterative process is initiated to find state variables for the new load increment. If unloading occurs, the adapted integration 


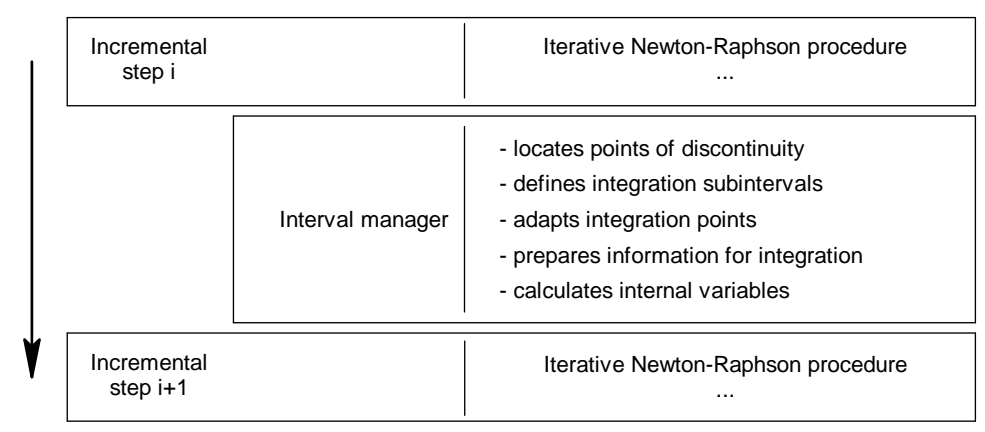

Figure 4.1: Adaptive integration within implicit finite element solution procedure.

points will guarantee more accurate stress resultants and, therefore, more accurate change of shape. It is believed that a deviation of the bending moment after adaptation from that obtained by the Newton-Raphson process is small and an influence of this difference on the change of shape can be neglected. At the same time, if loading continues the difference will be accounted for during the next load increment.

\subsection{Discrete Kirchhoff triangular element}

In simulations of sheet metal forming shell elements are commonly used. They are based on different plate bending theories which are applicable to situations when the material thickness is considerably smaller comparing to other dimensions. The theories assume that while bending a plate, plane stress state occurs and the plate geometry can be described by using variables of the middle plane. The Kirchhoff plate bending theory prohibits a transverse shear deformation which means that any straight line normal to the middle plane remains straight and normal after a deformation. Only the main definitions are introduced in this section and the complete description of the element based on the Kirchhoff theory can be found in $[1,24]$.

A deformation of a plate can be divided into a membrane part and a bending part. If one considers the membrane part, displacements of the plate can be defined using values of the middle plane [24]:

$$
\left\{\begin{array}{c}
u \\
v \\
w
\end{array}\right\}=\left\{\begin{array}{c}
u^{m p}(x, y) \\
v^{m p}(x, y) \\
w^{m p}(x, y)
\end{array}\right\}
$$

Where $u, v$ and $w$ are the displacements in $x, y$ and $z$ directions correspondingly and superscript $m p$ denotes that the displacements of the middle plane are considered. The membrane strain vector can be found from:

$$
\left\{\begin{array}{c}
\varepsilon_{x x} \\
\varepsilon_{y y} \\
\varepsilon_{z z} \\
\gamma_{x y}
\end{array}\right\}=\left\{\begin{array}{c}
\frac{\partial u}{\partial x} \\
\frac{\partial v}{\partial y} \\
\frac{\partial w}{\partial z} \\
\frac{\partial u}{\partial y}+\frac{\partial v}{\partial x}
\end{array}\right\}=\left\{\begin{array}{c}
u,_{x}^{m p} \\
v,_{y}^{m p} \\
f\left(\varepsilon_{x x}, \varepsilon_{y y}\right) \\
u,_{y}^{m p}+v,_{x}^{m p}
\end{array}\right\}
$$


The thickness-direction strain $\varepsilon_{z z}$ cannot be found from the kinematic relations. For elastic deformations it is found using constitutive relations and the in-plane components. For example, for isotropic elastic material and in case of the plane stress state the thicknessdirection strain becomes:

$$
\varepsilon_{z z}=-\frac{\nu}{1-\nu}\left(\varepsilon_{x x}+\varepsilon_{y y}\right)
$$

In case of plastic deformations the thickness strain is found from the incompressibility condition:

$$
\varepsilon_{z z}=-\varepsilon_{x x}-\varepsilon_{y y}
$$

When considering only the bending part of deformations, the vector of the plate displacements can be written as follows:

$$
\left\{\begin{array}{c}
u \\
v \\
w
\end{array}\right\}=\left\{\begin{array}{c}
0 \\
0 \\
w^{m p}(x, y)
\end{array}\right\}+z \cdot\left\{\begin{array}{c}
\theta_{y} \\
-\theta_{x} \\
0
\end{array}\right\}
$$

Variables $\theta_{x}$ and $\theta_{y}$ are rotations about $x$ and $y$ axes. The vector of strains due to the bending deformation [24]:

$$
\left\{\begin{array}{l}
\varepsilon_{x x} \\
\varepsilon_{y y} \\
\varepsilon_{z z} \\
\gamma_{x y} \\
\gamma_{x z} \\
\gamma_{y z}
\end{array}\right\}=\left\{\begin{array}{c}
\frac{\partial u}{\partial x} \\
\frac{\partial v}{\partial y} \\
\frac{\partial w}{\partial z} \\
\frac{\partial u}{\partial y}+\frac{\partial v}{\partial x} \\
\frac{\partial u}{\partial z}+\frac{\partial w}{\partial x} \\
\frac{\partial v}{\partial z}+\frac{\partial w}{\partial y}
\end{array}\right\}=\left\{\begin{array}{c}
0 \\
0 \\
f\left(\varepsilon_{x x}, \varepsilon_{y y}\right) \\
0 \\
w, m p+\theta_{y} \\
w, m p-\theta_{x}
\end{array}\right\}+z \cdot\left\{\begin{array}{c}
\theta_{y, x} \\
-\theta_{x, y} \\
0 \\
\theta_{y, y}-\theta_{x, x} \\
0 \\
0
\end{array}\right\}
$$

Combining equations 4.2 and 4.6 one obtains the complete vector of strains for the plate deformed under combined influence of bending and tension:

$$
\left\{\begin{array}{c}
\varepsilon_{x x} \\
\varepsilon_{y y} \\
\varepsilon_{z z} \\
\gamma_{x y} \\
\gamma_{x z} \\
\gamma_{y z}
\end{array}\right\}=\left\{\begin{array}{c}
u,_{x}^{m p} \\
v,_{y}^{m p} \\
f\left(\varepsilon_{x x}, \varepsilon_{y y}\right) \\
u,_{y}^{m p}+v,{ }_{x}^{m p} \\
w,_{x}^{m p}+\theta_{y} \\
w,_{y}^{m p}-\theta_{x}
\end{array}\right\}+z \cdot\left\{\begin{array}{c}
\theta_{y, x} \\
-\theta_{x, y} \\
0 \\
\theta_{y, y}-\theta_{x, x} \\
0 \\
0
\end{array}\right\}
$$

For the Kirchhoff plate bending theory the transverse shear strains must be equal zero. As a result:

$$
\left\{\begin{array}{c}
\gamma_{x z} \\
\gamma_{y z}
\end{array}\right\}=\left\{\begin{array}{c}
w,{ }_{x}^{m p}+\theta_{y} \\
w,{ }_{y}^{m p}-\theta_{x}
\end{array}\right\}=\left\{\begin{array}{l}
0 \\
0
\end{array}\right\} \Rightarrow\left\{\begin{array}{c}
\theta_{y} \\
\theta_{x}
\end{array}\right\}=\left\{\begin{array}{c}
-w,{ }_{x}^{m p} \\
w,,_{y}^{m p}
\end{array}\right\}
$$


Taking into account the Kirchhoff constraints 4.8 the complete vector of strains becomes:

$$
\left\{\begin{array}{c}
\varepsilon_{x x} \\
\varepsilon_{y y} \\
\varepsilon_{z z} \\
\gamma_{x y} \\
\gamma_{x z} \\
\gamma_{y z}
\end{array}\right\}=\left\{\begin{array}{c}
u,_{x}^{m p} \\
v,_{y}^{m p} \\
f\left(\varepsilon_{x x}, \varepsilon_{y y}\right) \\
u,_{y}^{m p}+v,{ }_{x}^{m p} \\
0 \\
0
\end{array}\right\}+z \cdot\left\{\begin{array}{c}
\theta_{y, x} \\
-\theta_{x, y} \\
0 \\
\theta_{y, y}-\theta_{x, x} \\
0 \\
0
\end{array}\right\}=\left\{\begin{array}{c}
u,{ }_{x}^{m p} \\
v,{ }_{y}^{m p} \\
f\left(\varepsilon_{x x}, \varepsilon_{y y}\right) \\
u,_{y}^{m p}+v,_{x}^{m p} \\
0 \\
0
\end{array}\right\}+z \cdot\left\{\begin{array}{c}
-w_{, x x}^{m p} \\
-w_{, y y}^{m p} \\
0 \\
-2 w_{, x y}^{m p} \\
0 \\
0
\end{array}\right\}
$$

Or in a more convenient form:

$$
\{\varepsilon\}=\{\eta\}+z \cdot\{\kappa\}
$$

In practice, the discrete Kirchhoff elements are used, in which the transverse shear strains are not equal zero throughout the element but only at specified locations - at nodes situated in the middle of an element side. The typical discrete Kirchhoff triangular element with some of its external degrees of freedom is shown in figure 4.2. In total, there are 18 degrees of freedom: 9 translational, 6 in-plane rotational and 3 out-of-plane rotational. The outof-plane rotational degrees of freedom are commonly used to prevent zero energy modes.

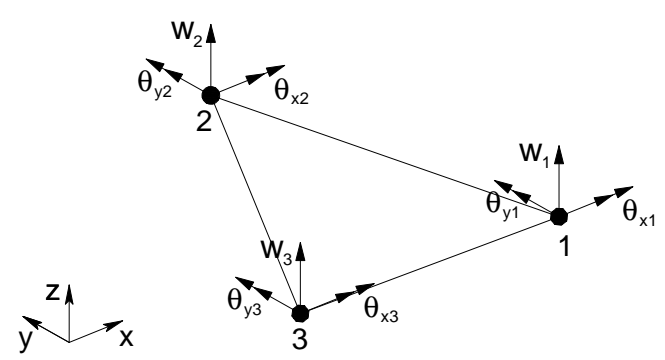

Figure 4.2: Kirchhoff triangle with some of its external degrees of freedom.

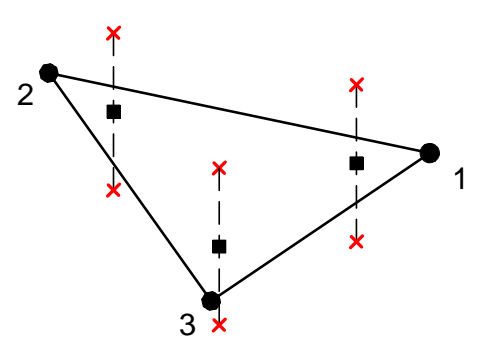

Figure 4.3: Points for in-plane and out-ofplane integration.

When using an anisotropic elastic-plastic material model, characteristic matrices of the Kirchhoff triangular element are complex volume integrals which can only be found by integrating numerically in-plane and in thickness direction. The optimal location of the in-plane integration points, indicated by squares in figure 4.3, is well-discussed in literature, see for example $[1,2,25]$. The out-of-plane integration points are indicated by crosses and the curvature values $w_{, x x}, w_{, y y}$ and $w_{, x y}$ are different for every column of them. This follows from the definition of the Kirchhoff triangular element which requires that the membrane part of the total strain $\eta$ is constant throughout the element and the bending part $\kappa$ varies linearly. As a result, for a general case of deformation, the location of the elastic-plastic transitions may be different for every column of the integration points.

\subsection{Components of interval manager}

Most of the components of the interval manager of the adaptive strategy for shell elements are comparable to those for the bending with tension problem. Differences are related to 
the presence of the new component which is required to update internal variables and to a more complex algorithm to calculate points of discontinuity in stress profiles in a general three dimensional case.

\subsubsection{Calculation of points of discontinuity}

Several points of discontinuity may be present in the through-thickness stress profile when a material undergoes cyclic bending and unbending while passing, for example, a die radius or a drawbead. Figures 4.4 and 4.6 show through-thickness stress profiles which occur in a beam after bending, elastic unbending, reverse bending and repeated bending in the original direction. The cyclic deformation is performed without in-plane tension. An elastic, perfectly plastic material is chosen and, due to the symmetry, only a half of the stress profiles is shown.

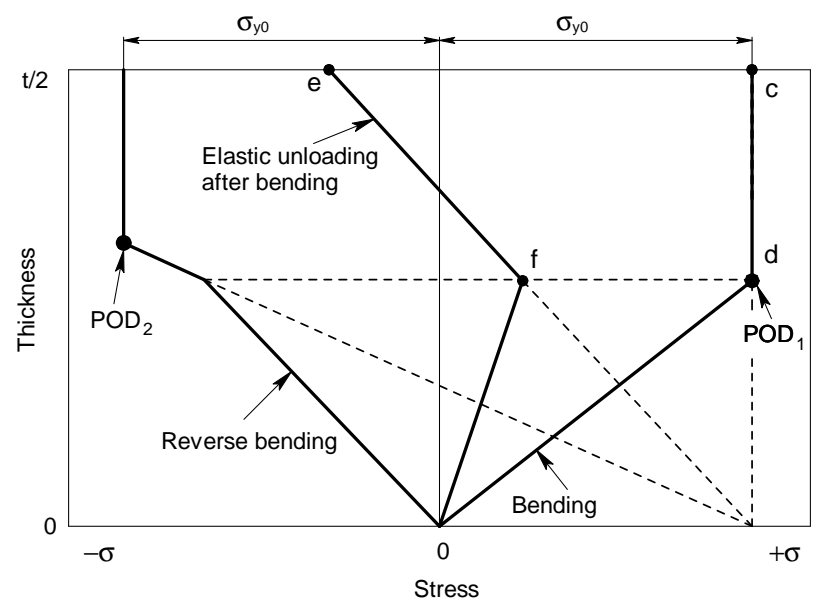

Figure 4.4: Points of discontinuity in stress profiles after bending and unbending.

While bending, as soon as yielding of the material is initiated, an elastic-plastic boundary or a point of discontinuity (POD1 in figure 4.4) appears in the stress profile. Having reached some radius of bending the material is elastically unloaded. After unloading the bilinear stress distribution remains and the upper layer of the material is in compression. For the elastic-perfectly plastic material, the yielding in the reverse direction will start after reaching the initial yield stress value $\sigma_{y 0}$. Additionally, the lines $c-d$ and $e-f$ intersect the neutral axis at the yield stress that is reached during the bending part of the cycle. To show this, the equation that describes stresses during unloading is derived. For simplicity, only one cycle consisting of bending and unbending of a beam without tension is considered. From equation 2.1 the bending strain can be approximated by:

$$
\varepsilon_{\theta}=\frac{z}{\rho}
$$

where $z$ is the through-thickness coordinate and $\rho$ is the bending radius. Equations 2.5 and 2.12 can be used to find the stress distribution in the regions of elastic and plastic 
deformations:

$$
\sigma_{\theta}^{e}=\frac{E}{1-\nu^{2}} \frac{z}{\rho}, \quad \sigma_{\theta}^{p}=\sigma_{y 0}
$$

where $\sigma_{y 0}=2 / \sqrt{3} \sigma_{u n}$ is the plane strain yield stress.

Using equation 2.25 it is always possible to find the bending moment per unit width of the beam. For the specific case, shown in figure $4.5(\mathrm{a})$, there is no elastic zone in the stress profile and the bending moment can be found from:

$$
M=\frac{\sigma_{y 0} t^{2}}{4}
$$

During an elastic unloading the circumferential stresses decrease in value. This causes the

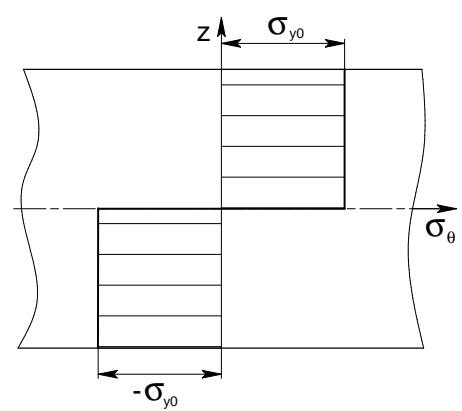

(a) bending

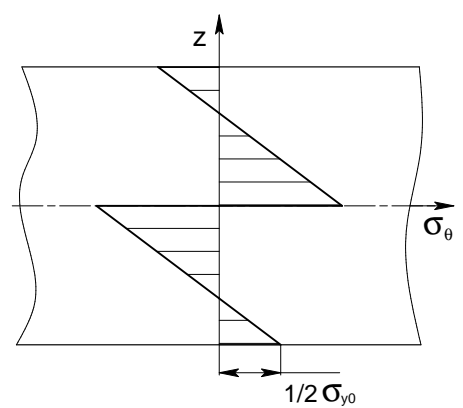

(b) elastic unloading

Figure 4.5: Through-thickness stress profiles for rigid, perfectly plastic material.

change of the bending moment which, from equation 2.35, is equal to:

$$
\Delta M=\frac{t^{3}}{12} \frac{\Delta \sigma_{\theta}}{z}
$$

For the bending moment $M=\sigma_{y 0} t^{2} / 4$ the removal of external forces during the elastic unloading results in $\Delta M=-\sigma_{y 0} t^{2} / 4$ and therefore the change of the circumferential stresses becomes:

$$
\Delta \sigma_{\theta}=-\frac{12 z}{t^{3}} M=-3 \sigma_{y 0} \frac{z}{t}
$$

Let $\sigma_{A}$ and $\sigma_{B}$ be values of the circumferential stresses during the loading and the elastic unloading. Then, the stress in a through-thickness point after the unloading can be found as follows:

$$
\sigma_{B}=\sigma_{A}+\Delta \sigma_{\theta}=\sigma_{A}-3 \sigma_{y 0} \frac{z}{t}=\sigma_{y 0}-3 \sigma_{y 0} \frac{z}{t}
$$

The through-thickness stress profile after the elastic unloading is shown in figure $4.5(\mathrm{~b})$. The line $e-f$ (see figure 4.4) can be described by using equation 4.16 and for $z=0$ this line intersects the neutral axis at the yield stress point.

Using similar considerations it is possible to define the point of intersection of the lines $c-d$ and $e-f$ for a material with linear hardening. Again, from equations 2.5 and 2.12 the stresses in the elastic and plastic regions can be written as follows:

$$
\sigma_{\theta}^{e}=E^{\prime} \frac{z}{\rho}, \quad \sigma_{\theta}^{p}=\sigma_{y 0}+E_{t}\left(\frac{z}{\rho}-\frac{\sigma_{y 0}}{E^{\prime}}\right)
$$


In this equation $E_{t}$ is the slope of the stress-strain curve in the region of plastic deformations and $E^{\prime}=E /\left(1-\nu^{2}\right)$. The point of intersection of the line $c-d$ with the neutral line can be found by substituting $z=0$ into the last equation:

$$
\sigma_{\theta}^{p}=\sigma_{y 0}-\sigma_{y 0} \frac{E_{t}}{E^{\prime}}=\sigma_{y 0}-\beta
$$

Using equation 4.15 the line $e-f$ of the stress profile can be defined by:

$$
\sigma_{B}=\sigma_{A}+\Delta \sigma_{\theta}=\sigma_{y 0}+E_{t}\left(\frac{z}{\rho}-\frac{\sigma_{y 0}}{E^{\prime}}\right)-\frac{12 z}{t^{3}} M
$$

This equation shows that if $z=0$ the lines $c-d$ and $e-f$ intersect the neutral line at the same point, namely at the point where the stress is equal to $\sigma_{y 0}-\beta$.

Yielding of the material during loading in the reverse direction produces a new elasticplastic boundary and a new point of discontinuity (POD2). If the second elastic-plastic boundary does not propagate further than the first one, the stress profile contains two points of discontinuity. Unloading the beam from this situation and plastically loading it in the original direction produces a new point of discontinuity (POD3) (see figure 4.6). The description of algorithms for calculating through-thickness location of the points of discontinuity POD1 and POD2 resulting from bending and reverse bending is given below.

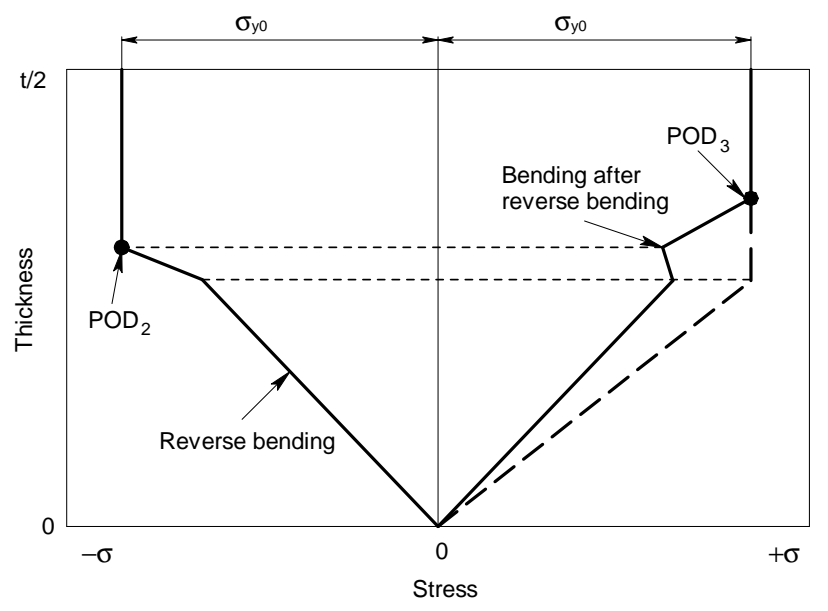

Figure 4.6: Point of discontinuity in stress profile after repeated bending in original direction.

To explain the algorithm for locating POD1, bending a strip to a certain radius is considered. The strip is deformed under a combined influence of a bending moment and tension. The typical distribution of the equivalent stress in a cross-section of the strip for a material with arbitrary hardening is shown in Figure 4.7. In this figure $\sigma_{y}$ is the stress at yield and the variable $a$ defines the shift of the neutral line which occurs due to applied tension.

To identify the location of POD1 the strategy of Armen and Pifko [7] can be followed. Let $l=z-\frac{t}{2}$ be the coordinate of the elastic-plastic transition with respect to the central line. 


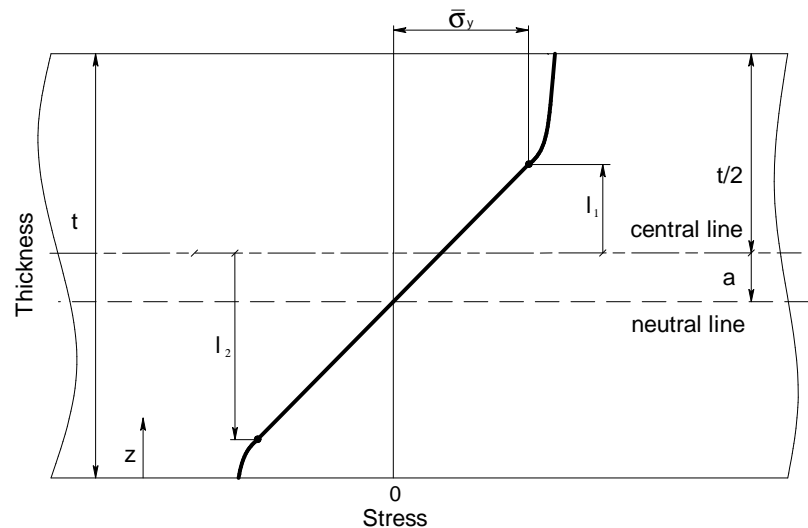

Figure 4.7: Equivalent stress profile after bending.

$\mathrm{z}$ is measured from the lower surface of the strip with: $z=0$ at the lower surface, $z=\frac{t}{2}$ at the central line, and $z=t$ at the upper surface. The vector of stresses for a material in elastic regime can be written using the Hooke's law for plane stress:

$$
\left\{\begin{array}{l}
\sigma_{x x} \\
\sigma_{y y} \\
\tau_{x y}
\end{array}\right\}=\frac{E}{1-\nu^{2}}\left[\begin{array}{ccc}
1 & \nu & 0 \\
\nu & 1 & 0 \\
0 & 0 & 1-\nu
\end{array}\right]\left\{\begin{array}{l}
\varepsilon_{x x} \\
\varepsilon_{y y} \\
\gamma_{x y}
\end{array}\right\}
$$

The complete vector of strains using the Kirchhoff theory with $\mathrm{z}$ measured from the lower surface becomes:

$$
\left\{\begin{array}{l}
\varepsilon_{x x} \\
\varepsilon_{y y} \\
\gamma_{x y}
\end{array}\right\}=\left\{\begin{array}{c}
u,_{x}^{m p} \\
v,{ }_{y}^{m p} \\
u,{ }_{y}^{m p}+v,{ }_{x}^{m p}
\end{array}\right\}-l \cdot\left\{\begin{array}{c}
w_{, x x}^{m p} \\
w_{, y y}^{m p} \\
2 w_{, x y}^{m p}
\end{array}\right\}
$$

Combining equations 4.20 and 4.21 one obtains expressions for the components of the stress vector:

$$
\begin{aligned}
\sigma_{x x} & =\frac{E}{1-\nu^{2}}\left[u_{, x}^{m p}-l w_{, x x}^{m p}+\nu\left(v_{, y}^{m p}-l w_{, y y}^{m p}\right)\right] \\
\sigma_{y y} & =\frac{E}{1-\nu^{2}}\left[v_{, y}^{m p}-l w_{, y y}^{m p}+\nu\left(u_{, x}^{m p}-l w_{, x x}^{m p}\right)\right] \\
\tau_{x y} & =\frac{E}{2(1+\nu)}\left[u_{, y}^{m p}+v_{, x}^{m p}-2 l w_{, x y}^{m p}\right]
\end{aligned}
$$

In the text that follows the superscript $m p$ will be omitted for simplicity. Equations 4.22 can also be rewritten as follows:

$$
\begin{aligned}
\sigma_{x x} & =\frac{E}{1-\nu^{2}}\left(u_{, x}+\nu v_{, y}\right)-l \frac{E}{1-\nu^{2}}\left(w_{, x x}+\nu w_{, y y}\right) \\
\sigma_{y y} & =\frac{E}{1-\nu^{2}}\left(v_{, y}+\nu u_{, x}\right)-l \frac{E}{1-\nu^{2}}\left(w_{, y y}+\nu w_{, x x}\right) \Rightarrow \\
\tau_{x y} & =\frac{E}{2(1+\nu)}\left(u_{, y}+v_{, x}\right)-l \frac{E}{1+\nu} w_{, x y}
\end{aligned}
$$




$$
\begin{aligned}
\sigma_{x x} & =N_{x x}-l M_{x x} \\
\sigma_{y y} & =N_{y y}-l M_{y y} \\
\tau_{x y} & =N_{x y}-l M_{x y}
\end{aligned} \quad \Rightarrow \quad\{\sigma\}=\{N\}+l\{M\}
$$

Where $\{N\}$ represents the membrane contribution to the stress and $\{M\}$ represents the bending contribution. It is known that a material remains elastic through its thickness until the yield condition is satisfied. A function that relates the stress state to the onset of yielding is called yield function. By substituting the components of the stress vector into a yield function equation it is possible to derive the expression for $l$.

The von Mises yield function for the three-dimensional stress state:

$$
\sqrt{\left(\sigma_{x x}-\sigma_{y y}\right)^{2}+\left(\sigma_{x x}-\sigma_{z z}\right)^{2}+\left(\sigma_{y y}-\sigma_{z z}\right)^{2}+6 \tau_{x y}^{2}+6 \tau_{x z}^{2}+6 \tau_{y z}^{2}}-\sqrt{2} \sigma_{u n}=0
$$

For the plane stress situation it reduces to:

$$
\sigma_{x x}^{2}+\sigma_{y y}^{2}-\sigma_{x x} \sigma_{y y}+3 \tau_{x y}^{2}=\sigma_{u n}^{2}
$$

Substituting the stress components from equation 4.24 into equation 4.26 and gathering terms gives:

$$
\begin{aligned}
& \left(M_{x x}^{2}+M_{y y}^{2}+3 M_{x y}^{2}-M_{x x} M_{y y}\right) l^{2}- \\
& -\left(N_{x x} M_{x x}+N_{y y} M_{y y}+3 N_{x y} M_{x y}-\frac{1}{2}\left(N_{y y} M_{x x}+N_{x x} M_{y y}\right)\right) 2 l+ \\
& +N_{x x}^{2}+N_{y y}^{2}+3 N_{x y}^{2}-N_{x x} N_{y y}=\sigma_{u n}^{2}
\end{aligned}
$$

Using additional variables $\mathrm{A}, \mathrm{B}$ and $\mathrm{C}$ :

$$
\begin{aligned}
& A=M_{x x}^{2}+M_{y y}^{2}+3 M_{x y}^{2}-M_{x x} M_{y y} \\
& B=-2\left(N_{x x} M_{x x}+N_{y y} M_{y y}+3 N_{x y} M_{x y}-\frac{1}{2}\left(N_{y y} M_{x x}+N_{x x} M_{y y}\right)\right) \\
& C=N_{x x}^{2}+N_{y y}^{2}+3 N_{x y}^{2}-N_{x x} N_{y y}
\end{aligned}
$$

equation 4.27 can be simplified to give:

$$
A l^{2}+B l+C-\sigma_{u n}^{2}=0
$$

Solving this quadratic equation gives two values of $l$ :

$$
l_{1,2}=\frac{-B \pm \sqrt{B^{2}-4 A\left(C-\sigma_{u n}^{2}\right)}}{2 A}
$$

which define the location of the elastic-plastic transitions POD1 for the case of bending with tension.

As can be seen, to locate the transition points the algorithm uses the strain vector components which are readily available after each incremental step of the finite element solution 
procedure. If, for example, Kirchhoff triangular elements are used to describe the strip, then by performing similar calculations for each column of integration points for every element in the mesh, the interval manager can always track the location of POD1 during the analysis.

To define the method for calculating location of POD2 cyclic bending of a strip under tension is considered. For simplicity, an isotropic elastic, perfectly plastic material is considered. The fictive distribution of equivalent stresses through the thickness after bending and reverse bending is shown in figure 4.8. In this figure $\bar{\sigma}_{y 1}$ is the initial yield stress and

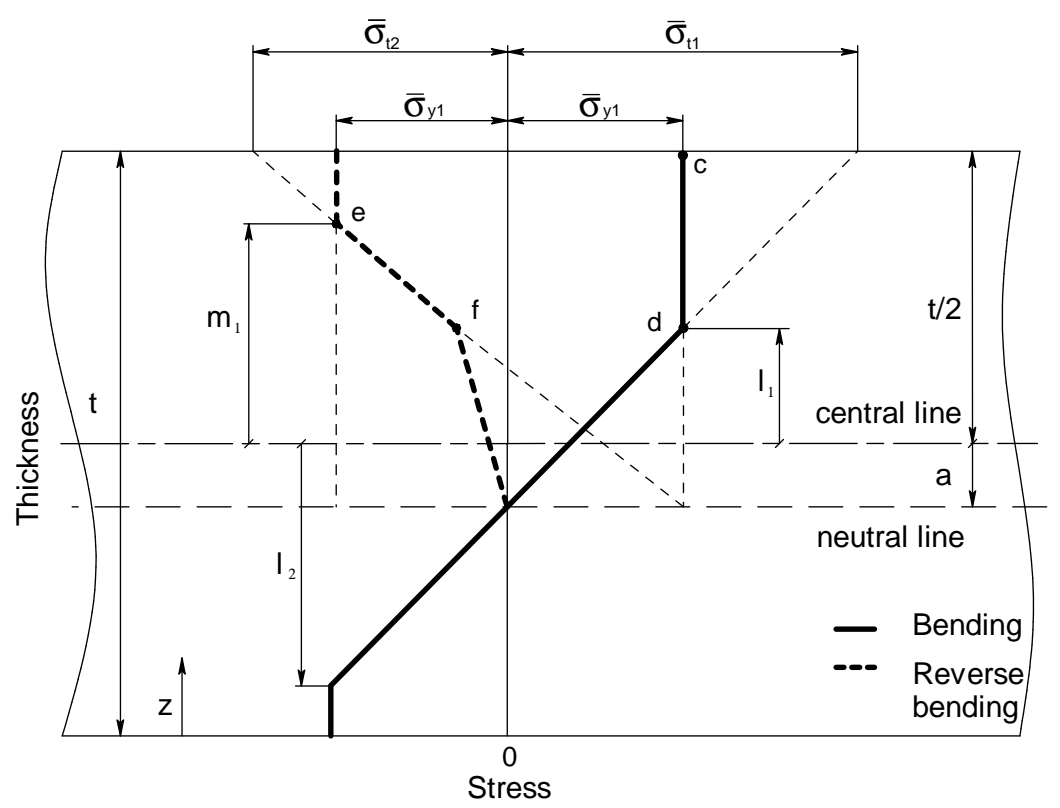

Figure 4.8: Locating point of discontinuity in stress profile for bending and reverse bending. Elastic, perfectly plastic material.

$\bar{\sigma}_{t 1}$ is the effective trial stress computed using the total strains as elastic strains. Based on the method presented above it is possible to calculate $l_{1}$ and $l_{2}$ - the coordinates of POD1 after bending. Knowing these values the neutral line shift $a$ can be found:

$$
a=l_{1}-\frac{l_{1}-l_{2}}{2}=\frac{l_{1}+l_{2}}{2}
$$

Using similar triangles it is possible to calculate the location of the elastic-plastic transition which occurs during the reverse bending [7]:

$$
\begin{gathered}
\frac{m_{1}-a}{\frac{t}{2}-a}=\frac{2 \bar{\sigma}_{y 1}}{\bar{\sigma}_{y 1}+\bar{\sigma}_{t 2}} \Rightarrow \\
m_{1}=a+\frac{2 \bar{\sigma}_{y 1}}{\bar{\sigma}_{y 1}+\bar{\sigma}_{t 2}} \cdot\left(\frac{t}{2}-a\right)
\end{gathered}
$$

where $\bar{\sigma}_{t 2}$ is the effective stress that would exist if the material did not have plastic deformations during the reverse loading. The through-thickness location of the second POD2 
can be calculated from the condition of symmetry about the neutral line:

$$
m_{2}=-\left(\left|m_{1}\right|+2|a|\right)
$$

Now the method for calculating the location of POD2 is developed further to be applicable for an isotropic elastic-plastic material with linear hardening. The fictive distribution of effective stresses through the thickness after bending and reverse bending is shown in figure 4.9. In this figure $\bar{\sigma}_{1}$ is the effective stress reached during the loading part of the cycle.

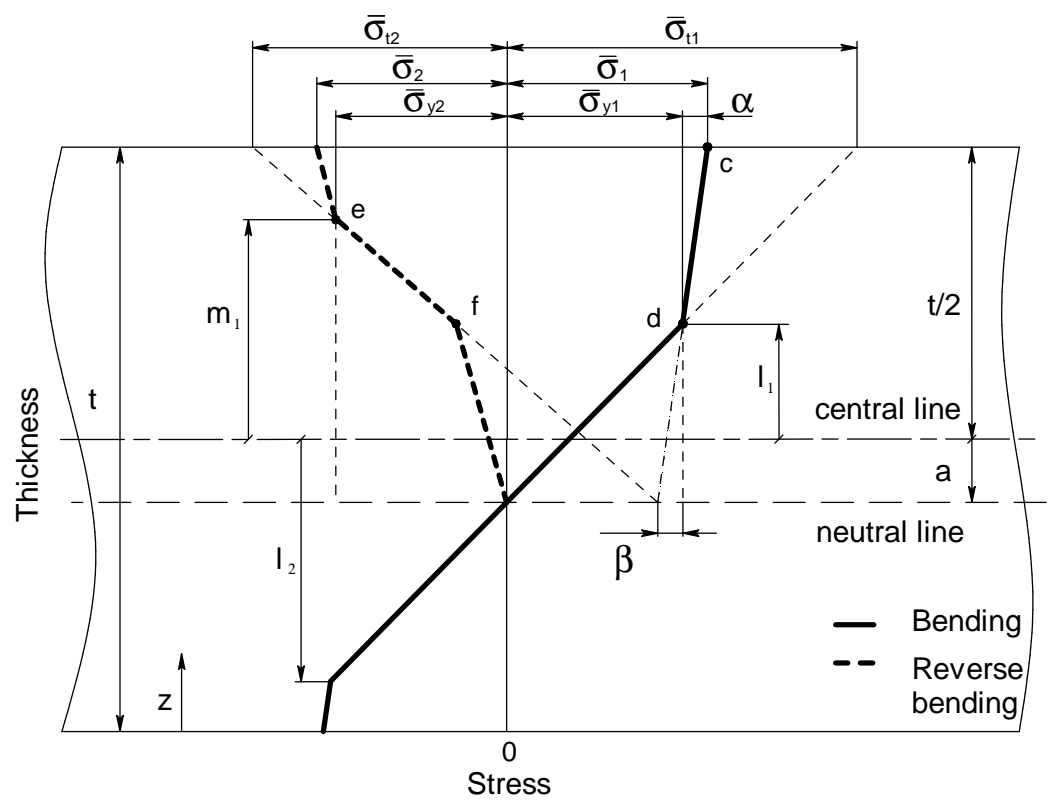

Figure 4.9: Locating point of discontinuity in stress profile for bending and reverse bending. Elastic-plastic material with linear hardening.

$\alpha=\bar{\sigma}_{1}-\bar{\sigma}_{y 1}$ can be described as a measure of the material hardening. Variable $\beta$ defines the location where the lines $c-d$ and $e-f$ intersect the neutral line and can be calculated from:

$$
\beta=\frac{l_{1}-a}{\frac{t}{2}-l_{1}} \alpha
$$

Using similar triangles it is possible to calculate the location of the elastic-plastic transition which occurs during the reverse bending [7]:

$$
\begin{gathered}
\frac{m_{1}-a}{\frac{t}{2}-a}=\left(\frac{\bar{\sigma}_{y 1}+\bar{\sigma}_{y 2}-\beta}{\bar{\sigma}_{y 1}+\bar{\sigma}_{t 2}-\beta}\right) \Rightarrow \\
m_{1}=a+\left(\frac{\bar{\sigma}_{y 1}+\bar{\sigma}_{y 2}-\beta}{\bar{\sigma}_{y 1}+\bar{\sigma}_{t 2}-\beta}\right) \cdot\left(\frac{t}{2}-a\right)
\end{gathered}
$$

where $\bar{\sigma}_{2}$ is the effective stress obtained in the upper layer during the bending in reverse direction, $\bar{\sigma}_{y 2}$ is the yield stress and $\bar{\sigma}_{t 2}$ is the effective stress that would exist if the material did not have plastic deformations during the reverse loading. The through-thickness 
location of the second POD2 can be calculated from the condition of symmetry about the neutral line.

This method can be used for the calculation of the positions of POD2 when $m_{1}>l_{1}$. Increasing the bending moment in the reverse loading will cause POD2 propagate further and as soon as it coincides with POD1 the method that calculates the location of POD1 must be used.

It can be seen that $\beta$ can only be calculated under the assumption of linear distribution of the stresses in the plastic range. This assumption may introduce some error in calculating the location of POD2. Therefore, it is required to check an extent of the error and a validity of the complete method. Additional points of discontinuity may appear in the stress profile during subsequent loading cycles (see figure 4.6) which may produce more complex, multilinear distributions of stresses and strains. As a result, an application of the strategy underlying the presented methods to define the algorithm for locating POD3 will require extra assumptions and the accuracy of the method will be questionable. An alternative strategy is needed to locate the discontinuities in stress profiles obtained during cyclic loading for a material with arbitrary hardening. A simple example of such a strategy is given in chapter 5 of this report.

\subsubsection{Update of internal variables}

Having found the location of the discontinuities in the stress profile the interval manager divides the complete integration interval $\left[-\frac{t}{2}, \frac{t}{2}\right]$ into several subintervals. Integration points are rearranged so that there are several points inside every subinterval and two of the points are lying on its limits. If required, the location and the number of the integration points on each subinterval can be adapted depending on numerical integration scheme used and smoothness of the integrand. A newly introduced or relocated integration point does not contain any information about previous loading and its internal variables must be calculated.

The $J_{2}$ flow model based on the von Mises yield surface is often used to describe plasticity in metals. The key assumption of the model is that the plastic flow in metals is unaffected by hydrostatic stresses. Only deviatoric stresses have influence on the yield condition and the plastic flow direction. The detailed formulation of the $J_{2}$ flow theory of plasticity can be found in $[1,4]$. The rate of the plastic multiplier $\dot{\lambda}$ in the von Mises theory is equal to the rate of the accumulated effective plastic strain $\dot{\bar{\varepsilon}}$. Additionally, the only hardening parameter $q$ equals the accumulated effective plastic strain $\bar{\varepsilon}$. During the iterative-incremental solution procedure, quantities that must be available at each integration point and updated as the solution progresses are stresses $\{\sigma\}$ and the hardening parameter $q$. Therefore, for every new integration point these values must be calculated.

Figure 4.10 shows distributions of the total effective strain, the effective plastic strain and the effective stress through the thickness of a strip deformed in bending with tension. For simplicity, only the region is shown where the material is in tension. Linear hardening is assumed and therefore the distribution of the effective stress is bilinear. Due to the in-plane tension the neutral line shifts through a distance of $a$. Location of the integration points 


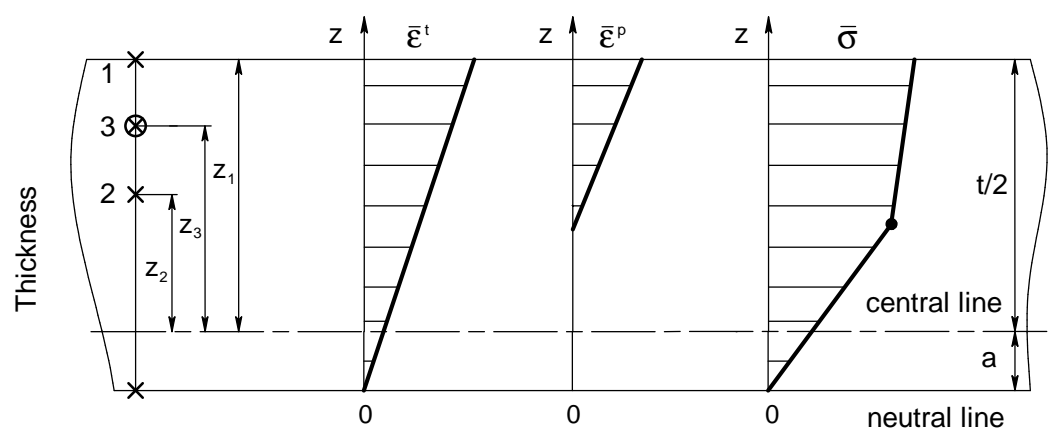

Figure 4.10: Effective stress, total strain and plastic strain distributions for bending with tension.

is also shown in this figure. Old points that were used for integration during the previous successfully converged incremental step are shown with the crosses. A new integration point defined by the interval manager is shown with the encircled cross. To calculate the internal variables for the new integration point 3 information of the old integration points 1 and 2 can be used. The linear distribution of the internal variables makes it possible to calculate values of the accumulated effective plastic strain and the effective stress by simple interpolation. From the Lagrange's formula for the interpolating polynomial:

$$
f\left(z_{3}\right)=\frac{z_{3}-z_{2}}{z_{1}-z_{2}} f\left(z_{1}\right)+\frac{z_{3}-z_{1}}{z_{2}-z_{1}} f\left(z_{2}\right)
$$

where $z_{1}, z_{2}, z_{3}$ are the through-thickness coordinates of the integration points and $f\left(z_{1}\right)$, $f\left(z_{2}\right), f\left(z_{3}\right)$ are the associated function values. Simple interpolation requires to have at least two old integration points on every region to be able to perform the interpolation of data.

The next step is to calculate components of the stress vector. The method proposed by B.K. Chun [26] can be used. To explain the method let $b$ and $c$ be ratios of the stress vector components:

$$
b=\frac{\sigma_{y y}}{\sigma_{x x}} \text { and } c=\frac{\tau_{x y}}{\sigma_{x x}}
$$

The von Mises effective stress for the plane stress situation then becomes:

$$
\begin{aligned}
\bar{\sigma} & =\sqrt{\sigma_{x x}^{2}+\sigma_{y y}^{2}-\sigma_{x x} \sigma_{y y}+3 \tau_{x y}^{2}}=\sqrt{\sigma_{x x}^{2}+b^{2} \sigma_{x x}^{2}-b \sigma_{x x}^{2}+3 c^{2} \sigma_{x x}^{2}}= \\
& =\sqrt{1+b^{2}-b+3 c^{2}} \cdot \sigma_{x x}=\rho \cdot \sigma_{x x}
\end{aligned}
$$

Using equation 4.36 it is possible to find ratios of the stress components for the integration point 3 by simple interpolation:

$$
b_{3}=f\left(b_{1}, b_{2}\right) \quad \text { and } \quad c_{3}=f\left(c_{1}, c_{2}\right)
$$

and thus the parameter $\rho$ can also be determined:

$$
\rho_{3}=\sqrt{1+b_{3}^{2}-b_{3}+3 c_{3}^{2}}
$$


Finally the components of the stress vector at the integration point 3 can be found:

$$
\sigma_{x x}=\frac{\overline{\sigma_{3}}}{\rho_{3}}, \quad \sigma_{y y}=b_{3} \sigma_{x x}, \quad \tau_{x y}=c_{3} \sigma_{x x}
$$

More physically-based algorithm for updating the stress state was defined by M. Avetisyan, see for example [27, 28]. The algorithm, which was initially developed as a part of the strategy to incorporate effects of a trimming operation in the simulation of deep drawing, is a good alternative for calculating the stress vector at the new integration point.

Through-thickness distributions of the effective strains and the effective stress which occur in a strip after bending and reverse bending under tension is shown in figure 4.11. As can be seen, after bending and reverse bending the distribution of the accumulated effective plastic strain is bilinear. Similarly to the strategy outlined above, the effective stress and

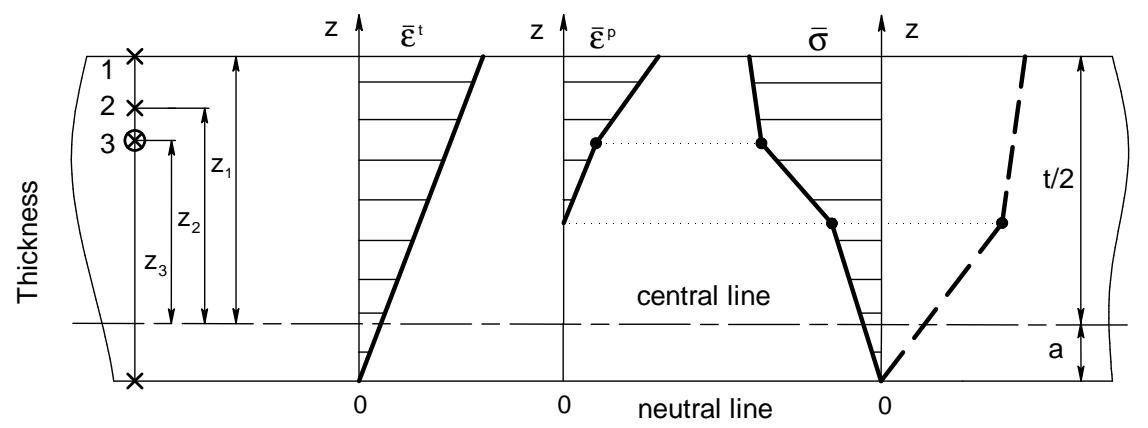

Figure 4.11: Effective stress, total strain and plastic strain distributions for bending, reverse bending with tension.

strains for the new integration point 3 can be found by simple interpolation using values of the old integration points 1 and 2 . The update of the internal variables is finished with calculating the components of the stress vector.

\subsection{Components of interval processor}

As soon as the interval manager decides on integration intervals, appropriate location of integration points and calculates information about the history of deformation, everything is available to continue the iterative-incremental finite element solution procedure. During the procedure the numerical integration is employed to find incremental strains at each integration point and to calculate the element characteristic matrices. Any of the integration rules presented in section 3.2 .2 can be used for that purpose. Application of the integration rules to the bending with tension problem revealed that the generalised composite Simpson's rule and the rule based on overlapping parabolas are superior to all others. These are simple rules that can guarantee the lowest integration error while using the smallest number of the integration points. In addition to that, comparing to the generalised composite Simpson's rule, the rule based on overlapping parabolas does not require an uneven number of the integration points on every subinterval. 


\section{Chapter 5}

\section{Alternative numerical algorithm for locating points of discontinuity}

In chapter 4 the algorithms for locating the points of discontinuity POD1 (bending) and POD2 (bending/unbending) were presented. It is known that the idea underlying these algorithms cannot be used to develop schemes for locating points of discontinuity for an arbitrary loading scenario. Already for the discontinuities POD3 (bending/unbending/bending) in addition to the linear hardening other assumptions are needed, which together with an increased complexity of the algorithm will have an impact on the accuracy of calculations [7].

\subsection{Stress profiles in realistic forming conditions}

In a realistic simulation of forming the in-plane tension may have a greater influence on through-thickness stress profiles than that which was assumed while deriving the algorithms for locating POD1 and POD2. To investigate that, several simulations of the top-hat section test are performed. The test is schematically shown in figure 5.1.

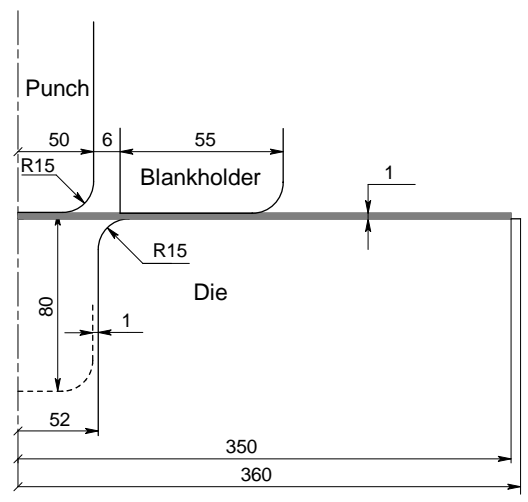

Figure 5.1: Schematic of the top-hat section test.

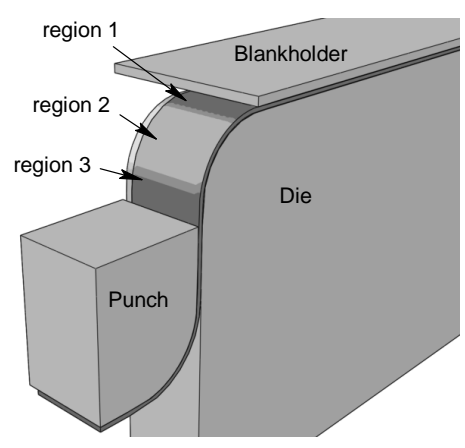

Figure 5.2: Deformation zones for data recording. 
An aluminium alloy is used in the simulations. Its properties and some process parameters are summarised in table 5.1. To describe the blank, discrete Kirchhoff triangular elements are used with 30 Gauss points through the thickness. The level of discretisation is chosen which results into 7 elements being in contact with the die radius. This mesh density minimises influence of the discretisation error on the accuracy of simulations [29]. The tools are described by means of analytical surfaces. Two simulations are performed to define the effect of the in-plane tension, namely with friction and without friction.

An element of the blank is chosen that completely passes the die radius during forming. For a column of integration points the circumferential stresses $\sigma_{x x}$ are recorded at the moment when: the element is in the bending region (region 1 in figure 5.2); the element is in the region of constant die curvature (region 2) and the element is in the unbending region (region 3 ).

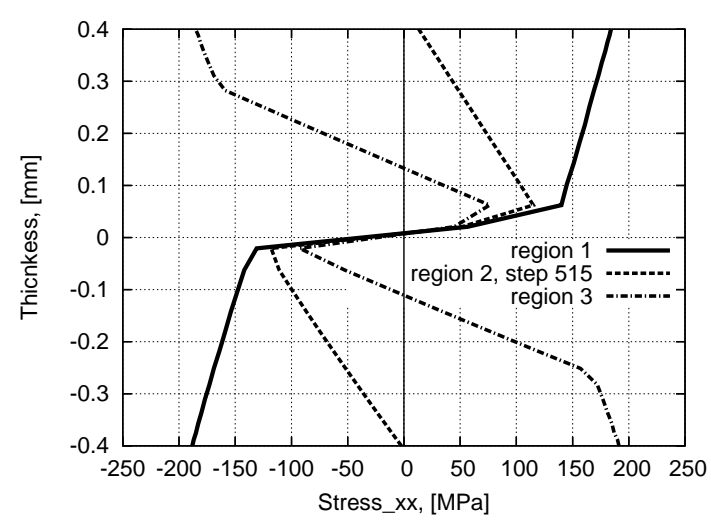

Figure 5.3: Stress profiles from simulation without friction.

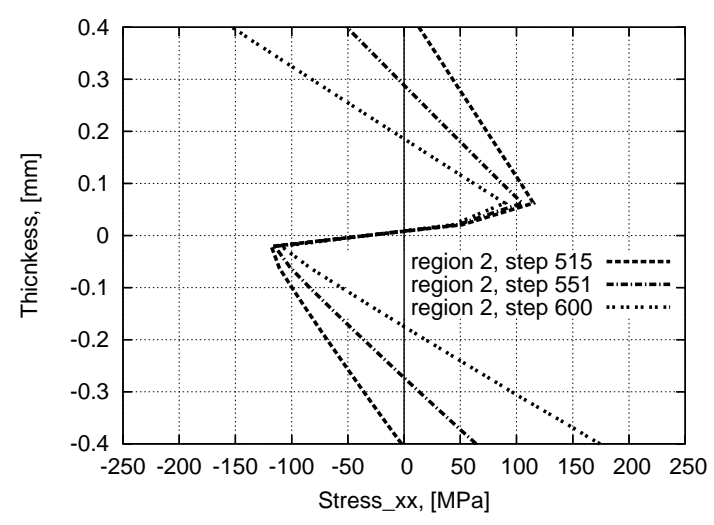

Figure 5.4: Evolution of stresses in region 2.

The simulation without friction can be characterised by negligible values of the in-plane tension. In this case, when the material enters region 1 of the die radius it is bent without tension to a certain curvature. After that, in the region of the constant die curvature loading conditions change and the material is allowed to relax. As a result, as shown in figure 5.3, the stress level decreases. The change of the stress state which takes place while

\begin{tabular}{|l|c|}
\hline Sheet thickness, $\mathrm{mm}$ & 0.81 \\
\hline Blank dimensions, $\mathrm{mm}$ & $35 \times 350$ \\
\hline Young's modulus, $\mathrm{GPa}$ & 71 \\
\hline Poisson's ratio & 0.33 \\
\hline $\mathrm{YS}_{\text {mean }}, \mathrm{MPa}$ & 135.3 \\
\hline $\mathrm{r}_{0}, \mathrm{r}_{45}, \mathrm{r}_{90}$ & $0.71,0.58,0.70$ \\
\hline $\mathrm{C}, \mathrm{MPa}$ & 576.79 \\
\hline $\mathrm{n}$ & 0.3593 \\
\hline Friction coefficient & 0.162 \\
\hline Blankholder force, $\mathrm{N}$ & 19600 \\
\hline
\end{tabular}

Table 5.1: Material and process parameters of the top-hat section test. 
moving around the die radius is shown in figure 5.4. The stress profiles at three different moments of the simulation are presented.

As soon as the material reaches the end of the die curvature it is straightened and it experiences bending in the opposite direction. It is possible that during this process the elastic limit is reached and the material starts to deform plastically, as can be seen in figure 5.3. The stress profiles shown in this figure are comparable to those considered earlier when defining the algorithms for locating the points of discontinuity POD1 and POD2 (see for example figure 4.4).

The simulation with friction shows that the in-plane tension has a significant effect on the through-thickness stress profiles. In region 1 the material is bent under tension and, as shown in figure 5.5, the shift of the neutral line takes place. While travelling around the

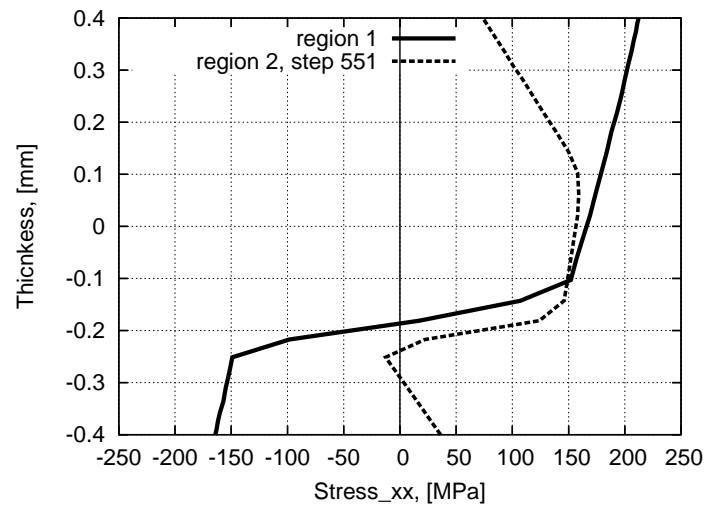

Figure 5.5: Stress profiles in regions 1 and 2.

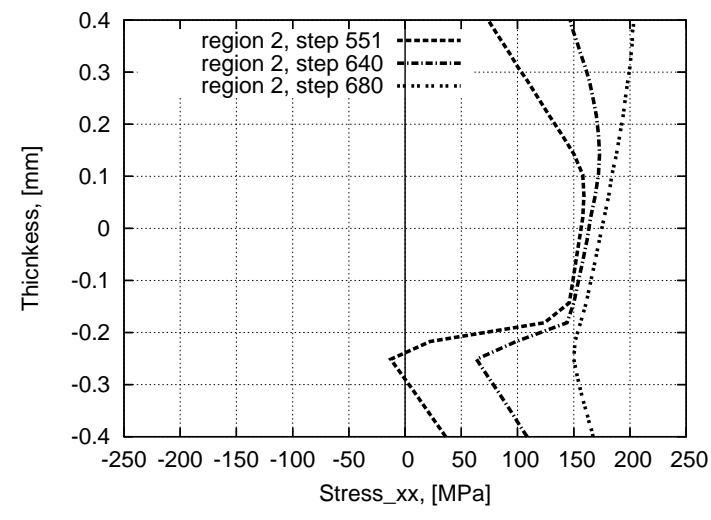

Figure 5.6: Evolution of stresses in region 2.

die radius, when the curvature does not change, the bending part of loading conditions decreases and the material mainly undergoes tensile deformations. Due to the change in loading conditions the stress relaxation occurs in the outer layers, but the main part of the material remains in tension (see figure 5.5).

Figure 5.6 shows the evolution of the stress state which occurs when the material travels around the die curvature. In this figure the stress distributions at three different moments of the simulation are plotted. It can be seen that contrary to the situation without friction the tensile deformations flatten the stress profiles and make the points of discontinuity less distinguishable. In region 3 unbending takes place and the material again experiences a combined influence of bending moment and tension. As can be seen in figure 5.7, the outer layer is in compression, the inner layer is in tension and there are two clearly visible points of discontinuity.

These results show that in realistic simulations of forming, when friction is included, the influence of tension is two-fold: it causes the shift of the neutral line and it modifies the shape of the through-thickness stress profile. The flattening which occurs in the region of constant die curvature decreases the number of points of discontinuity. The stress profiles used to locate POD2 (see for example figure 4.9) were obtained based on the assumption of uniform distribution of tension through the material thickness. In the analysis with friction this assumption is not valid and therefore the strategy to define locations of POD2, 


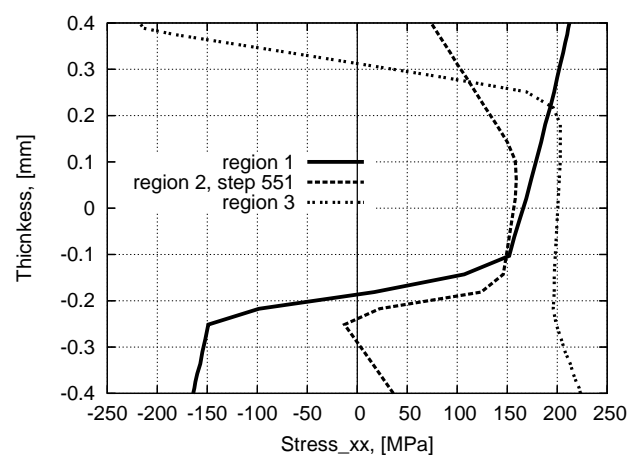

Figure 5.7: Stress profiles from simulation with friction.

presented in chapter 4, is not applicable anymore. An alternative numerical scheme is needed that is capable of locating points of discontinuity in a stress profile obtained from an arbitrary loading scenario. An idea that can be used to develop such a scheme is given below.

\subsection{Numerical scheme to locate discontinuities}

To explain the idea, pure bending of a beam to a radius of $60 \mathrm{~mm}$ is considered. Thickness of the beam is $1 \mathrm{~mm}$ and its material is an aluminium alloy. The material properties can be found in table 2.1. Using the assumptions presented in chapter 2, through-thickness stress distribution, location of elastic-plastic transitions and the resulting bending moment can be easily calculated analytically. In section 3.2 the adaptive integration strategy was defined and used to calculate the bending moment numerically. One of the components of the interval manager used the analytical formulae to define the position of the elasticplastic transitions. The main goal now is to redefine this component and to substitute the analytical formulae by a numerical procedure.

Due to the symmetry only half of the beam thickness is considered. For simplicity of explanation, there are only 4 equally spaced integration points and the interval processor of the adaptive scheme uses the generalised composite trapezoidal rule to perform the actual integration. Let the beam be deformed in two incremental steps. During the first increment the beam is bent to a radius $\rho_{1}=100 \mathrm{~mm}$. The stress distribution calculated analytically is shown in figure 5.8 .

The procedure to find the location where the material transits from elastic into plastic consists of several steps:

1) the incremental circumferential strain is calculated for every integration point. It can be found using equation 2.1:

$$
\Delta \varepsilon=\varepsilon_{1}-\varepsilon_{0}=\frac{z}{\rho_{1}}
$$




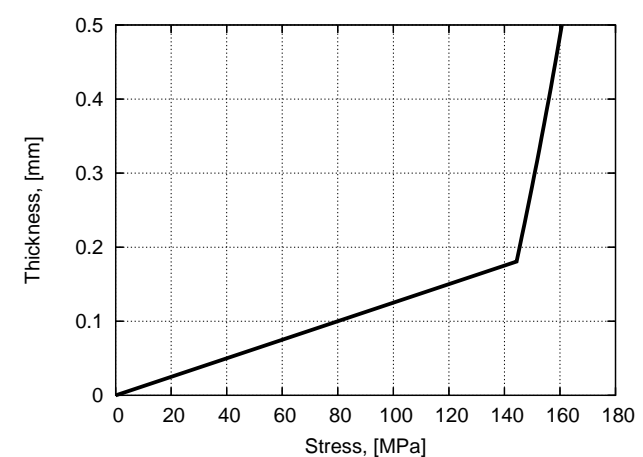

Figure 5.8: Analytical stress distribution after the first load increment.

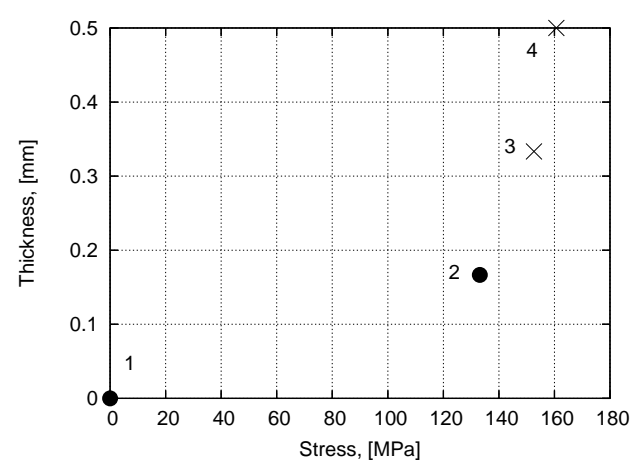

Figure 5.9: Stress values at integration points after the first load increment.

where $\varepsilon_{0}$ is the initial total strain, $\varepsilon_{1}$ is the total strain after bending and $z$ is the through-thickness coordinate. Before the bending the initial total strain equals zero, therefore:

$$
\Delta \varepsilon=\frac{z}{\rho_{1}}
$$

2) the circumferential stress is calculated for every integration point. Depending on the material and process conditions a point may be in the region of elastic or plastic deformations. As a result, different formulae must be used to find values of the circumferential stresses. To identify that, the yield condition is checked at every integration point and for this simple problem it can be written as follows [1]:

$$
F=\left|\sigma_{0}+\sigma_{\text {trial }}\right|-\sigma_{y 0}
$$

Where $\sigma_{0}$ is the circumferential stress before the deformation $\left(\sigma_{0}=0\right), \sigma_{\text {trial }}$ is the trial stress and $\sigma_{y 0}$ is the initial yield stress. The trial stress is calculated by using an assumption that the total strain increment is elastic:

$$
\sigma_{\text {trial }}=\frac{E}{1-\nu^{2}} \cdot \Delta \varepsilon=\frac{E}{1-\nu^{2}} \cdot \frac{z}{\rho_{1}}
$$

The initial yield stress $\sigma_{y 0}$, as was defined in chapter 2 , is equal to $2 / \sqrt{3} \sigma_{u n}$, where $\sigma_{u n}$ is the uniaxial yield stress. A material point is in the elastic regime if the stress state gives $F<0$ and yielding of the material occurs when $F=0$. If an integration point is elastic its value of the circumferential stress equals $\sigma_{\text {trial }}$. For integration points in the plastic region the stress is calculated using equation 2.12. The crosses, that are shown in figure 5.9, highlight the location and the associated stress values of the integration points in the plastic region (points 3 and 4). The points in the elastic region ( 1 and 2 ) are shown with the circles and, in addition to that, they are flagged as elastic until the end of the incremental loading procedure.

3) two interpolating polynomials are defined to pass through the elastic and plastic integration points. The Lagrange's formulae for the interpolating polynomial 1.6 can 
be used for this purpose. For example, the following equation defines two lines that pass through the points 1,2 and 3,4 :

$$
\begin{array}{r}
P_{1}^{j}(z)=\frac{z-z_{i+1}}{z_{i}-z_{i+1}} f\left(z_{i}\right)+\frac{z-z_{i}}{z_{i+1}-z_{i}} f\left(z_{i+1}\right) \\
\text { for } j=1,2 \text { and } i=1,3
\end{array}
$$

where $f(z)$ are the stress values at the integration points. The intersection of the lines will give an approximate location of the elastic-plastic transition (see figure 5.10).

The elastic integration point which is closest to the elastic-plastic transition is relocated. As shown in figure 5.11 it is placed to lie at the position of the stress profile discontinuity. Depending on the definition of the interval manager components, other integration points can also be relocated to better describe the stress profile.

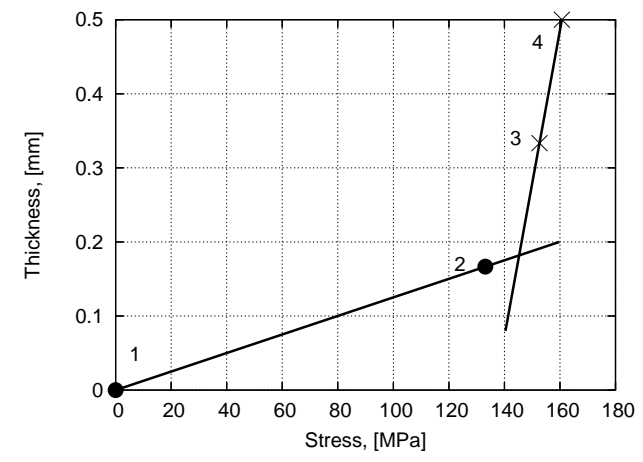

Figure 5.10: Approximate location of elastic-plastic transition point.

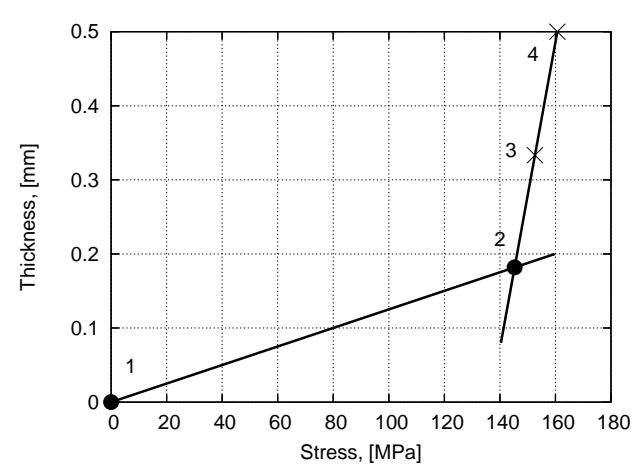

Figure 5.11: Relocation of elastic integration point.

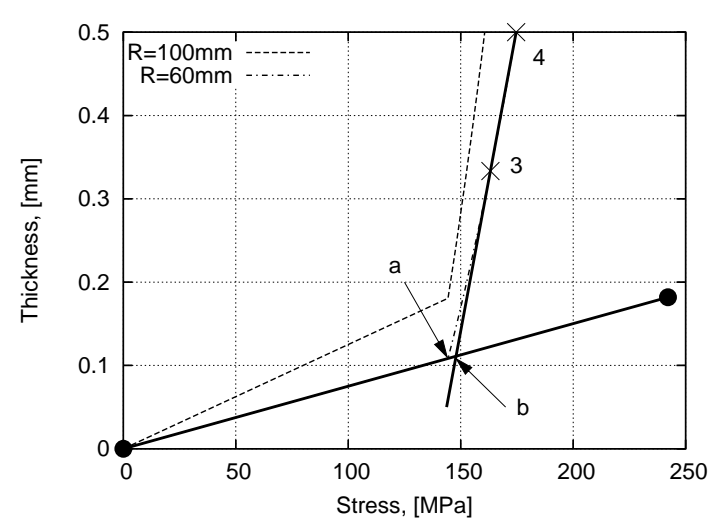

Figure 5.12: Approximate location of transition point during the second load increment. Results of analytical calculations are shown with dashed curves.

During the second load increment the beam is bent to the final radius $\rho_{2}=60 \mathrm{~mm}$. The new increment of the total strain and the new stress value are calculated for every integration point. For the points that are flagged as elastic only the trial stresses are calculated, which 
gives the accurate value of the slope in the elastic region. Two lines are again fitted to pass through the elastic and plastic points and their intersection will approximately define the new location of the elastic-plastic transition (see figure 5.12). From this figure it can be seen that there is a mismatch between the analytical and numerical points of discontinuity. However, it is believed that the difference between the points $a$ and $b$ can be reduced by using more incremental steps to bend the beam to the final radius.

It has been shown that the alternative algorithm for locating points of discontinuity in one dimensional stress profile performs well. This idea can be used as a basis to develop other more complex numerical algorithms to locate stress profile discontinuities for an arbitrary loading scenario. 



\section{Conclusions}

The error due to numerical integration in thickness direction is one of the reasons of common inaccuracy of springback prediction in a finite element analysis of sheet metal forming. During a finite element solution procedure well-known numerical schemes, such as, trapezoidal, Simpson's, Lobatto rules or Gauss quadrature are commonly used for the through-thickness integration in shell elements. For a linear analysis, when the material is in the elastic regime, any of the schemes can be used and the negligible numerical integration error can be obtained with a limited number of integration points. The situation changes when the material undergoes elastic-plastic deformations. The traditional integration rules can only guarantee low integration error when using a significant number of the integration points. Even for a simple problem up to 50 integration points may be needed to decrease the influence of numerical integration on the accuracy of springback prediction.

Influence of material and process parameters on the numerical integration error was tested using the bending with tension problem. It was shown that the error increases with increasing the sharpness of the integrand's profile near the points of discontinuity. Varying material and process parameters changes the integrand's smoothness and, as a result, traditional integration rules may require different number of the integration points to achieve the specified level of accuracy. In addition to that, when using a fixed number of the integration points their location relative to the points of discontinuity changes with varying process conditions. At some instant the location of the integration points is favourable to the numerical integration and the error is low. At some other moment, when the process conditions are slightly different, the points location is less appropriate and the integration error is high. Such oscillation makes it impossible to develop any practical guidelines that can be used to choose the number of the integration points in thickness direction. Very high number of the integration points, needed to assure certain accuracy, and the error oscillation reveal inefficiency of the traditional numerical schemes used for the through-thickness integration in shell elements.

The adaptive integration strategy for the Kirchhoff shell elements, developed in this report, may be a good alternative. It consists of two groups of components: interval manager components and interval processor components. In the end of every successfully converged incremental step of the implicit finite element solution procedure the interval manager evaluates the integrand's profile. Based on this information the location and the number of the integration points is adapted. This ensures the correct bending moment which will govern a change of shape in case of unloading. Within an incremental step, to minimise divergence of the iterative Newton-Raphson process, the integration points are not adapted 
and the interval processor is called to perform numerical integration when it is required. Presented in this report, generalised composite trapezoidal or Simpson's rules, rules based on overlapping parabolas and spline interpolation are the numerical schemes which can be used by the interval processor. For high flexibility they are all based on the integration using unequally distributed points.

Excellent results were obtained while testing the performance of the adaptive integration strategy. Calculations of the bending with tension problem showed that for a similar set of material and process parameters the adaptive Simpson's integration with 7 integration points performs significantly better than the traditional trapezoidal rule with 50 integration points. Tracing the location of discontinuities in the integrand's profile suppressed the error oscillation. As a result, for this problem it became possible to guarantee a level of the integration error which can be obtained when using the adaptive strategy with the specific number of the integration points.

Clear definition of the components of the adaptive integration strategy allows initiation of its trial implementation in finite elements. The strategy will be implemented for the Kirchhoff triangular elements in the implicit finite element code DiekA. Subsequently its performance will be tested using a number of representative examples.

It has been shown that, in its current definition, the adaptive through-thickness integration strategy for shell elements has some limitations. It only allows tracing a location of the stress profile discontinuities which occur after bending and bending/unbending and it is only applicable in situations when friction is not included into a finite element analysis. To be generally applicable, some components of the interval manager of the strategy will have to be modified. New algorithms will be developed to locate stress profile discontinuities which occur in arbitrary loading conditions. 


\section{Acknowledgements}

This project (MC1.02121) is funded by the Netherlands Institute for Metals Research. The scientific support of the project members and the financial support of the Netherlands Institute for Metals Research are greatly acknowledged. 



\section{Appendix A: Alternative formula for cubic spline.}

Let $h_{j}=x_{j+1}-x_{j}$ for $j=0,1, \ldots, n-1$. To derive a more convenient formula for the cubic spline the equation 1.34 is modified to give:

$$
\begin{aligned}
S(x) & =\frac{\left(x_{j}+h_{j}-x\right)^{3}}{6 h_{j}} M_{j}+\frac{\left(x-x_{j}\right)^{3}}{6 h_{j}} M_{j+1}+\left(y_{j}-\frac{h_{j}^{2} M_{j}}{6}\right) \frac{\left(x_{j}+h_{j}-x\right)}{h_{j}}+ \\
& +\left(y_{j+1}-\frac{h_{j}^{2} M_{j+1}}{6}\right) \frac{\left(x-x_{j}\right)}{h_{j}}=-\frac{\left(x-x_{j}-h_{j}\right)^{3}}{6 h_{j}} M_{j}+\frac{\left(x-x_{j}\right)^{3}}{6 h_{j}} M_{j+1}- \\
& -\left(y_{j}-\frac{h_{j}^{2} M_{j}}{6}\right) \frac{\left(x-x_{j}-h_{j}\right)}{h_{j}}+\left(y_{j+1}-\frac{h_{j}^{2} M_{j+1}}{6}\right) \frac{\left(x-x_{j}\right)}{h_{j}}= \\
& =-\frac{\left(x-x_{j}\right)^{3}-3\left(x-x_{j}\right)^{2} h_{j}+3\left(x-x_{j}\right) h_{j}^{2}-h_{j}^{3}}{6 h_{j}} M_{j}+\frac{\left(x-x_{j}\right)^{3}}{6 h_{j}} M_{j+1}- \\
& -\frac{x-x_{j}}{h_{j}}\left(y_{j}-\frac{h_{j}^{2} M_{j}}{6}\right)+y_{j}-\frac{h_{j}^{2} M_{j}}{6}+\frac{x-x_{j}}{h_{j}}\left(y_{j+1}-\frac{h_{j}^{2} M_{j+1}}{6}\right)= \\
& =-\frac{M_{j}}{6 h_{j}}\left(x-x_{j}\right)^{3}+\frac{M_{j}}{2}\left(x-x_{j}\right)^{2}-\frac{h_{j} M_{j}}{2}\left(x-x_{j}\right)+\frac{h_{j}^{2} M_{j}}{6}+ \\
& +\frac{M_{j+1}}{6 h_{j}}\left(x-x_{j}\right)^{3}-\frac{1}{h_{j}}\left(y_{j}-\frac{h_{j}^{2} M_{j}}{6}\right)\left(x-x_{j}\right)+y_{j}-\frac{h_{j}^{2} M_{j}}{6}+ \\
& +\frac{1}{h_{j}}\left(y_{j+1}-\frac{h_{j}^{2} M_{j+1}}{6}\right)\left(x-x_{j}\right)=\left(\frac{M_{j+1}-M_{j}}{6 h_{j}}\right)\left(x-x_{j}\right)^{3}+ \\
& +\frac{M_{j}}{2}\left(x-x_{j}\right)^{2}+\left(\frac{y_{j+1}}{h_{j}}-\frac{h_{j} M_{j+1}}{6}-\frac{h_{j} M_{j}}{2}-\frac{y_{j}}{h_{j}}+\frac{h_{j} M_{j}}{6}\right)\left(x-x_{j}\right)+y_{j}= \\
& =y_{j}+\left(\frac{y_{j+1}-y_{j}}{h_{j}}-\frac{h_{j} M_{j+1}+2 h_{j} M_{j}}{6}\right)\left(x-x_{j}\right)+\frac{M_{j}}{2}\left(x-x_{j}\right)^{2}+ \\
& +\left(\frac{M_{j+1}-M_{j}}{6 h_{j}}\right)\left(x-x_{j}\right)^{3}
\end{aligned}
$$


Choosing

$$
\begin{aligned}
c_{j} & =\frac{M_{j}}{2} \\
a_{j} & =y_{j} \\
b_{j} & =\frac{a_{j+1}-a_{j}}{h_{j}}-\frac{\left(2 c_{j}+c_{j+1}\right)}{3} h_{j}, \text { for } j=0,1, \ldots, n-1 \\
d_{j} & =\frac{c_{j+1}-c_{j}}{3 h_{j}}
\end{aligned}
$$

the cubic spline equation becomes:

$$
\begin{aligned}
S_{j}(x)= & a_{j}+b_{j}\left(x-x_{j}\right)+c_{j}\left(x-x_{j}\right)^{2}+d_{j}\left(x-x_{j}\right)^{3} \\
& \text { for } \quad x_{j} \leq x \leq x_{j+1}, \quad j=0,1, \cdots, n-1
\end{aligned}
$$




\section{Appendix B: Derivation of formula for spline integration.}

To derive the formula for spline integration a natural cubic spline $S_{j}(x)$ on interval $\Delta_{j}=$ $\left[x_{j-1}, x_{j}\right]$ can be considered. Let $h_{j}=x_{j}-x_{j-1}$. Using equation 1.34 and formally integrating the spline on the interval gives:

$$
\begin{aligned}
\int_{x_{j-1}}^{x_{j}} S_{j}(x) d x & =\int_{x_{j-1}}^{x_{j}}\left[\frac{\left(x_{j}-x\right)^{3}}{6 h_{j}} M_{j-1}+\frac{\left(x-x_{j-1}\right)^{3}}{6 h_{j}} M_{j}+\left(y_{j-1}-\frac{M_{j-1} h_{j}^{2}}{6}\right) \times\right. \\
& \left.\times \frac{\left(x_{j}-x\right)}{h_{j}}+\left(y_{j}-\frac{M_{j} h_{j}^{2}}{6}\right) \frac{\left(x-x_{j-1}\right)}{h_{j}}\right] d x
\end{aligned}
$$

To simplify the derivation the integral B-1 can be split into several parts:

$$
\int_{x_{j-1}}^{x_{j}} S_{j}(x) d x=I_{1}+I_{2}+I_{3}+I_{4}
$$

where

$$
\begin{aligned}
I_{1} & =\int_{x_{j-1}}^{x_{j}} \frac{\left(x_{j}-x\right)^{3}}{6 h_{j}} M_{j-1} d x, \\
I_{2} & =\int_{x_{j-1}}^{x_{j}} \frac{\left(x-x_{j-1}\right)^{3}}{6 h_{j}} M_{j} d x, \\
I_{3} & =\int_{x_{j-1}}^{x_{j}}\left(y_{j-1}-\frac{M_{j-1} h_{j}^{2}}{6}\right) \frac{\left(x_{j}-x\right)}{h_{j}} d x, \\
I_{4} & =\int_{x_{j-1}}^{x_{j}}\left(y_{j}-\frac{M_{j} h_{j}^{2}}{6}\right) \frac{\left(x-x_{j-1}\right)}{h_{j}} d x
\end{aligned}
$$

To solve the integral $I_{1}$ let $a=x_{j}-x$ be a new integration variable. Therefore $d a=-d x$ and the new limits of integration are $a_{l}=x_{j}-x_{j-1}=h_{j}$ and $a_{u}=0$. Then

$$
I_{1}=-\frac{M_{j-1}}{6 h_{j}} \int_{a_{l}}^{a_{u}} a^{3} d a=-\left.\frac{M_{j-1}}{6 h_{j}} \cdot \frac{a^{4}}{4}\right|_{h_{j}} ^{0}=\frac{M_{j-1} h_{j}^{3}}{24}
$$

Similarly, let $a=x-x_{j-1}$, then $d a=d x, a_{l}=0, a_{u}=h_{j}$ and the integral $I_{2}$ becomes:

$$
I_{2}=\frac{M_{j}}{6 h_{j}} \int_{a_{l}}^{a_{u}} a^{3} d a=\left.\frac{M_{j}}{6 h_{j}} \cdot \frac{a^{4}}{4}\right|_{0} ^{h_{j}}=\frac{M_{j} h_{j}^{3}}{24}
$$


The integral $I_{3}$ can be found as follows:

$$
\begin{aligned}
I_{3} & =\int_{x_{j-1}}^{x_{j}}\left(y_{j-1}-\frac{M_{j-1} h_{j}^{2}}{6}\right) \frac{\left(x_{j}-x\right)}{h_{j}} d x=\left(\frac{y_{j-1}}{h_{j}}-\frac{M_{j-1} h_{j}}{6}\right) \int_{x_{j-1}}^{x_{j}}\left(x_{j}-x\right) d x= \\
& =\left(\frac{y_{j-1}}{h_{j}}-\frac{M_{j-1} h_{j}}{6}\right)\left(\left.x_{j} x\right|_{x_{j-1}} ^{x_{j}}-\left.\frac{x^{2}}{2}\right|_{x_{j-1}} ^{x_{j}}\right)= \\
& =\left(\frac{y_{j-1}}{h_{j}}-\frac{M_{j-1} h_{j}}{6}\right)\left(x_{j}\left(x_{j}-x_{j-1}\right)-\frac{x_{j}^{2}}{2}+\frac{x_{j-1}^{2}}{2}\right)= \\
& =\left(\frac{y_{j-1}}{h_{j}}-\frac{M_{j-1} h_{j}}{6}\right)\left(x_{j}^{2}-x_{j} x_{j-1}-\frac{x_{j}^{2}-x_{j-1}^{2}}{2}\right)= \\
& =\left(\frac{y_{j-1}}{h_{j}}-\frac{M_{j-1} h_{j}}{6}\right)\left(\frac{x_{j}^{2}-2 x_{j} x_{j-1}+x_{j-1}^{2}}{2}\right)=\left(\frac{y_{j-1}}{h_{j}}-\frac{M_{j-1} h_{j}}{6}\right) \frac{\left(x_{j}-x_{j-1}\right)^{2}}{2}= \\
& =\left(\frac{y_{j-1}}{h_{j}}-\frac{M_{j-1} h_{j}}{6}\right) \cdot \frac{h_{j}^{2}}{2}=\frac{y_{j-1} h_{j}}{2}-\frac{M_{j-1} h_{j}^{3}}{12}
\end{aligned}
$$

The integral $I_{4}$ becomes:

$$
\begin{aligned}
I_{4} & =\int_{x_{j-1}}^{x_{j}}\left(y_{j}-\frac{M_{j} h_{j}^{2}}{6}\right) \frac{\left(x-x_{j-1}\right)}{h_{j}} d x=\left(\frac{y_{j}}{h_{j}}-\frac{M_{j} h_{j}}{6}\right) \int_{x_{j-1}}^{x_{j}}\left(x-x_{j-1}\right) d x= \\
& =\left(\frac{y_{j}}{h_{j}}-\frac{M_{j} h_{j}}{6}\right)\left(\left.\frac{x^{2}}{2}\right|_{x_{j-1}} ^{x_{j}}-\left.x_{j-1} x\right|_{x_{j-1}} ^{x_{j}}\right)= \\
& =\left(\frac{y_{j}}{h_{j}}-\frac{M_{j} h_{j}}{6}\right)\left(\frac{x_{j}^{2}}{2}-\frac{x_{j-1}^{2}}{2}-x_{j-1} x_{j}+x_{j-1}^{2}\right)= \\
& =\left(\frac{y_{j}}{h_{j}}-\frac{M_{j} h_{j}}{6}\right)\left(\frac{x_{j}^{2}-2 x_{j-1} x_{j}+x_{j-1}^{2}}{2}\right)=\left(\frac{y_{j}}{h_{j}}-\frac{M_{j} h_{j}}{6}\right) \frac{\left(x_{j}-x_{j-1}\right)^{2}}{2}= \\
& =\left(\frac{y_{j}}{h_{j}}-\frac{M_{j} h_{j}}{6}\right) \frac{h_{j}^{2}}{2}=\frac{y_{j} h_{j}}{2}-\frac{M_{j} h_{j}^{3}}{12}
\end{aligned}
$$

Therefore

$$
\begin{aligned}
\int_{x_{j-1}}^{x_{j}} S_{j}(x) d x & =\frac{M_{j-1} h_{j}^{3}}{24}+\frac{M_{j} h_{j}^{3}}{24}+\frac{y_{j-1} h_{j}}{2}-\frac{M_{j-1} h_{j}^{3}}{12}+\frac{y_{j} h_{j}}{2}-\frac{M_{j} h_{j}^{3}}{12}= \\
& =\frac{y_{j-1}+y_{j}}{2} h_{j}-\frac{M_{j-1}+M_{j}}{24} h_{j}^{3}
\end{aligned}
$$




\section{Appendix C. Subroutine for calculating Gauss-Legendre abscissas and weights.}

Given lower and upper limits of integration $\mathrm{x} 1$ and $\mathrm{x} 2$, the routine calculates arrays $\mathrm{x}$ and $\mathrm{w}$ of length $\mathrm{N}$ containing the abscissas and weights of the Gauss-Legendre N-point quadrature formula.

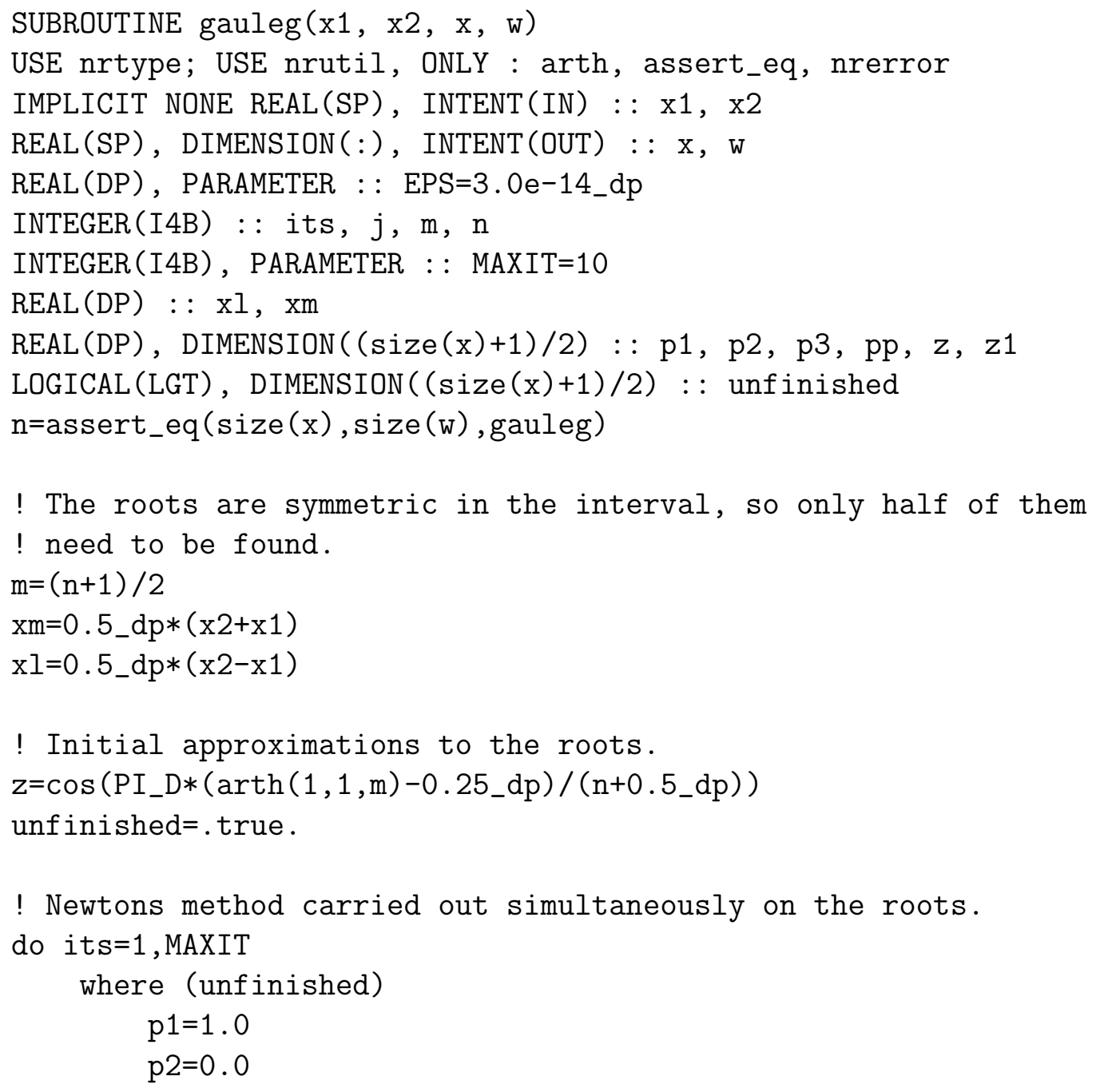


end where

! Loop up the recurrence relation to get the Legendre polynomials

! evaluated at $z$.

do $j=1, n$

where (unfinished)

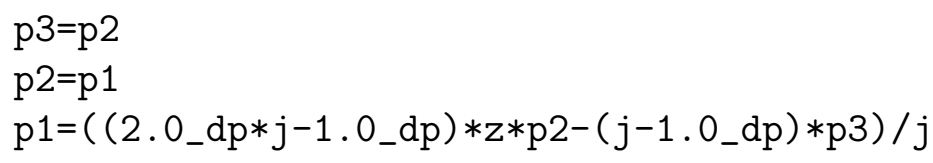

end where

end do

! p1 now contains the desired Legendre polynomials.

! Next pp, the derivatives, are computed by a standard

! relation involving also p2, the polynomials of one lower order. where (unfinished)

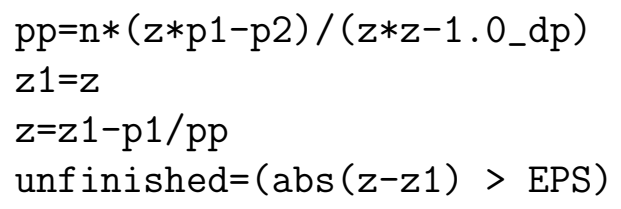

! Scale the root to the desired interval, and put in its

! symmetric counterpart.

$\mathrm{x}(1: \mathrm{m})=\mathrm{xm}-\mathrm{xl} * \mathrm{z}$

$\mathrm{x}(\mathrm{n}: \mathrm{n}-\mathrm{m}+1:-1)=\mathrm{xm}+\mathrm{xl} * \mathrm{z}$

! Compute the weight $\mathrm{w}(\mathrm{n}: \mathrm{n}-\mathrm{m}+1:-1)=\mathrm{w}(1: \mathrm{m})$ and its symmetric

! counterpart.

$\mathrm{w}(1: \mathrm{m})=2.0 \_\mathrm{dp} * \mathrm{xl} /\left(\left(1.0 \_\mathrm{dp}-\mathrm{z} * * 2\right) * \mathrm{pp} * * 2\right)$

$\mathrm{w}(\mathrm{n}: \mathrm{n}-\mathrm{m}+1:-1)={ }_{\mathrm{W}}(1: \mathrm{m})$

END SUBROUTINE gauleg 


\section{Appendix D. Equations for in-plane tension and bending moment.}

Analytically solving the integral from equation 2.17 one obtains the formula that describes the tension in the region of elastic deformations:

$$
\begin{aligned}
T^{e} & =\int_{a}^{z_{1}} \frac{E}{1-\nu^{2}}\left(\frac{z-a}{\rho}\right) d z=\frac{E}{\rho\left(1-\nu^{2}\right)} \int_{a}^{a+b_{1}}(z-a) d z= \\
& =\frac{E}{\rho\left(1-\nu^{2}\right)}\left(\left.\frac{z^{2}}{2}\right|_{a} ^{a+b_{1}}-\left.a z\right|_{a} ^{a+b_{1}}\right)= \\
& =\frac{E}{\rho\left(1-\nu^{2}\right)}\left(\frac{\left(a+b_{1}\right)^{2}}{2}-\frac{a^{2}}{2}-a\left(a+b_{1}\right)+a^{2}\right)= \\
& =\frac{E}{\rho\left(1-\nu^{2}\right)}\left(\frac{a^{2}+2 a b_{1}+b_{1}^{2}-a^{2}}{2}-a^{2}-a b_{1}+a^{2}\right)=\frac{E}{\rho\left(1-\nu^{2}\right)} \frac{b_{1}^{2}}{2}
\end{aligned}
$$

using equation 2.7 for $b_{1}$ :

$$
T^{e}=\frac{E}{2 \rho\left(1-\nu^{2}\right)}\left(\frac{C_{1} \sigma_{u n} \rho}{E}\right)^{2}=\frac{C_{1}^{2} \sigma_{u n}^{2} \rho}{2\left(1-\nu^{2}\right) E}
$$

The tension caused by the plastic stresses can be derived by solving equation 2.18:

$$
T^{p}=\int_{z_{1}}^{\frac{t}{2}} C^{\prime}\left(\frac{z-a}{\rho}-\frac{C_{1} \sigma_{u n}}{E}+\varepsilon_{0}\right)^{n} d z=\int_{a+b_{1}}^{\frac{t}{2}} C^{\prime}\left(\frac{z-a}{\rho}-\frac{C_{1} \sigma_{u n}}{E}+\varepsilon_{0}\right)^{n} d z
$$

Let $u$ be a new integration variable, then:

$u=\frac{z-a}{\rho}-\frac{C_{1} \sigma_{u n}}{E}+\varepsilon_{0} \Rightarrow \frac{z-a}{\rho}=u+\frac{C_{1} \sigma_{u n}}{E}-\varepsilon_{0} \Rightarrow z=u \rho+\frac{C_{1} \sigma_{u n} \rho}{E}-\varepsilon_{0} \rho+a$

and

$$
d z=\rho d u
$$

lower integration limit

$$
u_{1}=\frac{a+b_{1}-a}{\rho}-\frac{C_{1} \sigma_{u n}}{E}+\varepsilon_{0}=\frac{b_{1}}{\rho}-\frac{C_{1} \sigma_{u n}}{E}+\varepsilon_{0}=\varepsilon_{0}
$$


upper integration limit

$$
u_{2}=\frac{\frac{t}{2}-a}{\rho}-\frac{C_{1} \sigma_{u n}}{E}+\varepsilon_{0}
$$

Hence, the tension $T^{p}$ becomes:

$$
\begin{aligned}
T^{p} & =\int_{u_{1}}^{u_{2}} C^{\prime} u^{n} \rho d u=\left.C^{\prime} \rho \frac{u^{n+1}}{n+1}\right|_{u_{1}} ^{u_{2}}= \\
& =\frac{C^{\prime} \rho}{n+1}\left(\left(\frac{\frac{t}{2}-a}{\rho}-\frac{C_{1} \sigma_{u n}}{E}+\varepsilon_{0}\right)^{n+1}-\varepsilon_{0}^{n+1}\right)
\end{aligned}
$$

The compressive force caused by the elastic stresses is found by solving equation 2.19:

$$
\begin{aligned}
C^{e} & =\int_{z_{2}}^{a} \frac{E}{1-\nu^{2}}\left(\frac{z-a}{\rho}\right) d z=\frac{E}{\rho\left(1-\nu^{2}\right)} \int_{a-b_{2}}^{a}(z-a) d z= \\
& =\frac{E}{\rho\left(1-\nu^{2}\right)}\left(\left.\frac{z^{2}}{2}\right|_{a-b_{2}} ^{a}-\left.a z\right|_{a-b_{2}} ^{a}\right)= \\
& =\frac{E}{\rho\left(1-\nu^{2}\right)}\left(\frac{a^{2}}{2}-\frac{\left(a-b_{2}\right)^{2}}{2}-a^{2}+a\left(a-b_{2}\right)\right)= \\
& =\frac{E}{\rho\left(1-\nu^{2}\right)}\left(\frac{a^{2}-a^{2}+2 a b_{2}-b_{2}^{2}}{2}-a^{2}+a^{2}-a b_{2}\right)=-\frac{E}{\rho\left(1-\nu^{2}\right)} \frac{b_{2}^{2}}{2}
\end{aligned}
$$

using equation 2.8 for $b_{2}$ :

$$
C_{e}=-\frac{E}{2 \rho\left(1-\nu^{2}\right)}\left(\frac{C_{1} \sigma_{u n} \rho}{E}\right)^{2}=-\frac{C_{1}^{2} \sigma_{u n}^{2} \rho}{2\left(1-\nu^{2}\right) E}
$$

Solving equation 2.20 gives the expression for the compressive force caused by the plastic stresses:

$$
C^{p}=-\int_{-\frac{t}{2}}^{z_{2}} C^{\prime}\left|\frac{z-a}{\rho}+\frac{C_{1} \sigma_{u n}}{E}-\varepsilon_{0}\right|^{n} d z=-\int_{-\frac{t}{2}}^{a-b_{2}} C^{\prime}\left|\frac{z-a}{\rho}+\frac{C_{1} \sigma_{u n}}{E}-\varepsilon_{0}\right|^{n} d z
$$

Let $u$ be a new integration variable, then:

$u=\frac{z-a}{\rho}+\frac{C_{1} \sigma_{u n}}{E}-\varepsilon_{0} \Rightarrow \frac{z-a}{\rho}=u-\frac{C_{1} \sigma_{u n}}{E}+\varepsilon_{0} \quad \Rightarrow \quad z=u \rho-\frac{C_{1} \sigma_{u n} \rho}{E}+\varepsilon_{0} \rho+a$ and

$$
d z=\rho d u
$$

lower integration limit

$$
u_{1}=\frac{-\frac{t}{2}-a}{\rho}+\frac{C_{1} \sigma_{u n}}{E}-\varepsilon_{0}
$$

upper integration limit

$$
u_{2}=\frac{a-b_{2}-a}{\rho}+\frac{C_{1} \sigma_{u n}}{E}-\varepsilon_{0}=-\frac{b_{2}}{\rho}+\frac{C_{1} \sigma_{u n}}{E}-\varepsilon_{0}=-\varepsilon_{0}
$$


Hence, the compressive force $C^{p}$ becomes:

$$
\begin{aligned}
C^{p} & =-\int_{u_{1}}^{u_{2}} C^{\prime}|u|^{n} \rho d u=-\left.C^{\prime} \rho \frac{|u|^{n+1}}{n+1}\right|_{u_{1}} ^{u_{2}}= \\
& =-\frac{C^{\prime} \rho}{n+1}\left|\varepsilon_{0}^{n+1}-\right| \frac{-\frac{t}{2}-a}{\rho}+\frac{C_{1} \sigma_{u n}}{E}-\left.\varepsilon_{0}\right|^{n+1} \mid
\end{aligned}
$$

Closed form solution of equations 2.26 and 2.27 gives expressions for the parts of the total bending moment in the tension region.

$$
\begin{aligned}
M_{T}^{e} & =\int_{a}^{z_{1}} \frac{E}{1-\nu^{2}}\left(\frac{z-a}{\rho}\right) z d z=\frac{E}{\rho\left(1-\nu^{2}\right)} \int_{a}^{a+b_{1}}\left(z^{2}-a z\right) d z= \\
& =\frac{E}{\rho\left(1-\nu^{2}\right)}\left(\left.\frac{z^{3}}{3}\right|_{a} ^{a+b_{1}}-\left.\frac{a z^{2}}{2}\right|_{a} ^{a+b_{1}}\right)= \\
& =\frac{E}{\rho\left(1-\nu^{2}\right)}\left(\frac{\left(a+b_{1}\right)^{3}}{3}-\frac{a^{3}}{3}-\left(\frac{a\left(a+b_{1}\right)^{2}}{2}-\frac{a^{3}}{2}\right)\right)= \\
& =\frac{E}{\rho\left(1-\nu^{2}\right)}\left(\frac{a^{3}+3 a^{2} b_{1}+3 a b_{1}^{2}+b_{1}^{3}-a^{3}}{3}-\left(\frac{a^{3}+2 a^{2} b_{1}+a b_{1}^{2}-a^{3}}{2}\right)\right)= \\
& =\frac{E}{\rho\left(1-\nu^{2}\right)}\left(\frac{3 a^{2} b_{1}+3 a b_{1}^{2}+b_{1}^{3}}{3}-\frac{2 a^{2} b_{1}+a b_{1}^{2}}{2}\right)= \\
& =\frac{E}{\rho\left(1-\nu^{2}\right)}\left(\frac{6 a^{2} b_{1}+6 a b_{1}^{2}+2 b_{1}^{3}-6 a^{2} b_{1}-3 a b_{1}^{2}}{6}\right)=\frac{E}{\rho\left(1-\nu^{2}\right)}\left(\frac{2 b_{1}^{3}+3 a b_{1}^{2}}{6}\right)= \\
& =\frac{E}{\rho\left(1-\nu^{2}\right)} b_{1}^{2}\left(\frac{b_{1}}{3}+\frac{a}{2}\right)=\frac{E\left(1-\nu^{2}\right)}{\rho\left(\frac{C_{1} \sigma_{u n} \rho}{E}\right)^{2}\left(\frac{C_{1} \sigma_{u n} \rho}{3 E}+\frac{a}{2}\right) \Rightarrow} \Rightarrow \\
M_{T}^{p} & =\int_{z_{1}}^{\frac{t}{2}} C^{\prime}\left(\frac{z-a}{\rho}-\frac{C_{1} \sigma_{u n}}{E}+\varepsilon_{0}\right)^{n} z d z=\int_{a+b_{1}}^{\frac{t}{2}} C^{\prime}\left(\frac{z-a}{\rho}-\frac{C_{1} \sigma_{u n}}{E}+\varepsilon_{0}\right)^{n} z d z
\end{aligned}
$$

Let $u$ be a new integration variable, then:

$u=\frac{z-a}{\rho}-\frac{C_{1} \sigma_{u n}}{E}+\varepsilon_{0} \Rightarrow \frac{z-a}{\rho}=u+\frac{C_{1} \sigma_{u n}}{E}-\varepsilon_{0} \Rightarrow z=u \rho+\frac{C_{1} \sigma_{u n} \rho}{E}-\varepsilon_{0} \rho+a$

and

$$
d z=\rho d u
$$

lower integration limit

$$
u_{1}=\frac{a+b_{1}-a}{\rho}-\frac{C_{1} \sigma_{u n}}{E}+\varepsilon_{0}=\frac{b_{1}}{\rho}-\frac{C_{1} \sigma_{u n}}{E}+\varepsilon_{0}=\varepsilon_{0}
$$

upper integration limit

$$
u_{2}=\frac{\frac{t}{2}-a}{\rho}-\frac{C_{1} \sigma_{u n}}{E}+\varepsilon_{0}
$$


Hence, the moment $M_{T}^{p}$ becomes:

$$
\begin{aligned}
M_{T}^{p} & =\int_{u_{1}}^{u_{2}} C^{\prime} u^{n}\left(u \rho+\frac{C_{1} \sigma_{u n} \rho}{E}-\varepsilon_{0} \rho+a\right) \rho d u= \\
& =C^{\prime} \rho\left(\int_{u_{1}}^{u_{2}} \rho u^{n+1} d u+\int_{u_{1}}^{u_{2}}\left(\frac{C_{1} \sigma_{u n} \rho}{E}-\varepsilon_{0} \rho+a\right) u^{n} d u\right)= \\
& =C^{\prime} \rho\left(\left.\frac{\rho}{n+2} u^{n+2}\right|_{u_{1}} ^{u_{2}}+\left.\left(\frac{C_{1} \sigma_{u n} \rho}{E}-\varepsilon_{0} \rho+a\right) \frac{u^{n+1}}{n+1}\right|_{u_{1}} ^{u_{2}}\right)= \\
& =C^{\prime} \rho\left(\frac{\rho}{n+2}\left(\left(\frac{\frac{t}{2}-a}{\rho}-\frac{C_{1} \sigma_{u n}}{E}+\varepsilon_{0}\right)^{n+2}-\varepsilon_{0}^{n+2}\right)+\left(\frac{C_{1} \sigma_{u n} \rho}{E}-\varepsilon_{0} \rho+a\right) \times\right. \\
& \left.\times \frac{1}{n+1}\left(\left(\frac{\frac{t}{2}-a}{\rho}-\frac{C_{1} \sigma_{u n}}{E}+\varepsilon_{0}\right)^{n+1}-\varepsilon_{0}^{n+1}\right)\right) \Rightarrow \\
M_{T}^{p} & =C^{\prime} \rho\left(\frac{\rho}{n+2}\left(\left(\frac{\frac{t}{2}-a}{\rho}-\frac{C_{1} \sigma_{u n}}{E}+\varepsilon_{0}\right)^{n+2}-\varepsilon_{0}^{n+2}\right)+\left(\frac{C_{1} \sigma_{u n} \rho}{E}-\varepsilon_{0} \rho+a\right) \times\right. \\
& \left.\times \frac{1}{n+1}\left(\left(\frac{\frac{t}{2}-a}{\rho}-\frac{C_{1} \sigma_{u n}}{E}+\varepsilon_{0}\right)^{n+1}-\varepsilon_{0}^{n+1}\right)\right)
\end{aligned}
$$

Solving analytically equations 2.28 and 2.29 one obtains expressions for the parts of the total bending moment in the compression region:

$$
\begin{aligned}
M_{C}^{e} & =\int_{z_{2}}^{a} \frac{E}{1-\nu^{2}}\left(\frac{z-a}{\rho}\right) z d z=\frac{E}{\rho\left(1-\nu^{2}\right)} \int_{a-b_{2}}^{a}\left(z^{2}-a z\right) d z= \\
& =\frac{E}{\rho\left(1-\nu^{2}\right)}\left(\left.\frac{z^{3}}{3}\right|_{a-b_{2}} ^{a}-\left.\frac{a z^{2}}{2}\right|_{a-b_{2}} ^{a}\right)= \\
& =\frac{E}{\rho\left(1-\nu^{2}\right)}\left(\frac{a^{3}}{3}-\frac{\left(a-b_{2}\right)^{3}}{3}-\left(\frac{a^{3}}{2}-\frac{a\left(a-b_{2}\right)^{2}}{2}\right)\right)= \\
& =\frac{E}{\rho\left(1-\nu^{2}\right)}\left(\frac{a^{3}-a^{3}+3 a^{2} b_{2}-3 a b_{2}^{2}+b_{2}^{3}}{3}-\left(\frac{\left.a^{3}-a^{3}+2 a^{2} b_{2}-a b_{2}^{2}\right)}{2}\right)\right)= \\
& =\frac{E}{\rho\left(1-\nu^{2}\right)}\left(\frac{3 a^{2} b_{2}-3 a b_{2}^{2}+b_{2}^{3}}{3}-\frac{2 a^{2} b_{2}-a b_{2}^{2}}{2}\right)= \\
& =\frac{E}{\rho\left(1-\nu^{2}\right)}\left(\frac{6 a^{2} b_{2}-6 a b_{2}^{2}+2 b_{2}^{3}-6 a^{2} b_{2}+3 a b_{2}^{2}}{6}\right)=\frac{E}{\rho\left(1-\nu^{2}\right)}\left(\frac{2 b_{2}^{3}-3 a b_{2}^{2}}{6}\right)= \\
& =\frac{E}{\rho\left(1-\nu^{2}\right)} b_{2}^{2}\left(\frac{b_{2}}{3}-\frac{a}{2}\right)=\frac{E}{\rho\left(1-\nu^{2}\right)}\left(\frac{C_{1} \sigma_{u n} \rho}{E}\right)^{2}\left(\frac{C_{1} \sigma_{u n} \rho}{3 E}-\frac{a}{2}\right) \Rightarrow \\
M_{C}^{p} & =-\int_{-\frac{t}{2}}^{z_{2}} C^{\prime}\left|\frac{z-a}{\rho}+\frac{C_{1} \sigma_{u n}}{E}-\varepsilon_{0}\right|^{n} z d z=-\int_{-\frac{t}{2}}^{a-b_{2}} C^{\prime}\left|\frac{z-a}{\rho}+\frac{C_{1} \sigma_{u n}}{E}-\varepsilon_{0}\right|^{n} z d z \\
M_{C}^{e}=\frac{C_{1}^{2} \sigma_{u n}^{2} \rho}{E\left(1-\nu^{2}\right)}\left(\frac{C_{1} \sigma_{u n} \rho}{3 E}-\frac{a}{2}\right) & (\mathrm{D}-7)
\end{aligned}
$$


Let $u$ be a new integration variable, then:

$u=\frac{z-a}{\rho}+\frac{C_{1} \sigma_{u n}}{E}-\varepsilon_{0} \Rightarrow \frac{z-a}{\rho}=u-\frac{C_{1} \sigma_{u n}}{E}+\varepsilon_{0} \Rightarrow z=u \rho-\frac{C_{1} \sigma_{u n} \rho}{E}+\varepsilon_{0} \rho+a$

and

$$
d z=\rho d u
$$

lower integration limit

$$
u_{1}=\frac{-\frac{t}{2}-a}{\rho}+\frac{C_{1} \sigma_{u n}}{E}-\varepsilon_{0}
$$

upper integration limit

$$
u_{2}=\frac{a-b_{2}-a}{\rho}+\frac{C_{1} \sigma_{u n}}{E}-\varepsilon_{0}=\frac{-b_{2}}{\rho}+\frac{C_{1} \sigma_{u n}}{E}-\varepsilon_{0}=-\varepsilon_{0}
$$

Hence, the moment $M_{C_{2}}^{p}$ becomes:

$$
\begin{aligned}
M_{C}^{p} & =-\int_{u_{1}}^{u_{2}} C^{\prime}|u|^{n}\left(u \rho-\frac{C_{1} \sigma_{u n} \rho}{E}+\varepsilon_{0} \rho+a\right) \rho d u= \\
& =-C^{\prime} \rho\left(\int_{u_{1}}^{u_{2}} \rho|u|^{n+1} d u+\int_{u_{1}}^{u_{2}}\left(\varepsilon_{0} \rho+a-\frac{C_{1} \sigma_{u n} \rho}{E}\right)|u|^{n} d u=\right. \\
& =-C^{\prime} \rho\left(\left.\frac{\rho}{n+2}|u|^{n+2}\right|_{u_{1}} ^{u_{2}}+\left.\left(\varepsilon_{0} \rho+a-\frac{C_{1} \sigma_{u n} \rho}{E}\right) \frac{1}{n+1}|u|^{n+1}\right|_{u_{1}} ^{u_{2}}\right)= \\
& =C^{\prime} \rho \mid \frac{\rho}{n+2}\left(\varepsilon_{0}^{n+2}-\left|\frac{-\frac{t}{2}-a}{\rho}+\frac{C_{1} \sigma_{u n}}{E}-\varepsilon_{0}\right|^{n+2}\right)-\left(\varepsilon_{0} \rho+a-\frac{C_{1} \sigma_{u n} \rho}{E)}\right) \frac{1}{n+1} \times \\
& \times\left(\varepsilon_{0}^{n+1}-\left|\frac{-\frac{t}{2}-a}{\rho}+\frac{C_{1} \sigma_{u n}}{E}-\varepsilon_{0}\right|^{n+1}\right) \mid \Rightarrow \\
M_{C}^{p} & =C^{\prime} \rho \mid \frac{\rho}{n+2}\left(\varepsilon_{0}^{n+2}-\left|\frac{-\frac{t}{2}-a}{\rho}+\frac{C_{1} \sigma_{u n}}{E}-\varepsilon_{0}\right|^{n+2}\right)-\left(\varepsilon_{0} \rho+a-\frac{C_{1} \sigma_{u n} \rho}{E)}\right) \frac{1}{n+1} \times \\
& \times\left(\varepsilon_{0}^{n+1}-\left|\frac{-\frac{t}{2}-a}{\rho}+\frac{C_{1} \sigma_{u n}}{E}-\varepsilon_{0}\right|^{n+1}\right) \mid
\end{aligned}
$$





\section{Bibliography}

[1] R. D. Cook, D. S. Malkus, M. E. Plesha, and R. J. Witt. Concepts and Applications of Finite Element Analysis. John Wiley and Sons, Inc., 4th edition, 2002.

[2] S. Rajendran and K. M. Liew. Optimal stress sampling points of plane triangular elements for patch recovery of nodal stresses. International Journal for Numerical Methods in Engineering, 58(4):579-607, 2003.

[3] C. J. Burgoyne and M. A. Crisfield. Numerical integration strategy for plates and shells. International Journal for Numerical Methods in Engineering, 29(1):105-121, 1990 .

[4] T. Belytschko, W. K. Liu, and B. Moran. Nonlinear Finite Elements for Continua and Structures. John Wiley and Sons, Ltd., 2000.

[5] R. H. Wagoner and M. Li. Advances in springback. In L.M. Smith, F. Pourboghrat, J.W. Yoon, and T.B. Stoughton, editors, NUMISHEET 2005. Proceedings of the 6th international conference and workshop on numerical simulation of $3 D$ sheet metal forming processes, volume A, pages 209-214, Detroit, MI, U.S.A., 2005.

[6] J. C. Simo and J. G. Kennedy. On a stress resultant geometrically exact shell model. part v. nonlinear plasticity: formulation and integration algorithms. Computer Methods in Applied Mechanics and Engineering, 96(2):133-171, 1992.

[7] H. Armen, A. Pifko, and H.S. Levine. Finite element analysis of structures in the plastic range. Report NASA CR-1649, Grumman Aerospace Corporation, 1971.

[8] K. E. Atkinson. An introduction to numerical analysis. John Wiley and Sons, Inc., 2nd edition, 1989.

[9] P.J. Davis and P. Rabinowitz. Methods of numerical integration. Academic Press, Inc., 2nd edition, 1985.

[10] J.H. Ahlberg, E.N. Nilson, and J.L. Walsh. The Theory of Splines and Their Applications. NY, Academic Press, 1967.

[11] G.H. Golub and Welsch J.H. Calculation of gauss quadrature rules. Report N-CS 81, Stanford University, november 1967. 
[12] W.H. Press, S.A. Teukolsky, W.T. Vetterling, and B.P. Flannery. Numerical Recipes in Fortran 77. The Art of Scientific Computing. Press Syndicate of the University of Cambridge, 2nd edition, 1992.

[13] W.H. Press, S.A. Teukolsky, W.T. Vetterling, and B.P. Flannery. Numerical Recipes in Fortran 90. Press Syndicate of the University of Cambridge, 2nd edition, 1996.

[14] G.H. Golub. Some modified egenvalue problems. Report Stan-CS-234-71, Stanford University, august 1971.

[15] J. Burkardt. Quadpack - a library of fortran90 routines for estimating integrals. http://www.csit.fsu.edu/ burkardt/f_src/quadpack/quadpack.html, FSU - School of Computational Science, 2006.

[16] R.H. Wagoner. Fundamental aspects of springback in sheet metal forming. In Huh H. Yang D.-Y., Oh S.I. and Kim Y.H., editors, NUMISHEET 2002, The Fifth International Conference and Workshop on Numerical Simulation of $3 D$ Sheet Forming Processes., pages 13 - 24, Jeju Island, Korea, 2002.

[17] R. J. Rice. A metalgorithm for adaptive quadrature. Journal of the ACM (JACM), 22(1):61-82, 1975.

[18] W. Gander and W. Gautschi. Adaptive quadrature - revisited. BIT Numerical Mathematics, 40(1):84-101, 2000.

[19] T. O. Espelid. Doubly adaptive quadrature routines based on newton-cotes rules. BIT Numerical Mathematics, 43(2):319-337, 2003.

[20] S. Garribba, L. Quartapelle, and G. Reina. Algorithm 36 sniff: Efficient self-tuning algorithm for numerical integration. Computing, 20(4):363-375, 1978.

[21] J. Berntsen, T. O. Espelid, and T. Sorevik. On the subdivision strategy in adaptive quadrature algorithms. Journal of Computational and Applied Mathematics, 35(13):119-132, 1991.

[22] H.D. Shapiro. Increasing robustness in global adaptive quadrature through interval selection heuristics. ACM Trans. Math. Softw., 10(2):117-139, 1984.

[23] P.E. Hennion. Algorithm 77: interpolation, differentiation and integration. Communications of the ACM, 1962.

[24] T. Meinders. Plate and shell theory. Report, University of Twente, 2004.

[25] C.K. Lee and R.E. Hobbs. Automatic adaptive refinement for plate bending problems using reissner-mindlin plate bending elements. International Journal for Numerical Methods in Engineering, 41(1):1-63, 1998.

[26] B. K. Chun. Study on hardening models and numerical implementation for springback prediction. PhD thesis, The Ohio State University, 2001. 
[27] M. Avetisyan, T. Meinders, and J. Huetink. Influence of an accurate trimming operation on springback. International Journal of Forming Processes, special issue:31-51, 2005.

[28] M. Avetisyan. Numerical trimming operation with respect to springback. Report P05.1.014, NIMR-University of Twente, 2004.

[29] I.A. Burchitz. Influence of discretisation error on springback prediction. Report, NIMR-University of Twente, 2006. 